\title{
Engineering Ferroelectric Switching Dynamics
}

Ohirban Ghosh

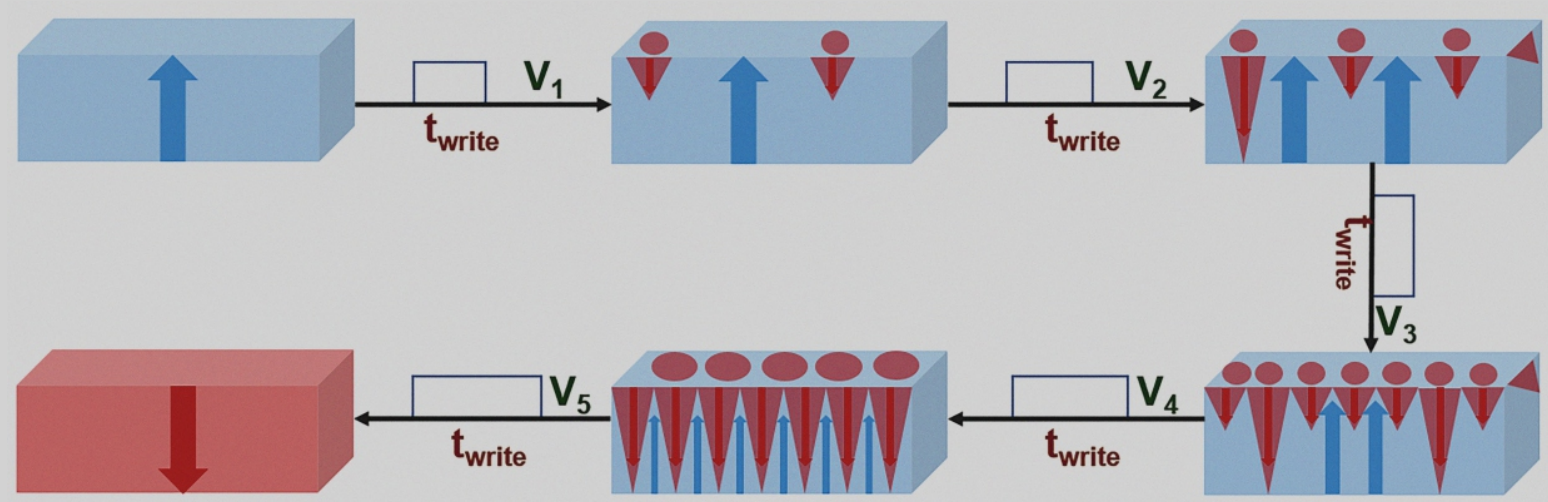


ENGINEERING FERROELECTRIC

SWITCHING DYNAMICS

By

Anirban Ghosh 


\section{Ph.D. Committee}

Chairman \& Secretary: Prof. dr. ir. J.W.M. Hilgenkamp

PhD Supervisor: $\quad$ Prof. dr. ing. A.J.H.M. Rijnders

Co-Supervisor: Prof. dr. ir. G. Koster

Committee Members:

Prof. Marin Alexe

Prof. ir. Beatrice Noheda

Prof. dr. ir. W. G. van der Wiel

Prof. dr. ir. H. J.W. Zandvliet

\section{Referee}

Dr.ir. R.J.E. Hueting

\author{
University of Warwick \\ University of Groningen \\ University of Twente \\ University of Twente
}

University of Twente

\section{Cover}

The cover illustrates the interface nucleation controlled switching in a ferroel ectric thin film and how by controlling the distribution of nucleation energy one can achieve a multiple states instead of thermodynamics limited bi-stable switching. The difference between a double well potential for a bi-stable system and multiple energy states for a multistate system is schematically illustrated (energies not drawn to scale).

The research described in this thesis was performed with the Inorganic Materials Science group and the MESA+ Institute for Nanotechnology at the University of Twente, Enschede, The Netherlands. This work was supported by NanoNextNL, a micro and nanotechnology consortium of the Government of the Netherlands and 130 partners.

Anirban Ghosh: Ferroelectric switching dynamics

PhD Thesis, Enschede, The Netherlands,

ISBN: 978-90-365-4196-1

DOI: $10.3990 / 1.9789036541961$

Printed By: Gildeprint, Enschede

Copyright ( A. Ghosh, 2016 


\title{
ENGINEERING FERROELECTRIC SWITCHING DYNAMICS
}

\section{DISSERTATION}

\author{
to obtain
}

the degree of Doctor at the University of Twente, on the authority of the rector magnificus,

Prof. dr. H. Brinksma,

on account of the decision of the graduation committee,

to be publicly defended

on Friday, 23rd September 2016, at 12:45

by

Anirban Ghosh

born on $7^{\text {th }}$ December 1984

in Asansol, Burdwan, India 
This thesis has been approved by

Prof. dr. ing. A.J.H.M. Rijnders (promotor)

and Prof. dr. ir. G. Koster (co-promotor) 


\section{Table of Contents}

1 Engineering Ferroelectric Switching Dynamics 1

$\begin{array}{ll}1.1 \text { Motivation } & 2\end{array}$

1.2 Outline of the thesis $\quad 4$

$\begin{array}{ll}1.3 \text { References } & 6\end{array}$

2 Fabrication and Structural Characterization of PZT-ZnO Heterostructures 9

$\begin{array}{ll}2.1 \text { Introduction } & 10\end{array}$

$\begin{array}{ll}2.2 \text { Results and Discussion } & 12\end{array}$

$\begin{array}{ll}\text { 2.2.1 Growth } & 12\end{array}$

2.2.2 Structural Characterizations 13

$\begin{array}{ll}2.3 \text { Conclusions } & 17\end{array}$

$\begin{array}{ll}2.4 \text { References } & 19\end{array}$

3 Tunable And Temporally Stable Ferroelectric Imprint Through Polarization $\begin{array}{ll}\text { Coupling } & 23\end{array}$

$\begin{array}{ll}3.1 \text { Introduction } & 24\end{array}$

3.2 Results and Discussion $\quad 26$

$\begin{array}{ll}\text { 3.2.1 Theory } & 26\end{array}$

$\begin{array}{ll}\text { 3.2.2 Experiment } & 27\end{array}$

$\begin{array}{ll}3.3 \text { Conclusions } & 32\end{array}$ 
4 Multi-stability in Bi-stable Ferroelectric Materials Towards Adaptive Applications

4.1 Introduction

4.2 Experimental Section

40

4.3 Results and Discussion

4.4 Conclusions 58

4.5 References 59

5 Electrical Characterizations of PZT-ZnO Heterostructures

5.1 Introduction

5.2 Transport Mechanisms and Current-Voltage (IV) Measurements

5.2.1 Bulk Limited Conduction Mechanism

5.2.2 Interface Limited Conduction

5.3 Capacitance-Voltage Measurements 75

5.3.1 Free Carrier Density 76

5.3.2 Interface Trap Density

5.4 Admittance angle 78

5.5 Experimental Section 78

5.6 Results and Discussion 79

5.6.1 Temperature dependent I-V measurements 79

5.6.1 Temperature dependent $\mathrm{C}-\mathrm{V}$ measurements 85 
$\begin{array}{ll}\text { 5.6.4 Admittance Angle } & 90\end{array}$

$\begin{array}{ll}5.7 \text { Conclusions } & 91\end{array}$

$\begin{array}{ll}5.8 \text { References } & 92\end{array}$

6 Meso-frequency Study of Switching Dynamics in PZT-ZnO Heterostructures 97

$\begin{array}{ll}6.1 \text { Introduction } & 98\end{array}$

$\begin{array}{ll}6.2 \text { Experimental Section } & 104\end{array}$

$\begin{array}{ll}\text { 6.3 Results and Discussion } & 105\end{array}$

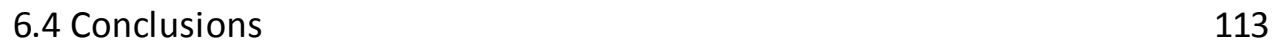

$\begin{array}{ll}6.5 \text { References } & 115\end{array}$

Summary

Samenvating

Acknowledgements 


\section{CHAPTER 1}

\section{ENGINEERING FERROELECTRIC SWITCHING DYNAMICS}

A general introduction to ferroelectric switching dynamics is presented in this chapter. The thesis explores the possibility of manipulating the ferroelectric switching dynamics at the ferroelectric-electrode interface. This chapter broadly introduces how controlling the switching dynamics can help us actualise nonvolatile adaptive switching in ferroelectrics, necessary for mimicking biological synapses in real devices. The chapter concludes with an outline of the thesis. 


\subsection{Motivation}

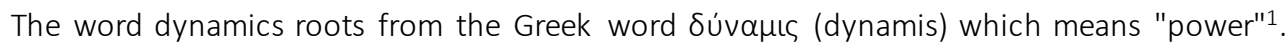
Consequently, this branch of science deals with the effects of forces on motion such as aerodynamics which deals with the motion of gas, and thermodynamics which describes the relationship between thermal and mechanical energy etc. In ferroelectrics, switching dynamics deals with the study of domain wall motion under an applied electric field ${ }^{2-4}$. But in order to understand a physical system fully it is not only important to understand the dynamical behaviour of the system, but also the degrees of freedom of the system which determine the number of independent ways in which a system can move. For an ensemble the degrees of freedom are determined by its stochastic variables. Apropos, within systems at real device scale (present day ferroelectric based devices are $130 \mathrm{~nm}$ in lateral dimension), it is pertinent to treat them as an ensemble rather than as a single entity.

Ferroelectrics, because of their non-volatility and bi-stability and, most importantly, their switchability between the two states using an applied electric field, have found applications such as non-volatile random access memory (NVRAM) devices ${ }^{3,5}$. Ferroelectric RAM (FeRAM) devices were first proposed by Dudley Allen Buck in his master's thesis in 1952 at MIT ${ }^{6}$. It was shown that ferroelectrics can not only be used for storing memory but also for logic operations. Field effect transistors based on ferroelectrics (FeFET), as well as ferroelectric tunnel junctions (FTJ) based devices in recent years have also been realized for the realization of non-destructive read out memories ${ }^{7-12}$.

Even though the crystal structure and internal chemical environment renders the bi-stability in ferroelectrics 13,14 , the actual switching in ferroelectrics takes place through nucleation and growth mechanisms $3,4,15,16$ of ferroelectric domains. In FeRAM and FeFET, the device performance characterised by the switching speed, remnant polarization, coercive voltages, leakage current, reliability and stability, is of paramount importance. For the above devices, since domain wall switching dynamics determines the switching speed, coercive voltages, operating voltages and energy consumption etc., it gave rise to a plethora of research into the domain wall switching dynamics and statistics treating the ferroelectrics as ideal insulators. On the other hand, ferroelectrics were also treated as semiconductors to explain 
their leakage and electrode charge injection induced properties which determine their stability and reliability $3,17,18$. In recent years, with the advent of piezo force microscopy (PFM) switching dynamical studies have also been carried out ${ }^{19,20}$ to understand the domain dynamics at nano dimensions.

Conventionally, the FeRAM and FeFETs were thought to be utilized only for binary applications for Boolean computation, hence the main focus of the research was to minimize the disorder and to achieve a very narrow distribution of switching times or voltages in ferroelectrics. The major reason for a distribution of switching times and voltages was ascribed to either a distribution of domain growth energies or domain nucleation energies $16,21,22$. The distribution of switching times as a tool or as a benison was never quite envisioned, especially due to the inability to control them, due to their dynamic nature rather than the desired static one.

In order to actualize brain inspired adaptive computation based on neural networks, beyond the conventional Boolean based logic, one needs switchable multi-stable non-volatile states $^{23-25}$. In this context, having a ferroelectric with a distribution of switching times or voltages can be imagined as equivalent to a multi-stable adaptive ferroelectric. The ability of a neural network to learn depends on the number of degrees of freedom available to the network (for an electronic device it means the number of different switchable states). The number of degrees of freedom determines the plasticity of the system, i.e., its capability of approximating the training set $23-28$. Increasing the plasticity helps to reduce the training error, decreasing the plasticity excessively can lead to a large training and test error.

The biggest challenge in achieving this lies in realizing these multiple (polarization) states in a controlled, tunable as well as stable manner. In essence in order to bring about multistability and adaptability in a bi-stable ferroelectric we need to have disorder which is stable and static in nature.

With recent advancements in thin film deposition techniques especially for interface engineering, many fascinating and hitherto unknown properties have been unearthed and contrived $^{29,30}$. In ferroelectric thin films, since the nucleation takes place at the ferroelectricelectrode interface, it gives us the opportunity to manipulate the nucleation energy and 
statistics at the ferroelectric-electrode interface which is dependent on the local electric field distribution.

In this thesis, it is shown how one can achieve the desired plasticity/adaptability in a ferroelectric thin film by making the nucleation to be the rate determining stage for switching (nucleation controlled switching) and how to manipulate the order to disorder ratio of the effective field at the ferroelectric-electrode to have a distribution of nucleation times. The main challenge here is, since both the nucleation and growth activation energies of switching are of similar magnitude and because the ferroelectric films mostly switch by sideways movement of the domain walls (domain growth) ${ }^{3}$ it was not possible to switch the ferroelectric through nucleation alone. Nucleation is an interface phenomenon therefore it is possible to control it by engineering the ferroelectric-electrode interface and by making the nucleation energy much higher than the growth energy of the domain wall.

\subsection{Outline of the Thesis}

This thesis studies the switching dynamics in ferroelectric $\mathrm{PbZr}_{x} \mathrm{Ti}_{(1-x)} \mathrm{O}_{3}(\mathrm{PZT})$ thin films and introduces the idea of manipulating it for adaptive applications. Below the chapters addressed in this thesis are briefly described.

In Chapter 2 the growth and structural characterizations is described of the PZT and PZT/ZnO heterostructure thin films which were used to carry out experiments in the later chapters. $\mathrm{PZT} / \mathrm{ZnO}$ heterostructures with four different thicknesses of $\mathrm{ZnO}$ were grown using the pulsed laser deposition technique (PLD) and were studied using X-Ray Diffraction (XRD), Scanning Electron Microscopy (SEM), Transmission Electron Microscopy (TEM) and Atomic Force Microscopy (AFM).

In Chapter 3, a methodology to tune the ferroelectric imprint in a temporally stable manner based on the coupling between the switchable polarization of PZT and non-switchable polarization of $\mathrm{ZnO}$ is introduced. Herein a method to manipulate the local electric field exerted at the ferroelectric-electrode interface is shown. The stability of the imprint to 
electric field stress with time and cycling was also measured. It is also shown that the local electric field at the ferroelectric-electrode interface can be tuned utilizing the $\mathrm{ZnO}$ thickness.

In Chapter 4, it is described how the switching dynamics of the ferroelectric gets modified by the electric field at the ferroelectric-electrode interface. The switching dynamics was studied using the Kolmogorov-Avrami-Ishibashi (KAI) and nucleation limited switching (NLS) model to determine the relevant switching energy scales. In addition, the switching velocities have been analyzed to determine the mechanism of switching. It also shown how the switching statistics can be tuned and how it can be utilized to attain multistability and adaptability in an otherwise bistable ferroelectrics. The significance of these results is discussed in the light of artificial neural networks.

Contrary to previous chapters where only the insulating properties of the ferroelectric PZT are studied, Chapter 5 discusses the semiconducting characteristics of the ferroelectric PZT. The aforementioned heterostructures were treated in terms of MIM (metal insulator metal) and MIS (metal insulator semiconductor) structures. The leakage and interface characteristics were determined using temperature dependent current-voltage and capacitance voltage measurements. The built-in field, free charge concentration, depletion layer thickness, interface trap density etc. was determined. In addition, the ratio of the capacitive to resistive nature, as well as the stability of the capacitive contribution with frequency of these films were determined.

In Chapter 6, different stages of ferroelectric switching using temperature dependent mesofrequency studies are analysed. Different stages of switching were classified in terms of thermally activated and flow motion of domain walls. It was found that a thermal activation free domain wall motion at room temperature can be achieved if the nucleation energy is an order of magnitude higher than the domain growth energy. 


\subsection{References}

1. Wikipedia. (July 2016).

2. D. Jung, M. Dawber, J. Scott, L. Sinnamon and J. Gregg, Integrated Ferroelectrics 48 (1), 59-68 (2002).

3. J. F. Scott, Ferroelectric memories. (Springer Science \& Business Media, 2000).

4. J. F. Scott, L. Kammerdiner, M. Parris, S. Traynor, V. Ottenbacher, A. Shawabkeh and W. F. Oliver, Journal of Applied Physics 64 (2), 787 (1988).

5. C. A. Paz De Araujo, L. D. McMillan, B. M. Melnick, J. D. Cuchiaro and J. F. Scott, Ferroelectrics 104 (1), 241-256 (1990).

6. D. A. B. Ferroelectrics for Digital Information Storage and Switching, Thesis, 1952.

7. S. Mathews, R. Ramesh, T. Venkatesan and J. Benedetto, Science 276 (5310), 238-240 (1997).

8. A. Chanthbouala, V. Garcia, R. O. Cherifi, K. Bouzehouane, S. Fusil, X. Moya, S. Xavier, H. Yamada, C. Deranlot, N. D. Mathur, M. Bibes, A. Barthelemy and J. Grollier, Nature materials 11 (10), 860-864 (2012).

9. J. P. Velev, C.-G. Duan, K. D. Belashchenko, S. S. Jaswal and E. Y. Tsymbal, Physical Review Letters 98 (13) (2007).

10. Z. Wen, C. Li, D. Wu, A. Li and N. Ming, Nature materials 12 (7), 617-621 (2013).

11. J. Hoffman, X. Pan, J. W. Reiner, F. J. Walker, J. P. Han, C. H. Ahn and T. P. Ma, Advanced materials 22 (26-27), 2957-2961 (2010).

12. Y. Watanabe, Applied Physics Letters 66 (14), 1770 (1995).

13. R. E. Cohen, Nature 358 (6382), 136-138 (1992).

14. N. A. Hill, The Journal of Physical Chemistry B 104 (29), 6694-6709 (2000).

15. A. K. Tagantsev and G. Gerra, Journal of Applied Physics 100 (5), 051607 (2006).

16. A. K. Tagantsev, I. Stolichnov, N. Setter, J. S. Cross and M. Tsukada, Physical Review B66 (21), 214109 (2002).

17. L. Pintilie, Charge transport in ferroelectric thin films. (INTECH Open Access Publisher, 2011). 
18. A. K. Tagantsev, I. Stolichnov, E. L. Colla and N. Setter, Journal of Applied Physics 90 (3), 1387 (2001).

19. S. Jesse, B. J. Rodriguez, S. Choudhury, A. P. Baddorf, I. Vrejoiu, D. Hesse, M. Alexe, E. A. Eliseev, A. N. Morozovska, J. Zhang, L. Q. Chen and S. V. Kalinin, Nature materials 7 (3), 209215 (2008).

20. W. Li and M. Alexe, Applied Physics Letters 91 (26), 262903 (2007).

21. Y. A. Genenko, S. Zhukov, S. V. Yampolskii, J. Schütrumpf, R. Dittmer, W. Jo, H. Kungl, M. J. Hoffmann and H. von Seggern, Advanced Functional Materials 22 (10), 2058-2066 (2012). 22. S. Zhukov, Y. A. Genenko and H. von Seggern, Journal of Applied Physics 108(1), 014106 (2010).

23. J. M. Zurada, St. Paul: West,, Introduction to artificial neural systems. (1992.).

24. M. H. Hassoun, Fundamentals of artificial neural networks. (MIT press, 1995).

25. J. Misra and I. Saha, Neurocomputing 74 (1), 239-255 (2010).

26. D. kriesel, A brief introduction to neural networks 2011 (August 15 (2007): .).

27. L. P. Maguire, T. M. McGinnity, B. Glackin, A. Ghani, A. Belatreche and J. Harkin, Neurocomputing 71 (1-3), 13-29 (2007).

28. J. Schmidhuber, Neural networks : the official journal of the International Neural Network Society 61, 85-117 (2015).

29. J. Mannhart and D. Schlom, Science 327 (5973), 1607-1611 (2010).

30. N. Reyren, S. Thiel, A. Caviglia, L. F. Kourkoutis, G. Hammerl, C. Richter, C. Schneider, T. Kopp, A.-S. Rüetschi and D. Jaccard, Science 317 (5842), 1196-1199 (2007). 


\section{FABRICATION AND STRUCTURAL CHARACTERIZATION OF PZT-ZNO HETEROSTRUCTURES}

This chapter describes the sample growth and the structural characterization of the PZT heterostructures used in this thesis. The samples used in this thesis were grown using the pulsed laser deposition (PLD) technique. The structural and morphological characterization carried out using X-ray Diffraction (XRD), Transmission Electron Microscopy (TEM) and Atomic Force Microscopy (AFM) are discussed in this chapter. 


\subsection{Introduction}

Ever since its invention in the late 1950s many potential applications have been envisaged for utilizing the Light Amplification by Stimulated Emission of Radiation (LASER). In materials science one of the most propitious idea was for synthesizing novel material systems at nanoscale ${ }^{1,2}$. Due to their very high energy density (up to $5 \mathrm{~J} / \mathrm{cm}^{2}$ ) and narrow bandwidth (< $1 \mathrm{pm}$ ) and coherency the LASER can be used to vaporize even the most refractory materials with a high level of precision and control. The biggest advantage of depositing materials using a LASER is the very accurate stoichiometry transfer and control as well as very effective control of the morphology by manipulating the deposition conditions ${ }^{2-4}$. Even though the LASER was first used in 1960's for depositing thin films of oxides ${ }^{5}$, the main interest in utilizing the LASER for thin film deposition started in $1980 \mathrm{~s}$ with the discovery of the high $\mathrm{T}_{\mathrm{c}}$ superconductors due to their layered structures ${ }^{6}$. After the realization of reflection highenergy electron diffraction (RHEED) ${ }^{7}$ at high oxygen pressure to monitor thin film growth at atomistic level, PLD was utilized for synthesizing novel artificial materials by designing at atomic scale ${ }^{8-13}$.

In this thesis the PLD technique for growing the epitaxial oxide heterostructures is employed.

\section{Pulsed Laser Deposition}

In Figure 2.1 show a schematic of the PLD thin film growth deposition technique. PLD in principle involves vaporizing small volumes of a target material using small duration $(20-35$ ns) high powered LASER pulses as shown in Figure 2.1. The complete process of pulsed laser deposition comprises of series of steps as mentioned below 2,14:

1. Laser target interaction: At first the high powered laser radiation gets absorbed in a small volume of the target material.

2. Ablation of the target and plasma formation: Due to the high power of the laser high temperature regions form inside a small region of the target leading complete evaporation, ionization of the target material and formation of a plasma. 
3. Deposition of the ablated material: The plasma plume deposits the target material on the heated substrate and this constitutes the transfer of the desired target material.

4. Nucleation and growth on the substrate: The deposited plasma particles nucleate and grow on the surface of the substrate to form a thin film. In case of epitaxial growth the thin film follows the growth direction of the substrate.

\section{Instrumental setup}

In the actual experimental setup the Laser is a KrF excimer Laser with a wavelength of $\lambda=$ $248 \mathrm{~nm}$ with a typical pulse duration of 20-30 ns. The background pressure inside the deposition chamber is kept at $10^{-7}$ mbar. The energy density of the laser can be varied from 1-5 J/cm ${ }^{2}$ and the frequency of the laser repetition can be varied from 1-50 $\mathrm{Hz}$. The laser beam is focused by a lens and projected at an angle of $45^{\circ}$. The laser spot size is of the order of few square millimetres and can be modified by adjusting the lens and mask. The substrate temperature during deposition can be maintained from room temperature up to $950^{\circ} \mathrm{C}$ and the pressure during deposition is kept between $10^{0}$ to $10^{-3}$ mbar using a mass flow controller.

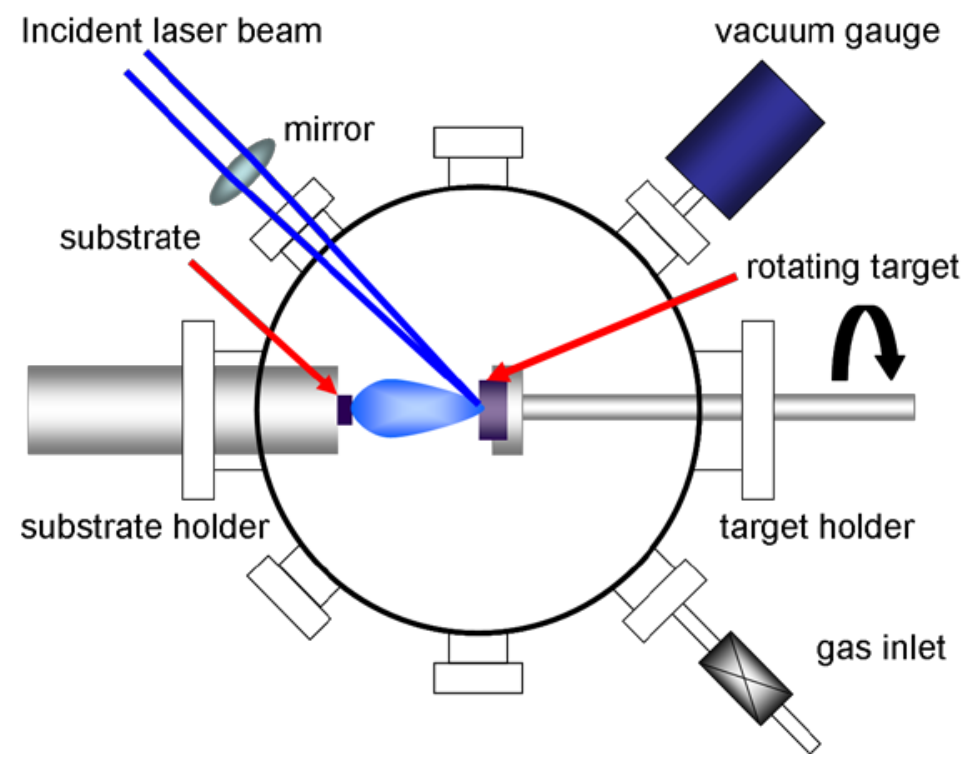

Figure 2. 1 Pulsed Laser Deposition experimental setup 


\section{Patterning}

In order to measure the electrical properties of the heterostructure capacitor structures of $200 \mu \mathrm{m}$ by $200 \mu \mathrm{m}, 100 \mu \mathrm{m}$ by $100 \mu \mathrm{m}$ and $50 \mu \mathrm{m}$ by $50 \mu \mathrm{m}$ were fabricated using pulsed laser deposition followed by photolithography and etching ${ }^{15}$. The process comprises of three processes: a) etching, b) lift-off and c) shadow mask deposition. At first photolithography was used to define the photoresist layer. Dry etching was carried out by Ar ion beam milling at $2.3^{*} 10^{-3} \mathrm{mbar}$ Ar pressure at a rate of $10 \mathrm{~nm} / \mathrm{min}$ to remove the top electrode layer. The electrical resistance between electrodes was measured to make sure the electrodes are disconnected.

\subsection{Results and Discussions}

\section{2. 1 Growth}

Heterostructures of $\mathrm{SrRuO}_{3} \quad(80 \mathrm{~nm}) / \mathrm{PbZr}_{0.58} \mathrm{Ti}_{0.42} \mathrm{O}_{3}(1000 \mathrm{~nm}) \quad(\mathrm{PZT}) / \mathrm{ZnO} \quad(25-150$ $\mathrm{nm}) / \mathrm{SrRuO}_{3}(80 \mathrm{~nm})$ were fabricated on $\mathrm{STiO}_{3}$ (111) substrates using pulsed laser deposition ${ }^{15}$ (Figure 2.2). For the purpose of our experiments, devices with four different thicknesses of $\mathrm{ZnO}, 25 \mathrm{~nm}, 50 \mathrm{~nm}, 100 \mathrm{~nm}$ and $150 \mathrm{~nm}$ as well as a device without $\mathrm{ZnO}$, were fabricated. Hereon, the samples are named PZT25, PZT50, PZT100, PZT150 and PZT0 respectively. The $\mathrm{SrRuO}_{3}$ and $\mathrm{PZT}$ grow epitaxially on $\mathrm{SrTiO}_{3}$ in the (111) direction. $\mathrm{ZnO}$ grows along the (0001) direction on the PZT. In Table 1 the deposition parameters of all materials are summarized. PbZro.58 $\mathrm{Ti}_{0.42} \mathrm{O}_{3}$ is rhombohedral 16,17 and $\mathrm{ZnO}$ has a wurtzite crystal structure ${ }^{18}$. The STO substrates were treated with a dilute buffered HF solution to obtain a single termination ${ }^{19}$ with unit cell step sizes. 
Table 1. Growth conditions for heterostructure growth

\begin{tabular}{|l|l|l|l|}
\hline & SRO & PZT & ZnO \\
\hline $\begin{array}{l}\text { Substrate } \\
\text { temperature }\left({ }^{\circ} \mathrm{C}\right)\end{array}$ & 630 & 600 & 350 \\
\hline $\begin{array}{l}\text { Oxygen pressure } \\
\text { (mbar) }\end{array}$ & 0.13 & 0.11 & 0.05 \\
\hline Laser fluence $\left(\mathrm{J} / \mathrm{cm}^{2}\right)$ & 2.08 & 2.0 & 3.0 \\
\hline Laser frequency $(\mathrm{Hz})$ & 5 & $\begin{array}{l}10^{4} \text { pulses @ 5 Hz } \\
+4 \times 10^{4} \text { pulses @ } \\
10 \mathrm{~Hz}\end{array}$ & 4 \\
\hline
\end{tabular}

\section{2. 2 Structural Characterizations}

\section{X-Ray Diffraction}

The crystallographic properties of the aforementioned heterostructure were investigated by X-Ray diffraction (XRD) (Panalytical X'Pert Powder diffractometer and $X^{\prime}$ Pert MRD) ${ }^{20}$. Figure 2.3 (a) shows the XRD spectrum of device PZT100, indicating the epitaxial (111) growth of PZT and SRO. Figure 2.3 (b) shows the XRD spectrum of the heterostructure around the (321) reflections. The reciprocal space map around (321) and (111) reflections indicate that the PZT is rhombohedral with a lattice constant of $4.08 \AA$. It can be noted from the reciprocal space map that the PZT films are fully relaxed. $\Phi$ scans were performed to determine the inplane epitaxial relationships between the $\mathrm{ZnO}$ films and STO substrates. Figure 2.4 shows that $\mathrm{ZnO}$ films grow along the c-axis perpendicular to the growth plane on the (111) STO substrates. Twelve peaks were observed for the ZnO family, which has six crystal planes with the same angle as the STO in plane and six planes rotated $30^{\circ}$ to the in plane. From the relative position of $\mathrm{ZnO}$ and STO, $\{100\}$ families, the in-plane relationships obtained was $<1$ $100>\mathrm{ZnO} \|<0-11>$ STO and $<11-20>\mathrm{ZnO} \|<0-11>$ STO on the (111) STO substrates $21,22$. 


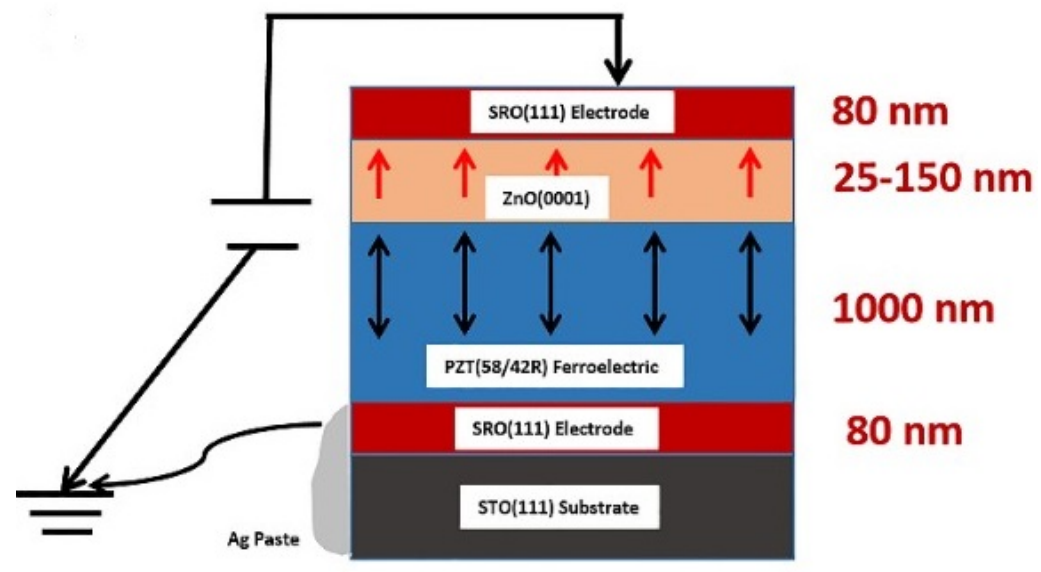

Figure 2.2 Schematic of PZT-ZnO heterostructures

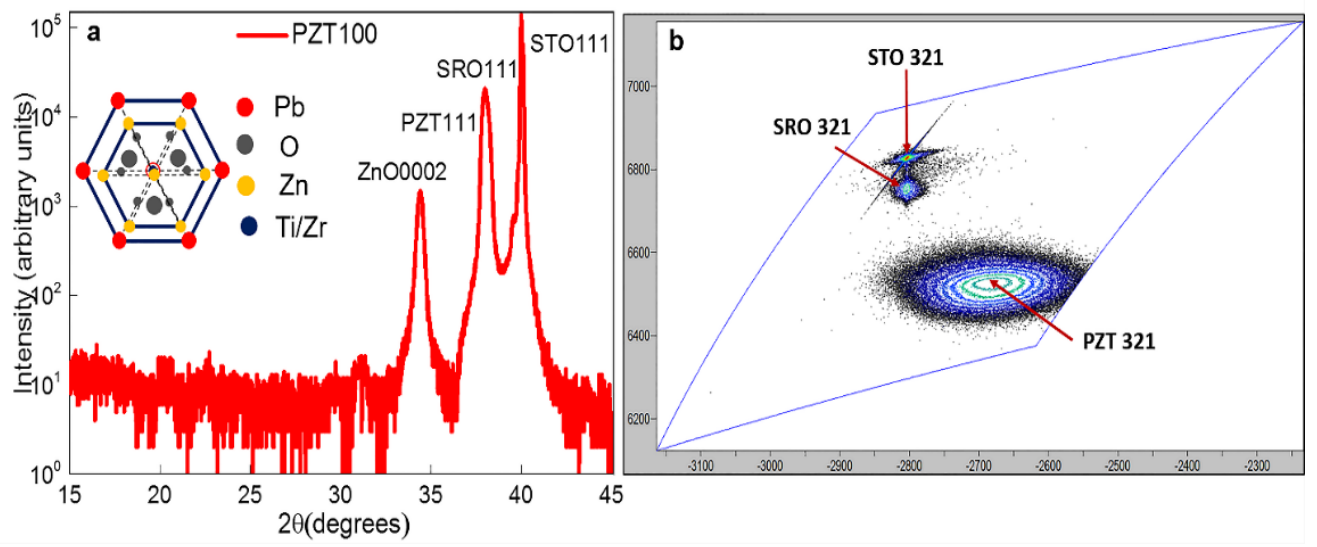

Figure 2.3 (a) The X-ray diffraction spectrum $\theta-2 \theta$ scan of the PZT100 heterostructure around the (111) reflections. (b) The reciprocal space map of the heterostructure around the (321) reflections of the heterostructure. 


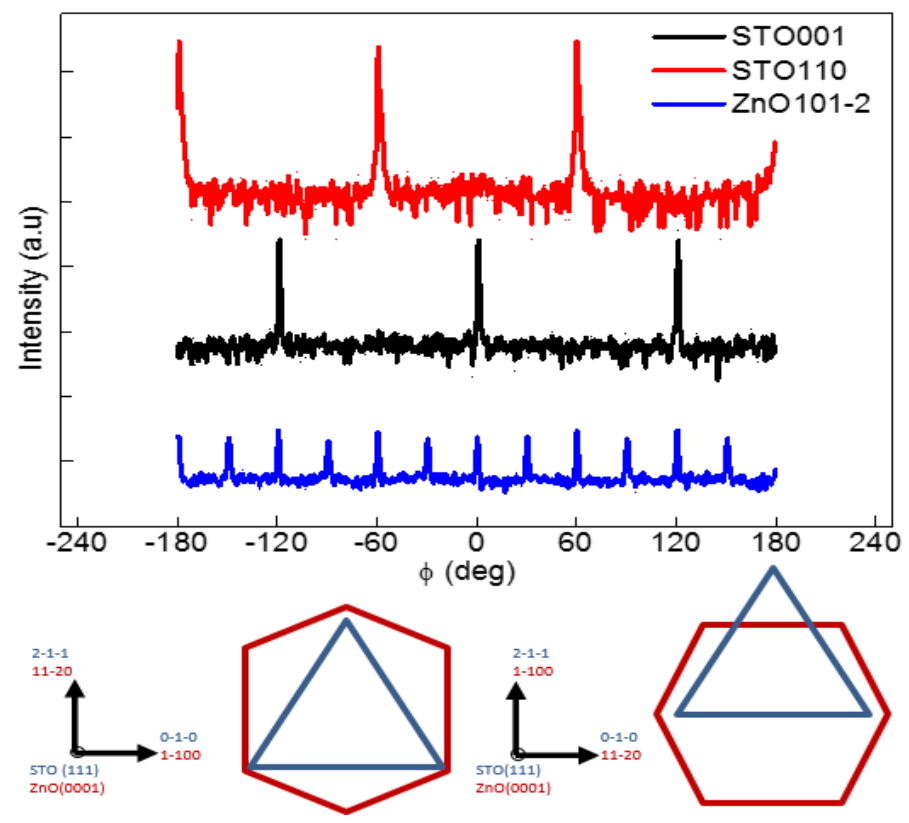

Figure 2.4 In plane $\phi$ scans of $\mathrm{ZnO} 101-2$ plane and STO 110 and 001 planes. The schematic shows the two relative in plane relation between STO 111 and ZnO 0002 planes

\section{Atomic force microscopy}

The surface roughness of the structures were measured using a Bruker Icon AFM. Different thicknesses of ZnO were grown on a thin layer of SRO $(10 \mathrm{~nm}) /$ PZT $(10 \mathrm{~nm})$ to study the roughness of the $\mathrm{ZnO}$ layers. The average rms roughness of the SRO/PZT layer was $2 \mathrm{~nm}$ over an area of $5 \mu \mathrm{m} \times 5 \mu \mathrm{m}$. The rms roughness of the $\mathrm{ZnO}$ layer was $4 \mathrm{~nm}$ and was almost invariant with the thickness of $\mathrm{ZnO}$. Below the AFM micrographs (Figure 2.5) for PZT25, PZT50 and PZT100 are shown. It signifies that the disorder due to the roughness of ZnO remains almost similar for all the $\mathrm{ZnO}$ heterostructure samples. 


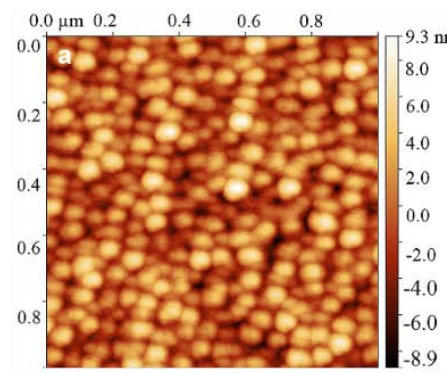

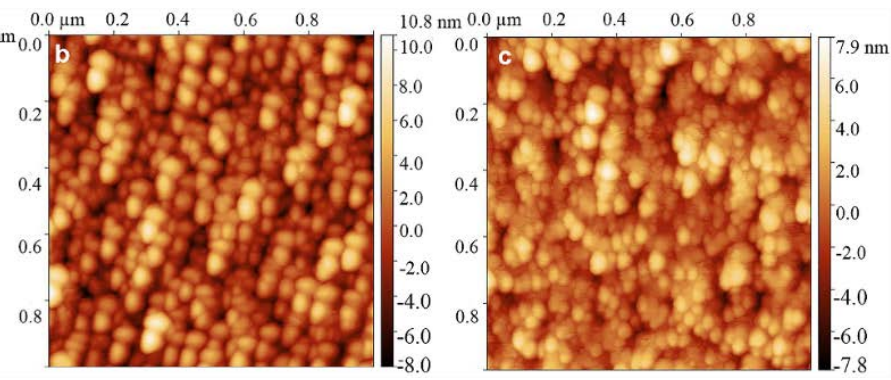

(a)

(b)

(c)

Figure 2.5 AFM topography scans of the surface of (a) $25 \mathrm{~nm} \mathrm{ZnO} \mathrm{(b)} 50 \mathrm{~nm} \mathrm{ZnO}$ and (c) 100
$\mathrm{~nm} \mathrm{ZnO} \mathrm{films} \mathrm{used} \mathrm{in} \mathrm{our} \mathrm{studies,} \mathrm{showing} \mathrm{a} \mathrm{root-mean-square} \mathrm{roughness} \mathrm{of} \sim 4 \mathrm{~nm}$

\section{Transmission electron microscopy}

The local structure and interface layer was probed using a Philips CM300ST FEG Transmission electron microscope (TEM). The cross sectional TEM images showed sharp PZT-ZnO interfaces with no signs of inter-diffusion. Fast Fourier transform (FFT) of the images showed that the PZT was oriented along the 111 direction and $\mathrm{ZnO}$ along the 0001 direction. The FFT pattern also reveals the inherent six fold symmetry present in the $\mathrm{ZnO}(0001)$ and the PZT (111). The absence of any mixed characteristics in the FFT pattern of $\mathrm{ZnO}$ shows that the two in plane domains of $\mathrm{ZnO}$ are not mixed together. 


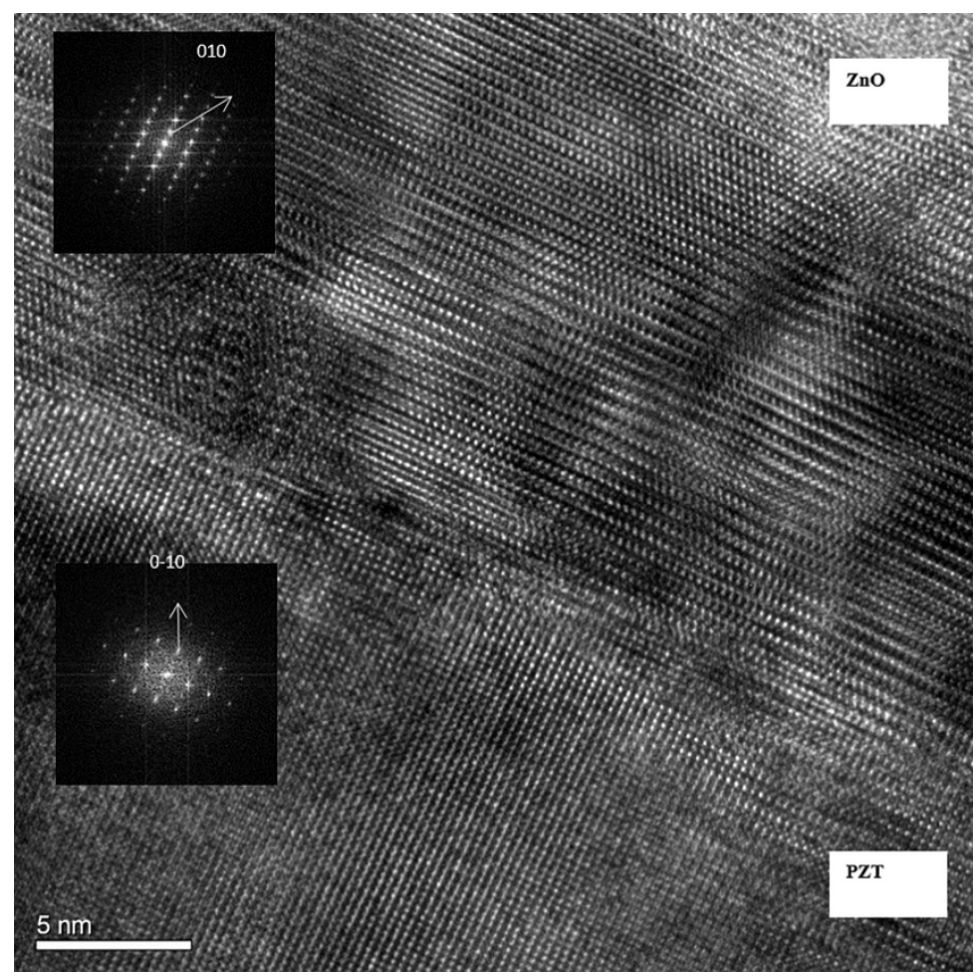

Figure. 2.6 Cross-sectional TEM image of the PZT-ZnO interface PZT100 epitaxial film, the insets show the fast Fourier transform of the image.

\subsection{Conclusions}

In this chapter the pulsed laser deposition process and electrode patterning process has been briefly described. Additionally, the structural characterizations using XRD, AFM, and TEM have been provided. It is shown that epitaxial heterostructures of SRO/PZT/ZnO/SRO has been grown using pulsed laser deposition. Different capacitor structures have been fabricated using photo-lithography and etching. TEM results showed that the interfaces are sharp with no inter-diffusion. These heterostructures will be used in the thesis in succeeding chapters to manipulate the polarization switching dynamics of PZT through polarization coupling. The ability to grow $\mathrm{ZnO}$ of different thicknesses with a similar rms thickness provides us the opportunity to manipulate the disorder (induced by the roughness) to order 
ratio. This relative disorder value will be utilized later in the thesis to manipulate the overall switching statistics. 


\subsection{References}

1. D. B. Chrisey and G. K. Hubler, Pulsed laser deposition of thin films. (1994).

2. R. Eason, Pulsed laser deposition of thin films: applications-led growth of functional materials. (John Wiley \& Sons, 2007).

3. K. Orsel, R. Groenen, B. Bastiaens, G. Koster, G. Rijnders and K.-J. Boller, APL materials 3 (10), 106103 (2015).

4. R. Groenen, J. Smit, K. Orsel, A. Vailionis, B. Bastiaens, M. Huijben, K. Boller, G. Rijnders and G. Koster, APL materials 3 (7), 070701 (2015).

5. H. M. Smith and A. Turner, Applied Optics 4 (1), 147-148 (1965).

6. D. Dijkkamp, T. Venkatesan, X. Wu, S. Shaheen, N. Jisrawi, Y. Min-Lee, W. McLean and M. Croft, Applied Physics Letters 51 (8), 619-621 (1987).

7. G. J. Rijnders, G. Koster, D. H. Blank and H. Rogalla, Applied physics letters 70 (14), 18881890 (1997).

8. R. Ramesh and D. G. Schlom, MRS bulletin 33 (11), 1006-1014 (2008).

9. G. Koster, G. J. Rijnders, D. H. Blank and H. Rogalla, Applied physics letters 74 (24), 37293731 (1999).

10. E. Bousquet, M. Dawber, N. Stucki, C. Lichtensteiger, P. Hermet, S. Gariglio, J.-M. Triscone and P. Ghosez, Nature 452 (7188), 732-736 (2008).

11. H. Y. Hwang, Y. Iwasa, M. Kawasaki, B. Keimer, N. Nagaosa and Y. Tokura, Nature materials 11 (2), 103-113 (2012).

12. D. H. Lowndes, D. Geohegan, A. Puretzky, D. Norton and C. Rouleau, Science 273 (5277), 898 (1996).

13. X. Gao, L. Liu, B. Birajdar, M. Ziese, W. Lee, M. Alexe and D. Hesse, Advanced Functional Materials 19 (21), 3450-3455 (2009).

14. R. K. Singh and J. Narayan, Physical Review B 41 (13), 8843-8859 (1990).

15. M. D. Nguyen, M. Dekkers, E. Houwman, R. Steenwelle, X. Wan, A. Roelofs, T. SchmitzKempen and G. Rijnders, Applied Physics Letters 99 (25), 252904 (2011).

16. M. Haun, E. Furman, S. Jang and L. Cross, Ferroelectrics 99 (1), 13-25 (1989).

17. J. F. Scott, Ferroelectric memories. (Springer Science \& Business Media, 2000). 
18. U. Özgür, Y. I. Alivov, C. Liu, A. Teke, M. A. Reshchikov, S. Doğan, V. Avrutin, S. J. Cho and H. Morkoç, Journal of Applied Physics 98 (4), 041301 (2005).

19. G. Koster, B. L. Kropman, G. J. Rijnders, D. H. Blank and H. Rogalla, Applied Physics Letters 73 (20), 2920-2922 (1998).

20. M. Birkholz, Thin film analysis by X-ray scattering. (John Wiley \& Sons, 2006).

21. X. H. Wei, Y. R. Li, W. J. Jie, J. L. Tang, H. Z. Zeng, W. Huang, Y. Zhang and J. Zhu, Journal of Physics D: Applied Physics 40 (23), $7502-7507$ (2007).

22. C. Jia, Y. Chen, X. Liu, S. Yang, W. Zhang and Z. Wang, Nanoscale research letters 8 (1), 18 (2013). 
CHAPTER 3

\section{TUNABLE AND TEMPORALLYSTABLE FERROELECTRIC IMPRINTTHROUGH POLARIZATION COUPLING ${ }^{1}$}

In this Chapter a method is demonstrated to the tune ferroelectric imprint, which is stable in time. This imprint is based on the coupling between the non-switchable polarization of $\mathrm{ZnO}$ and switchable polarization of $\mathrm{PbZr}_{x} \mathrm{Ti}_{(1-x)} \mathrm{O}_{3}$. $\mathrm{SrRuO}_{3} / \mathrm{PbZr}_{x} \mathrm{Ti}_{(1-}$ ${ }_{x} \mathrm{O}_{3} / \mathrm{ZnO} / \mathrm{SrRuO}_{3}$ heterostructures were grown with different $\mathrm{ZnO}$ thicknesses. It is shown that the coercive voltages and ferroelectric imprint varies linearly with the thickness of $\mathrm{ZnO}$. It is also demonstrated that the ferroelectric imprint remains stable with electric field cycling and electric field stress assisted aging.

1 This chapter has been published in: APL Materials 4, 066103 (2016) 


\subsection{Introduction}

Ferroelectric devices based on metal-ferroelectric-metal (MFM) and metal-ferroelectricsemiconductor (MFS) heterostructures are interesting both from the application point of view e.g. in memories and other digital logic as well as for understanding the fundamental physics ${ }^{1-7}$. Traditionally, the ferroelectric random access memory (FeRAM) and the ferroelectric field effect transistor (FeFET) have always been major fields of application ${ }^{2-7}$. Recently, a lot of focus is also being given to the ferroelectric resistive memory (RRAM) based on the ferroelectric tunnel junction (FTJ) and switchable diode effects because of their nonvolatility, non-destructive and fast switching characteristics ${ }^{8,9}$. In the case of ferroelectric based RRAMs the underlying mechanism is the modification of the barrier heights at the two opposite electrodes by switching of its polarization ${ }^{9}$. In the case of the FeFET, it is the modification of the carrier concentration in the gate channel by using the switchable polarization charge of the ferroelectric ${ }^{6-11}$. In both cases the interaction between the switchable polarization charge at the interface and the semiconductor charge carriers plays a major role. Therefore, it is not only important to study the effect of the ferroelectric on the semiconducting properties of the system, but also how the ferroelectric switching properties are modified by the semiconducting properties, because of the depolarization charges. It will also be of interest to see if the ferroelectric switching properties can be modified to our advantage for specific purposes ${ }^{10}$. Analyzing the dynamics of switching should give insight into the actual operating speed, operating voltage as well as the reliability of the MFS system.

In a FeFET the biggest challenge is having relatively long polarization retention times, which is important for realistic applications ${ }^{11-13}$. In such a device the ferroelectric is in contact with a semiconductor, which has a finite screening length as compared to the Thomas-Fermi screening length in a metal) which gives rise to a finite depolarizing field. Because of the depolarizing effect arising from this incomplete screening, the ferroelectric polarization is not stable for long time periods ${ }^{12,13}$. It would be of great significance if one could make an FeFET in which the polarization is not lost by pinning it in one direction, thus stabilizing the ferroelectric polarization in one direction. This can be manifested by an imprint in the 
polarization-electric field ( $\mathrm{P}-\mathrm{E})$ hysteresis loop. This will lead to a longer retention time of the ferroelectric in the FeFET device, making it suitable for practical applications.

In the case of kinetic memories e.g. phase change memory and RRAM the basic concept is to make two thermodynamically inequivalent stable states with different electrical properties 7,814 . This would enable a stable electrical resistance over a long time. One way to achieve the creation of two thermodynamically inequivalent polarization states is by coupling a nonswitchable polarization vector to a switchable polarization vector. This method is much faster and more reliable compared to the destructive switching method of writing and reading ${ }^{7-9}$.

Another application, in which ferroelectric imprint tuning is important is in the case of Microelectromechanical switch (MEMS) devices. It was recently shown that imprint in P-E hysteresis loop can be used advantageously to achieve a high figure of merit (the figure of merit is inversely proportional to the dielectric permittivity of the ferroelectric at $0 \mathrm{~V}$ ) in MEMS devices ${ }^{15}$. However in this case the imprint was neither temporally stable nor was it tunable.

Ferroelectric imprint has been one of the main features of FeRAM devices ${ }^{2,3}$. The asymmetry of the ferroelectric loop has been explained by several mechanisms, such as the presence of a ferroelectrically dead layer, a ferroelectric-electrode rectifying contact, by defect domain pinning or by interface charge injection. But none of these imprint phenomena have been shown to be temporally stable, nor have been demonstrated for tunability. Furthermore, the observed imprint appears to be very much processing dependent ${ }^{16-20}$. For all the above cases, it would be helpful if one could devise a method to create a ferroelectric imprint which is temporally as well as stable on multiple cycling, is tunable and process independent. Moreover, if we can device a methodology by which we can tune the electric field at the ferroelectric-electrode interface we can also analyze the effect of the gradual increase of the interface electric field on the switching dynamics of a ferroelectric (Discussed in detail in Chapter 4). 


\subsection{Results and Discussion}

\subsubsection{Theory}

In this paper a simple method to tune the imprint of a ferroelectric which is temporally stable and in principle should be process independent is reported. To achieve the goal of stabilizing one state of polarization over another, heterostructures of $\mathrm{ZnO}$ and $\mathrm{PbZ}_{0.58} \mathrm{Ti}_{0.42} \mathrm{O}_{3}(\mathrm{PZT}(58 / 42))$ as a model system were chosen. $\mathrm{PZT}(58 / 42)$ in the rhombohedral phase (space group R3m) has a ferroelectric polarization along the $(111)_{c}$ direction ${ }^{2}$. ZnO in its parent wurtzite structure (space group $\mathrm{P}_{3} \mathrm{~cm}$ ) has a non-switchable polarization along the $(0001)_{h}$ direction ${ }^{21,22}$. Growing the PZT along the (pseudo) cubic (111) direction provides us with the unique opportunity to study the interaction between the switchable and nonswitchable polarization at the interface (Figure 3.1(a)). Because of the non-switchable polarization of $\mathrm{ZnO}$ one polarization state of PZT should be more stable than the other. The electric field due to the dipole is directly proportional to the thickness of the layer $\left(d_{z n o}\right)$ and its in-built polarization $\left(\mathrm{P}_{\mathrm{ZnO}}\right)^{23}$. Hence, it would be very important to see how the ferroelectric imprint varies with the thickness of the $\mathrm{ZnO}$. The thickness of the nonswitchable $\mathrm{ZnO}$ layer is $\mathrm{dzno}$ and the thickness of the switchable PZT is dpzt. The dielectric permittivity and polarization of $\mathrm{ZnO}$ and PZT are $\varepsilon_{\mathrm{ZnO}}, \varepsilon_{\mathrm{PZT}}$ and $\mathrm{P}_{\mathrm{ZnO}}$, $\mathrm{P}_{\mathrm{PZT}}$ respectively. Applying the condition of the continuity of the electric displacement field across the PZT/ZnO interface and calculate the applied voltage $V_{a p p}$ across the layer stack we can write

$$
\begin{gathered}
P_{P Z T}+\varepsilon_{0} \varepsilon_{P Z T} E_{P Z T}=P_{Z n O}+\varepsilon_{0} \varepsilon_{Z n O} E_{Z n O} \\
V_{a p p}=E_{P Z T} d_{P Z T}+E_{Z n O} d_{Z n O}
\end{gathered}
$$

$E_{Z n O}, E_{p Z T}$ are the electric fields across the piezoelectric and the ferroelectric respectively and $V_{\text {app }}$ is the applied voltage. In order to find the offset voltage $V_{\text {off, }}$ i.e. the difference in voltage that can produce the same amount of switching for both the biases we need to balance the $V_{P Z T}$ for both the biases. The intrinsic coercive field of the PZT will remain the same only the applied voltage across the PZT will get changed for opposite biases due to the built in field. Following this we arrive at 


$$
\begin{gathered}
V_{P Z T}=V_{a p p}-V_{o f f}+d_{Z n O}\left(P_{P Z T}+\varepsilon_{0 \varepsilon P Z T} E_{P Z T}\right) / \varepsilon_{0} \varepsilon_{Z n O} \\
V_{o f f}=P_{Z n o d} d_{Z n O} / \varepsilon_{0} \varepsilon_{Z n O}
\end{gathered}
$$

It can be seen from the Equation 4 that the imprint $\left(V_{\text {off }}\right)$ is linearly dependent on the thickness of the non-switchable $\mathrm{ZnO}$. In order to obtain a high imprint the non-switchable material should have a high polarization and a low dielectric constant. On the other hand a low dielectric constant material will also increase the operating voltage, as is evident from Equation 3. We can also see from Equation 3. $\left(d_{Z n O}\left(P_{P Z T}+\varepsilon_{0} \varepsilon_{P Z T} E_{P Z T}\right) / \varepsilon_{0} \varepsilon_{Z n O}\right)$ the presence of $\mathrm{ZnO}$ also leads to a symmetric increase (with respect to voltage) of coercive voltage with $\mathrm{ZnO}$ thickness. In our case the dpzt is fixed at $1 \mu \mathrm{m}$, whereas $d z n o$ is varied from $25 \mathrm{~nm}$ to $150 \mathrm{~nm}$. However, it must be mentioned here that the presence of bound charges would make the up-state polarization unstable if not compensated by free charge carriers. The presence of free charge carriers will however modify the effective field at the interface between the switchable ferroelectric PZT and nonswitchable ZnO layer. The presence of free charge carriers can in effect lead to the accumulation of free charge carriers at the PZT-ZnO interface. If, $\sigma$ represents the trapped free charge carriers then

$$
V_{o f f}=\left(P_{Z n O}+\sigma\right) d_{Z n O} / \varepsilon_{0} \varepsilon_{Z n O}
$$

It will in effect lead to a decrease of $\mathrm{V}_{\text {off }} 18,24,25$.

\subsubsection{Experiment}

In this chapter a simple concept is reported to tune the imprint of a ferroelectric which is temporally stable and in principle should be process independent. Capacitor structures of $\mathrm{SrRuO}_{3}(80 \mathrm{~nm}) / \mathrm{PZT}(58 / 42)(1000 \mathrm{~nm}) / \mathrm{ZnO}(25-150 \mathrm{~nm}) / \mathrm{SrRuO}_{3}(80 \mathrm{~nm})$ were fabricated on $\mathrm{STiO}_{3}(111)$ substrates using pulsed laser deposition followed by photolithography and etching ${ }^{26}$. For the purpose of tuning the ferroelectric imprint devices with four different thicknesses of $\mathrm{ZnO}, 25 \mathrm{~nm}, 50 \mathrm{~nm}, 100 \mathrm{~nm}$ and $150 \mathrm{~nm}$ as well as a device without $\mathrm{ZnO}$, were fabricated. Hereon, these samples are named PZT25, PZT50, PZT100, PZT150 and PZT0, respectively. The $\mathrm{SrRuO}_{3}$ and $\mathrm{PZT}$ grow epitaxially on $\mathrm{SrTiO}_{3}$ in the $(111)_{c}$ direction whereas ZnO grows preferentially in the $(0001)_{h}$ direction. The ferroelectric characterization was 
carried out at room temperature using the aixACCT 3000 TF Analyzer set up. As mentioned in the previous chapter from the reciprocal space map it was seen that the PZT films are fully strain relaxed.

In order to measure the tuning of the ferroelectric hysteresis with the $\mathrm{ZnO}$ thickness, the PE hysteresis loop for all samples are analyzed. In Figure 3.1 (b) the P-E hysteresis loops of the PZTO and PZT100 devices measured at $1000 \mathrm{~Hz}$ is shown. It is observed that for the device without ZnO layer (PZTO) the saturation polarization $\left(P_{s}\right)$ is around $34 \mu \mathrm{C} / \mathrm{cm}^{2}$ and the coercive fields are approximately $\pm 3 \mathrm{MV} / \mathrm{m}$. In the case of the PZT100 sample the saturation polarization was $34 \mathrm{\mu C} / \mathrm{cm}^{2}$ and the coercive fields were $-9.33 \mathrm{MV} / \mathrm{m}$ and $21.55 \mathrm{MV} / \mathrm{m}$ respectively for the negative and positive bias.
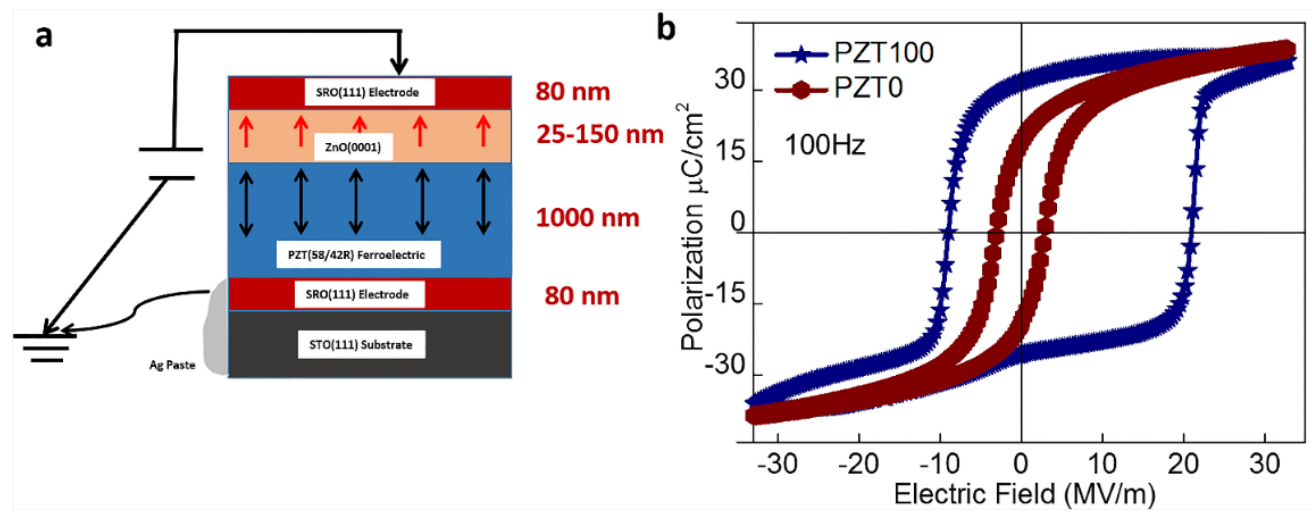

Figure 3.1 Schematic of the devices and the ferroelectric P-E hysteresis loop measured at $100 \mathrm{~Hz}$ of the PZT and PZT100. a) Heterostructure device used for imprint control. The thickness of the PZT was kept fixed at $1 \mu \mathrm{m}$ and the $\mathrm{ZnO}$ thickness was varied between $25 \mathrm{~nm}$ and $150 \mathrm{~nm}$. b) The figure shows significant imprint for the PZT100 sample as well as opening up of the loop due to increase in the coercive fields.

In Figure 3.2. the coercive fields for the opposite biases for all samples the hysteresis loop opens up with increasing thickness of $\mathrm{ZnO}$, and the coercive voltages also increase linearly with $\mathrm{ZnO}$ thickness according to Equation 3. 


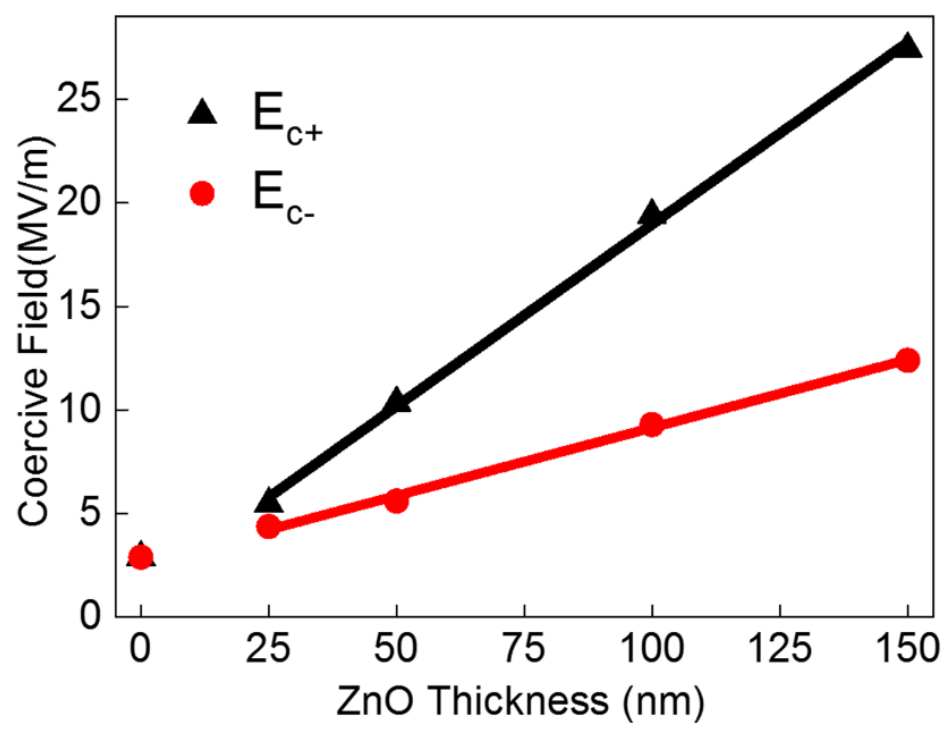

Figure 3.2 Coercive field as function of $\mathrm{ZnO}$ thickness. This figure shows the linear variance of the coercive fields with the thickness of the $\mathrm{ZnO}$ layer measured at $100 \mathrm{~Hz}$.

To see the actual tunability of the imprint due to the fixed polarization of $\mathrm{ZnO}$ in Figure 3.3 the offset voltage $\left(V_{\text {off }}\right)$ (imprint $\left.\left(V_{c+}+V_{c-}\right) / 2\right)$ (Equation 3$)$ is plotted. We can see that the $V_{\text {off }}$ is linearly proportional to the thickness of $\mathrm{ZnO}$ as in Equation 3. The coercive voltage was nearly independent of the measurement frequency $(10 \mathrm{~Hz}-10 \mathrm{kHz}){ }^{3,27}$. Defect dynamics involving charging/discharging of the defect states result in large frequency dispersion of the hysteresis loops in ferroelectrics ${ }^{3}, 27,28$. This indicates that our electrical measurements are not dominated by defects and other relaxation mechanisms. This frequency dispersion study of our ferroelectric hysteresis loop shows our measurements are not dominated by artefacts resulting from leakage, and other space charge and other relaxation mechanisms and we are measuring the intrinsic switching characteristic of the system ${ }^{3,27-29}$. The voltage offset per unit $\mathrm{nm}$ of $\mathrm{ZnO}$ is $0.05 \mathrm{~V}$ as determined from the slope of the imprint versus $\mathrm{ZnO}$ thickness plot. . In our case the PZT is in contact with SRO on one side and ZnO on another side which gives rise to different built-in voltages ${ }^{30}$. The offset voltage due to the different built-in voltages can be written as $V_{d e p}{ }^{\text {off }} \propto V_{d 1}-V_{d 2}$, where $V_{d 1}, V_{d 2}$ are the two built-in voltages across the PZT. The value $\left(V_{d 1}-V_{d 2}\right) / 2$ was found to be (using temperature dependent currentvoltage measurements shown in Chapter 5) -0.4.V, which is in the order of what is normally 
observed in ferroelectric thin films. However the value of this built-in voltage is much less than imparted by the $\mathrm{ZnO}$ layer and is constant for all the samples and doesn't scale with the $\mathrm{ZnO}$ thickness. To ensure that the imprint effect is not dominated by the depletion effect because of the presence of two different materials across the PZT we measured the imprint for a $500 \mathrm{~nm}$ thick PZT with a $100 \mathrm{~nm}$ ZnO on top of it. In case the imprint is dominated by a depletion effect it should scale inversely with the thickness of PZT which was not observed (Figure 3.3 inset). Furthermore, symmetrical capacitance voltage measurements also showed there was no significant depletion layer formation inside the ZnO layer. To understand the effect of the free charge carriers in $\mathrm{ZnO}$ as described by Equation. 5, all the samples were annealed at $600^{\circ} \mathrm{C}$ for 15 mins in $\mathrm{O}_{2}$ atmosphere to decrease the free charge carriers in $\mathrm{ZnO}^{31,32}$. We can see that upon annealing (Figure 3.3) the slope of the imprint vs $\mathrm{ZnO}$ thickness increases according to Equation. 5. This shows that a more insulating ZnO layer would be more efficient for inducing imprint.

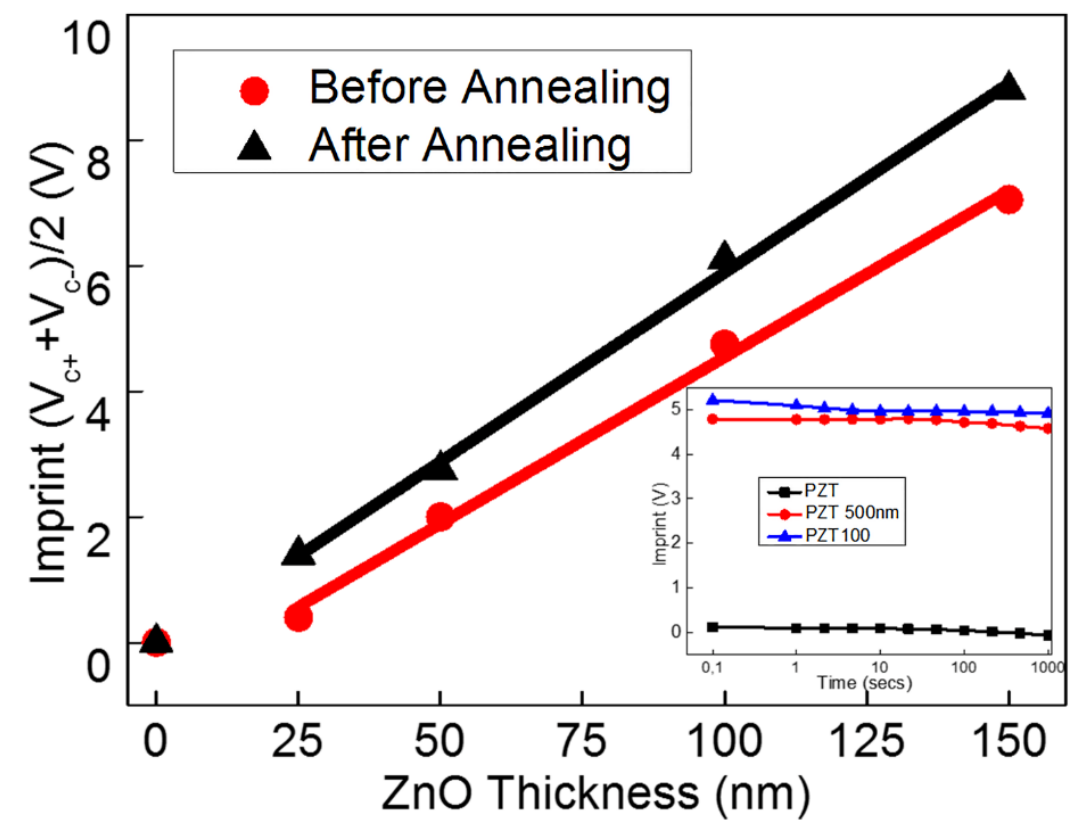

Figure 3.3 Tuning of the imprint and with the $\mathrm{ZnO}$ thickness and the effect of annealing. Imprint (Offset voltage) of PZT (thickness fixed at $1 \mu \mathrm{m}$ ) samples measured as a function of $\mathrm{ZnO}$ thickness $(25 \mathrm{~nm}, 50 \mathrm{~nm}, 100 \mathrm{~nm}, 150 \mathrm{~nm})$. The figure shows that the imprint varies linearly with the thickness of $\mathrm{ZnO}$. It can be seen upon annealing the imprint increases but still varies linearly. The inset shows the time dependent imprint behaviour of the PZT, PZT100 and PZT 500nm sample with $100 \mathrm{~nm} \mathrm{ZnO}$ on top. 
To make sure that the imprint is stable upon aging a cyclical voltage of $\pm 35 \mathrm{~V}$ was applied at $1 \mathrm{~Hz}$ frequency for up to 1000 secs. It was seen that even after 1000 secs of cycling the imprint didn't change by more than $4 \%$ for all the samples.

Waser et al 16,17 showed that the imprint in ferroelectrics is caused by an interface field at the ferroelectric-electrode interface arising due to the finite separation between the polarization and screening charges. This interface field leads to charge injection from the electrode into the film which gets trapped at the interface and since the ferroelectric switching time is much faster than the charge detrapping time these trapped charges at the interface give rise to an imprint increasing with time. In case when the imprint arises due to the interface field which is along the direction of polarization, upon poling the sample in one direction, the samples prefer that state due to electrical stress because of more charge injection and trapping ${ }^{17,20}$. On application of a constant electric field along (or opposite to) the direction of this built-in field would lead to an increase (decrease) of the imprint.

To test the role of the above mechanism role how the imprint evolves with time and also the effect of a constant voltage along to the direction of the imprint on the voltage shift was investigated. In Figure 3.4 we can see that for all the samples the imprint remains almost constant with time with which shows that the imprint is very stable to the effect of interface field. In Figure 3.4 inset shows that for PZT100 the application of a constant voltage leads to a very small change in the imprint as compared to the $0 \mathrm{~V}$ as a function of aging time. As a matter of fact, it's seen that the imprint actually decreases with time, indicating that the direction of the induced imprint is opposite to the direction of the interface field. Similar trends were also observed for all the PZT25 and PZT50 but for PZTO the imprint increased with time as was also observed by Waser et $a^{16}$. These observations show that the role of interface screening in inducing imprint is very small compared to that induced by the $\mathrm{ZnO}$ layer. Also, the imprint induced by interface field increase with the temperature which was not observed in our case, on the contrary the imprint increased with for lower temperatures. 


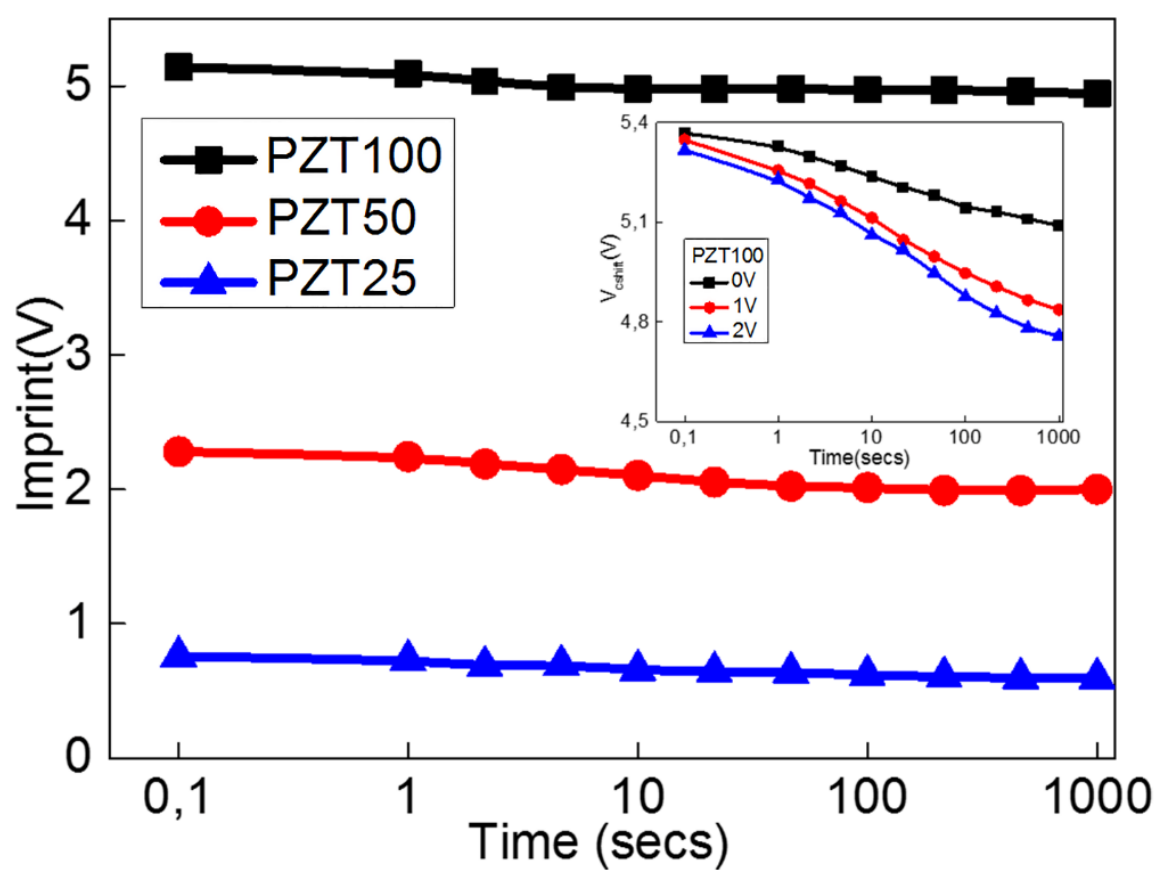

Figure 3.4 Stability of Imprint with aging. Stability of the imprint for all the samples with aging. Inset shows the evolution of the imprint for PZT100 upon application of a constant field along the direction of the imprint, the decrease in imprint with the voltage shows that the direction of the imprint induced by the $\mathrm{ZnO}$ layer is opposite to that of the interface field.

\subsection{Conclusions}

In conclusion, it is shown that the imprint or the offset voltage in a ferroelectric can be controlled and analyzed the underlying mechanism behind this phenomenon. Control of ferroelectric imprint is necessary for the FeFET which has very short retention time as well as for RRAM based kinetic memories where it is necessary to make one state more stable than the other. Moreover, tunable imprint in a ferroelectric, which is also stable over time can lead to higher figures of merit and stable performance in energy harvesters. Our results have shown that the imprint is directly proportional to the thickness of the $\mathrm{ZnO}$ layer thickness. This simple and robust method of gradually tuning the electric field at the ferroelectric-electrode interface is used in the succeeding chapters to modify the switching dynamics of the ferroelectric PZT film. 


\subsection{References}

1. S. Mathews, R. Ramesh, T. Venkatesan, and J. Benedetto, Science 276(5310), 238-240 (1997).

2. C. A. Paz De Araujo, L. D. McMillan, B. M. Melnick, J. D. Cuchiaro and J. F. Scott, Ferroelectrics 104 (1), 241-256 (1990).

3. J. F. Scott, Ferroelectric memories. (Springer Science \& Business Media, 2000).

4. W. Shockley, Proceedings of the IRE 40 (11), 1365-1376 (1952).

5. Y. Watanabe, Applied Physics Letters 66 (14), 1770 (1995).

6. S. Y. Wu, Ferroelectrics 11 (1), 379-383 (1976).

7. S. Mathews, R. Ramesh, T. Venkatesan and J. Benedetto, Science 276 (5310), 238-240 (1997).

8. T. Choi, S. Lee, Y. Choi, V. Kiryukhin and S.-W. Cheong, Science 324 (5923), 63-66 (2009).

9. D. S. Jeong, R. Thomas, R. Katiyar, J. Scott, H. Kohlstedt, A. Petraru and C. S. Hwang, Reports on Progress in Physics 75 (7), 076502 (2012).

10. A. Ghosh, G. Koster and G. Rijnders, Advanced Functional Materials (2016).

11. J. Hoffman, X. Pan, J. W. Reiner, F. J. Walker, J. P. Han, C. H. Ahn and T. P. Ma, Advanced materials 22 (26-27), 2957-2961 (2010).

12. T. Ma and J.-P. Han, Electron Device Letters, IEEE 23 (7), 386-388 (2002).

13. P. Wurfel and I. P. Batra, Physical Review B 8 (11), 5126-5133 (1973).

14. A. Chanthbouala, V. Garcia, R. O. Cherifi, K. Bouzehouane, S. Fusil, X. Moya, S. Xavier, H. Yamada, C. Deranlot, N. D. Mathur, M. Bibes, A. Barthelemy and J. Grollier, Nature materials 11 (10), 860-864 (2012).

15. S. Baek, J. Park, D. Kim, V. Aksyuk, R. Das, S. Bu, D. Felker, J. Lettieri, V. Vaithyanathan and S. Bharadwaja, Science 334 (6058), 958-961 (2011).

16. M. Grossmann, O. Lohse, D. Bolten, U. Boettger, T. Schneller and R. Waser, Journal of Applied Physics 92 (5), 2680 (2002).

17. M. Grossmann, O. Lohse, D. Bolten, U. Boettger and R. Waser, Journal of Applied Physics 92 (5), 2688 (2002).

18. A. K. Tagantsev and G. Gerra, Journal of Applied Physics 100 (5), 051607 (2006). 
19. A. K. Tagantsev, I. Stolichnov, N. Setter and J. S. Cross, Journal of Applied Physics 96 (11), 6616 (2004).

20. W. L. Warren, B. A. Tuttle, D. Dimos, G. E. Pike, H. N. Al-Shareef, R. Ramesh and J. T. Evans, Japanese journal of applied physics 35 (2S), 1521 (1996).

21. V. M. Voora, T. Hofmann, M. Brandt, M. Lorenz, M. Grundmann, N. Ashkenov, H. Schmidt, N. lanno and M. Schubert, Physical Review B 81 (19) (2010).

22. A. Janotti and C. G. Van de Walle, Reports on Progress in Physics 72 (12), 126501 (2009). 23. D. J. Griffiths and R. College, Introduction to electrodynamics. (prentice Hall Upper Saddle River, NJ, 1999).

24. K. Abe, N. Yanase, T. Yasumoto and T. Kawakubo, Japanese Journal of Applied Physics41 (Part 1, No. 10), 6065-6071 (2002).

25. K. Abe, N. Yanase, T. Yasumoto and T. Kawakubo, Journal of Applied Physics 91 (1), 323 (2002).

26. M. D. Nguyen, M. Dekkers, E. Houwman, R. Steenwelle, X. Wan, A. Roelofs, T. SchmitzKempen and G. Rijnders, Applied Physics Letters 99 (25), 252904 (2011).

27. L. Pintilie and M. Alexe, Applied Physics Letters 87 (11), 112903 (2005).

28. L. Pintilie, Charge transport in ferroelectric thin films. (INTECH Open Access Publisher, 2011).

29. J. F. Scott, Journal of Physics: Condensed Matter 20 (2), 021001 (2008).

30. S. M. Sze and K. K. Ng, Physics of semiconductor devices. (John wiley \& sons, 2006).

31. H. S. Kang, Journal of Applied Physics 95 (3), 1246 (2004).

32.F. K. Shan, G. X. Liu, W. J. Lee and B. C. Shin, Journal of Applied Physics 101 (5), 053106 (2007). 


\section{CHAPTER 4}

\section{MULTI-STABILITYIN BI-STABLE FERROELECTRIC MATERIALSTOWARDS ADAPTIVE APPLICATIONS ${ }^{1}$}

Traditionally thermodynamically bistable ferroic materials are used for non-volatile operations based on logic gates (e.g. in the form of field effect transistors). But, this inherent bistability in these class of materials limit their applicability for adaptive operations. Emulating biological synapses in real materials necessitates gradual tuning of resistance in a non-volatile manner. Even though in recent years few observations have been made of adaptive devices using a ferroelectric the principal question as to how to make a ferroelectric adaptive has remained elusive in the literature. Here it is shown that, by controlling locally the nucleation energy distribution at the ferroelectric-electrode interface we can make a ferroelectric with addressable multiple states behave as necessary for adaptive applications. This is realized by depositing a layer of non-switchable $\mathrm{ZnO}$ on thin film ferroelectric $\mathrm{PbZr}_{x} \mathrm{Ti}_{(1-x)} \mathrm{O}_{3}$. This methodology of interface engineered ferroelectric should enable us realise brain-like adaptive memory in CMOS devices. Furthermore the temporally stable multistability in ferroelectrics should enable us to design multistate memory and logic devices.

1 This chapter has been published in: Advanced Functional Materials Volume 26, Issue 31 August 16, 2016, Pages 5748-5756 


\subsection{Introduction}

Lately research has focussed on the realization of artificial brain like computation motivated by their ability to learn and perform very complex computations e.g. pattern recognition, which are not viable using present day computers based on the Von Neumann architecture ${ }^{1-10}$. Analog computers could in principle overcome the limitations of digital computers by adaptive processing of information 4 , 10-14. An adaptive system can be defined as one that can dynamically adjust its system parameters rather than having a fixed input to output relation. In the human brain adaptive computation is carried out by the neurons; a neuron communicates with other neurons through synapses and the learning function is derived from the plasticity of the synapse 7,10,13-16. The strength of a neuron connection is determined by the weight of the connection between two neurons which is remembered by the synapse and the ability of the synapse to update itself gives rise to the adaptability (learning ability) $4,6-8,10,14,17$. Recently, a lot of work has been reported on memristor like devices where the internal resistance of the system was modified using an external stimuli ${ }^{18,19}$. In particular switching speed and CMOS compatibility are major issues with the materials used, while the processing of these materials are still under development ${ }^{4,18}$. To summarize, a non-volatile system with multiple valued and tunable internal states (e.g. resistance, polarization, magnetization etc.) but also exhibits stability and endurance of the intermediate states, has a suitable operating temperature, CMOS processing compatibility 4, 20 and sufficient switching speeds needs to be developed.

Ferroelectrics because of their intrinsic non-volatility, fast switching and CMOS compatibility naturally become are a promising candidate for adaptive switching, the bottleneck being its bistability $2,4,21$. The identification of ferroelectrics for adaptive computation in the form of field effect transistor (FeFET) was already proposed by Ishiwara in 1993 22,23 and more recently observed in the form of a tunnel junctions (FTJ) by Barthelemy et $a^{2}{ }^{2}$. In order to quantify the observed adaptive nature of the device the authors approximated the fraction of the switched domains as a summation of five Heaviside step functions without any explicit physical reasoning. What still needs to be answered is the question of how to achieve multi- 
level states and how to tune the number of internal states in an otherwise bi-stable system needed for adaptive computation which has, to our knowledge, never been addressed in literature.

Additionally, it is not only important to be able to adjust the weights but also the rate at which the weights are updated upon each iteration. The neuron learning and weight adjustments are made using a back propagation algorithm and the rate of change of weights (neuron activation function) based on the delta rule determines the learning rate and error 13-15. In an artificial neuron network the neuron activation functions used are usually sigmoidal in nature, the main reason for sigmoidal functions being used is that the derivative of a sigmoidal function being a double sigmoidal function, it makes it suitable for the back propagation method. Activation functions can be broadly classified as hard and soft activation functions. For realizing a discreet neuron (perceptron) a hard activation function is used based on ON and OFF switch, this can be used to solve simple problems very fast however the convergence might not be accurate and are only be used as binary classifiers. For more complex, continuous neurons (multi-layer perceptrons) where learning and accuracy are most important one needs soft activation functions which are generally sigmoidal. The ability of a neural network to learn depends on the number of degrees of freedom available to the network (for an electronic device it means the number of switchable states). The number of degrees of freedom determines the plasticity of the system, i.e., its capability of approximating the training set (plasticity scales with the number of degrees of freedom) 1-9. For a rapid convergence and avoiding over shooting the weights need to be adjusted gradually in small steps and which requires a moderate slope of the activation function. In general the lesser the slope of the polarization switching curve higher will be the plasticity which in turn enhances the learning ability and helps to reduce the training error. The learning ability of the neuron scales inversely with the steepness of the activation functions which determines the plasticity of the neuron. For a optimal performance of a neural network one needs activation functions with different steepness 4, 8-15, 24. So, it is also necessary to reproduce different neuron activation functions in the switching characteristics of the ferroelectric. 
In this work it is shown how we can create multi-level states and also tune the number of states in a ferroelectric thereby tuning the plasticity of the ferroelectric. This can pave the way for ferroelectrics to be utilized for adaptive computation based on different measurable quantities as a function of the switched polarization e.g. in an FeFET or an FTJ 2, 25, 26.

\subsection{Experimental Section}

The ferroelectric characterizations were carried out at room temperature using an aixACCT 3000 TF Analyzer set up. The pulse switching method was employed to measure the switching polarization as function of the voltage and time (Scheme 1). Measurements were carried out on $200 \mu \mathrm{m}$ by $200 \mu \mathrm{m}$ capacitor structures. The write pulse width ( $\mathrm{t}_{3}$ ) ranging from $2.5 \mu \mathrm{s}$ to $1 \mathrm{~s}$, was applied with a $0 \mathrm{~s}$ rise time. In order to make sure that RC time constants did not affect the measurement $50 \times 50 \mu \mathrm{m}^{2}$ and $100 \times 100 \mu \mathrm{m}^{2}$ capacitors was tested, which did not show any difference in switching characteristics. Additionally, a $100 \Omega$ resistor in series was also put to ensure that the switching characteristics is not affected by the RC time constant. The measurements were carried out using a sequence of four pulses. Pulses 1, 3, 4 had the same amplitude of $35 \mathrm{~V}$ and a duration of $25 \mu$ s (See Scheme 1). The amplitude $\mathrm{V}_{2}$ of pulse 2 was varied between $1 \mathrm{~V}$ to $23 \mathrm{~V}$ depending on the coercive voltage, and was varied in the range $2.5 \mu$ s to $1 \mathrm{~s}$. Pulse 1 defines the state of polarization of the ferroelectric, pulse 2 (write pulse) switches the polarization, pulse 3 measures the switched part of the polarization defined by pulse 2 and from pulse 4 the non-switching polarization is obtained. As have been mentioned in the manuscript in case of WURD (write up read down), $V_{1}$ is negative, $V_{2}$ is positive, $V_{3}$ and $V_{4}$ are negative, and vice versa for WDRU (write down read up). The measurements pulses were separated by a delay time $t_{1}=1 \mathrm{sec} 29$. A cyclical voltage of $\pm 35 \mathrm{~V}$ was applied at $1 \mathrm{~Hz}$ frequency for up to 1000 secs was applied to all the samples before performing any measurements 49. The switching polarizations were measured for the opposite biases on four different samples PZT, PZT25, PZT50 and PZT100, as described above. 


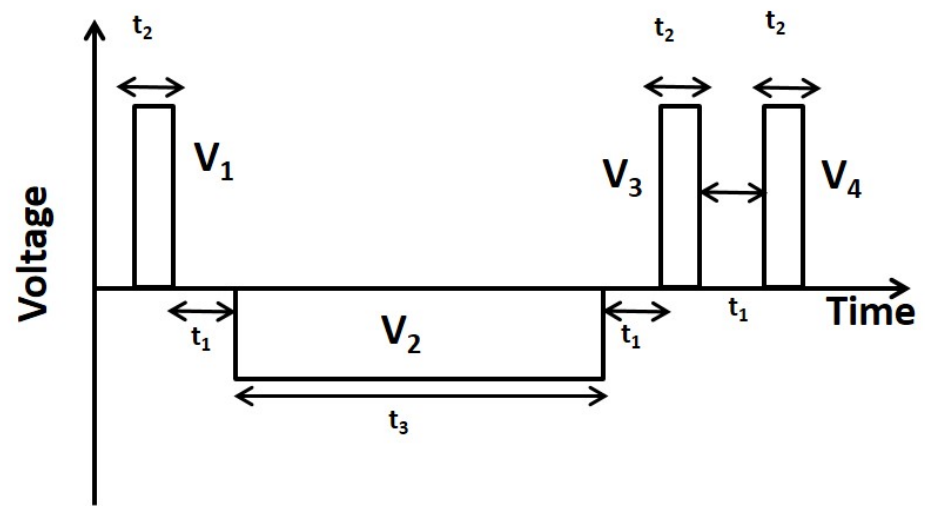

Scheme 1. Sequence of voltage pulses used for measurements of the switching polarization

\subsection{Results and Discussion}

To elaborate on this we first turn to the switching in a uniformly polarized ferroelectric which is explained using Kolmogorov-Avrami-Ishibashi (KAI) model 21,27,28

$$
\begin{aligned}
& \Delta P_{t} / 2 P_{s}=1-\exp \left[-\left(t / t_{0}\right)^{n}\right] \\
& t_{0} \sim \exp \left(E_{a} / E_{\text {ext }}\right)
\end{aligned}
$$

here, $P_{s}$ is the saturated polarization, $\Delta P_{t}$ time dependent change in polarization, $t_{0}$ is the characteristic switching time, $E_{\text {ext }}$ is the electric field, $\mathrm{n}$ the effective domain growth dimension and $E_{a}$ is the effective activation field. As can be seen from the above two equations there is just one unique switching time for a given $E_{\text {ext }}$ and the slope of $\Delta \mathrm{P}_{t} v s$ write time curve is independent of the applied electric field. To explain deviations from the ideal KAl model two different concepts were proposed. Tagantsev et a ${ }^{29}$ proposed that switching kinetics is governed by the statistics of nucleation, whereas Noh et al ${ }^{30}$ and Zhukov et al ${ }^{31}$ took into consideration the statistics of domain growth (dependent on random field variations and pinning centres inside the film). Rappe et al's 32 first principles calculations and subsequent experiments 33 showed that the nucleation and growth activation fields are comparable in magnitude. It can be contended here because of the comparable magnitudes 
of the nucleation and growth activation fields it is very difficult to control the switching by either nucleation or growth alone. Since most of the film actually switches via sideways motion of the domain wall the statistics of growth would always dominate over the nucleation. Tagantsev et al ${ }^{34}$ proposed that the nucleation of opposite domains are stimulated at the ferroelectric-electrode surface. Furthermore Kalinin et al ${ }^{35}$ observed that the nucleation activation energy is extremely sensitive to the built-in electric field near the surface. Hence, it would be more advantageous if we can control the switching by controlling the statistics of nucleation alone rather than the statistics of growth. In epitaxial thin films we can have a substantial control over the interface, in contrast to random field variations inside the film which neither can be controlled nor are dynamically stable.

Taking a cue from the above observations the local electric field at the ferroelectric-electrode surface is controlled and increase the nucleation activation field to a much higher value than the growth activation field and as a result control the switching through nucleation alone. Moreover, we manipulate the distribution of the local electric field by manipulating the normalized roughness (to the thickness) of an interface layer thereby controlling the statistics of the nucleation controlled switching. Controlling the normalized roughness allows one to manoeuvre the fractional variation in the local electric field Figure 4.1 (a). This resulting control over the local distribution of electric fields enables us to create independent regions in the film with individual switching times which in turn enables us to achieve adaptability of the ferroelectric switching by varying the write time for a given electric field Figure 4. 1 (a). 
a

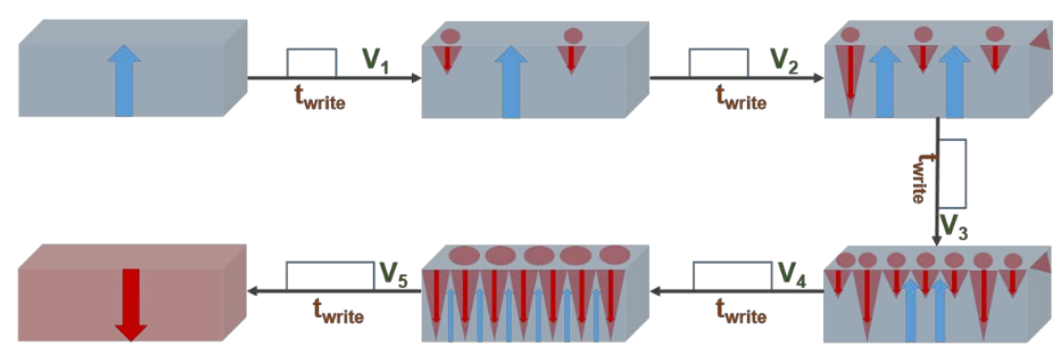

b

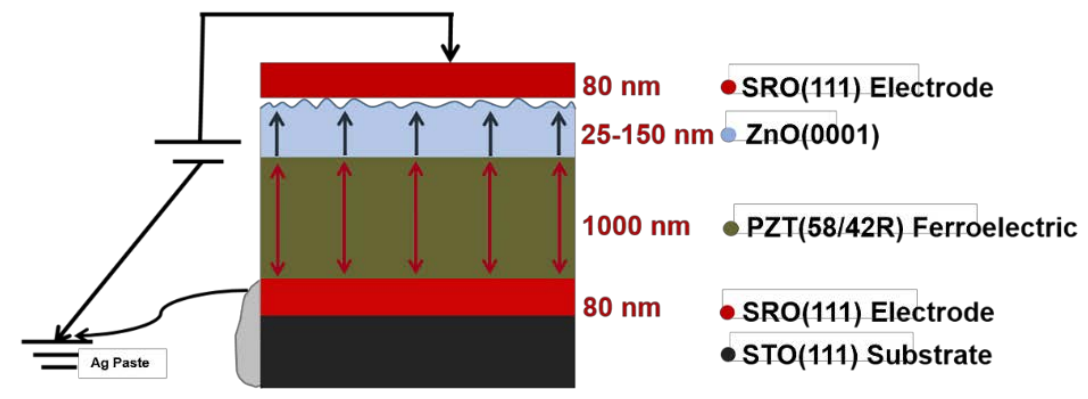

Figure 4.1 Ferroelectric switching for a wide distribution of nucleation switching times a) The schematic shows how a ferroelectric can be switched progressively by applying different electric fields. If the nucleation activation energy at the interface is non- uniformly distributed different regions of the film switches under different applied fields for a given write time or vice versa. b) Heterostructure device used for switching kinetics manipulation. The thickness of the PZT was kept fixed at $1 \mu \mathrm{m}$ and the ZnO thickness was varied from $25 \mathrm{~nm}$ to $150 \mathrm{~nm}$. The non-switchable polarization of $\mathrm{ZnO}$ along $(0001)_{\mathrm{h}}$ couples with the switchable polarization of PZT along $(111)_{c}$. In the schematic shows the average thickness of the $\mathrm{ZnO}$ layer with a finite roughness.

As mentioned in the previous chapter, in order to achieve the goal of increasing the local activation field for switching in a controlled manner heterostructures of $\mathrm{ZnO}$ and $\operatorname{PbZr}(0.58) \mathrm{Ti}_{(0.42)} \mathrm{O}_{3}(\mathrm{PZT})$ as a model system, see Figure 4.1 (b) were fabricated. Lead zirconate titanate (PZT) in rhombohedral phase (space group R3m) has a polarization along the (111) direction ${ }^{21,36}$ and $\mathrm{ZnO}$ (space group $\mathrm{P}_{3} \mathrm{~cm}$ ) in its parent wurtzite structure has a nonswitchable polarization along the (0001) direction ${ }^{37}$. Using Equation 4 Chapter 3 the electric field exerted at the PZT-ZnO interface was tuned by changing the thickness of the nonswitchable $\mathrm{ZnO}$. As shown in Figure 3.2 we can see the $\mathrm{V}_{\text {off }}$ changes linearly with the thickness of $\mathrm{ZnO}$. 
To understand how the non-switchable polarization of $\mathrm{ZnO}$ affect switching kinetics of the PZT it was analysed how the domain nucleation and growth was modified with ZnO thickness. The switching polarization kinetics was analysed using the KAl ${ }^{27,41}$ and NLS ${ }^{29}$ models. The pulse switching method was employed to measure the switching polarization as function of applied field and time 29 (For details of the measurements see Experimental Section). The switching polarizations were measured for the opposite biases on four different samples PZT, PZT25, PZT50 and PZT100, as described above. Hereon, the switching for the opposite polarities are defined as Write Up Read Down (WURD) and Write Down Read Up (WDRU).

Figure 4.2 shows the WURD $\triangle \mathrm{Pt} / 2 \mathrm{Ps}$ vs write time switching curves for PZT25, PZT50 and PZT100 and WDRU PZT50. In order to find the relevant switching parameters e.g. characteristic switching times, the dimension of switching and switching activation energy we fitted the above plots with Equations. 1 and 2. Figure 4.3 plot shows the activation field for the four different samples for both biases. It can be seen that for the negative bias the activation field remains almost constant with the $\mathrm{ZnO}$ thickness whereas in the case of the positive polarity the activation field changes by up to 150 times for $100 \mathrm{~nm} \mathrm{ZnO}$. The exponential increase in the effective switching activation field with linear increase in the offset voltage indicates that the nucleation energy is extremely sensitive to the orientation and strength of local dipole near the surface. Since the growth activation field remains unaltered with the polarity of switching we can contend that the increase in the effective activation field is because of the increase in the nucleation switching field. And because of the order of magnitude increase of the nucleation activation field over the growth activation field it allows us to control the switching in the hard direction (higher coercive direction) through nucleation alone. 


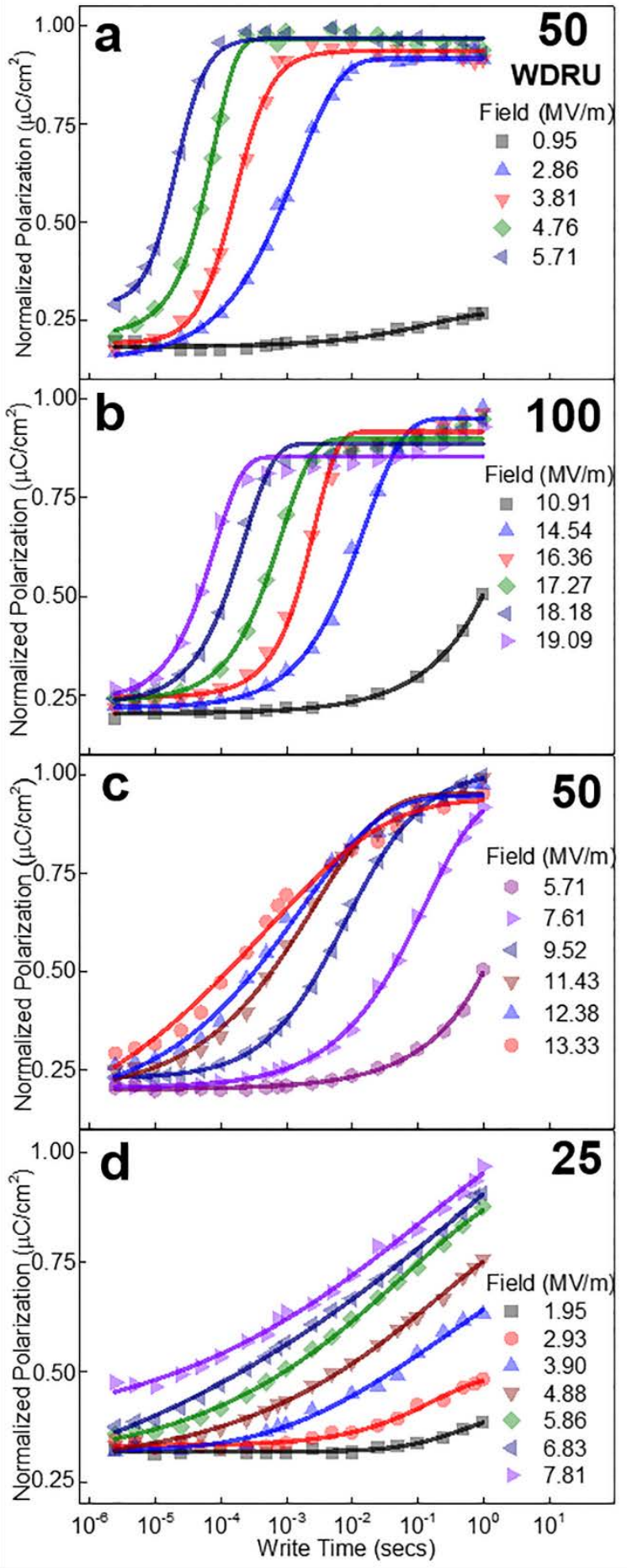

Figure 4.2 Modification of the adaptability of ferroelectric switching with $\mathrm{ZnO}$ thickness Normalized switched polarization measured as a function of write time $\left(t_{3}\right)$ and write field for a) write down read up (WDRU) PZT50, this curve show the switching taking place within an order of magnitude of write time as in KAI model b) write up read down (WURD) PZT100 sample c) write up read down (WURD) PZT50 sample d) write up read down (WURD) PZT25. The normalized switched polarization curves shows a gradual sharpening of the switching with the thickness of $\mathrm{ZnO}$. Measurements were carried out on $200 \mu \mathrm{m}$ by $200 \mu \mathrm{m}$ capacitor structures. The write pulse width (t $\mathrm{t}_{3}$ ) ranging from $2.5 \mu \mathrm{s}$ to $1 \mathrm{~s}$, was applied. The measurements pulses were carried out at a delay time $\mathrm{t}_{1}=1 \mathrm{sec}$. 


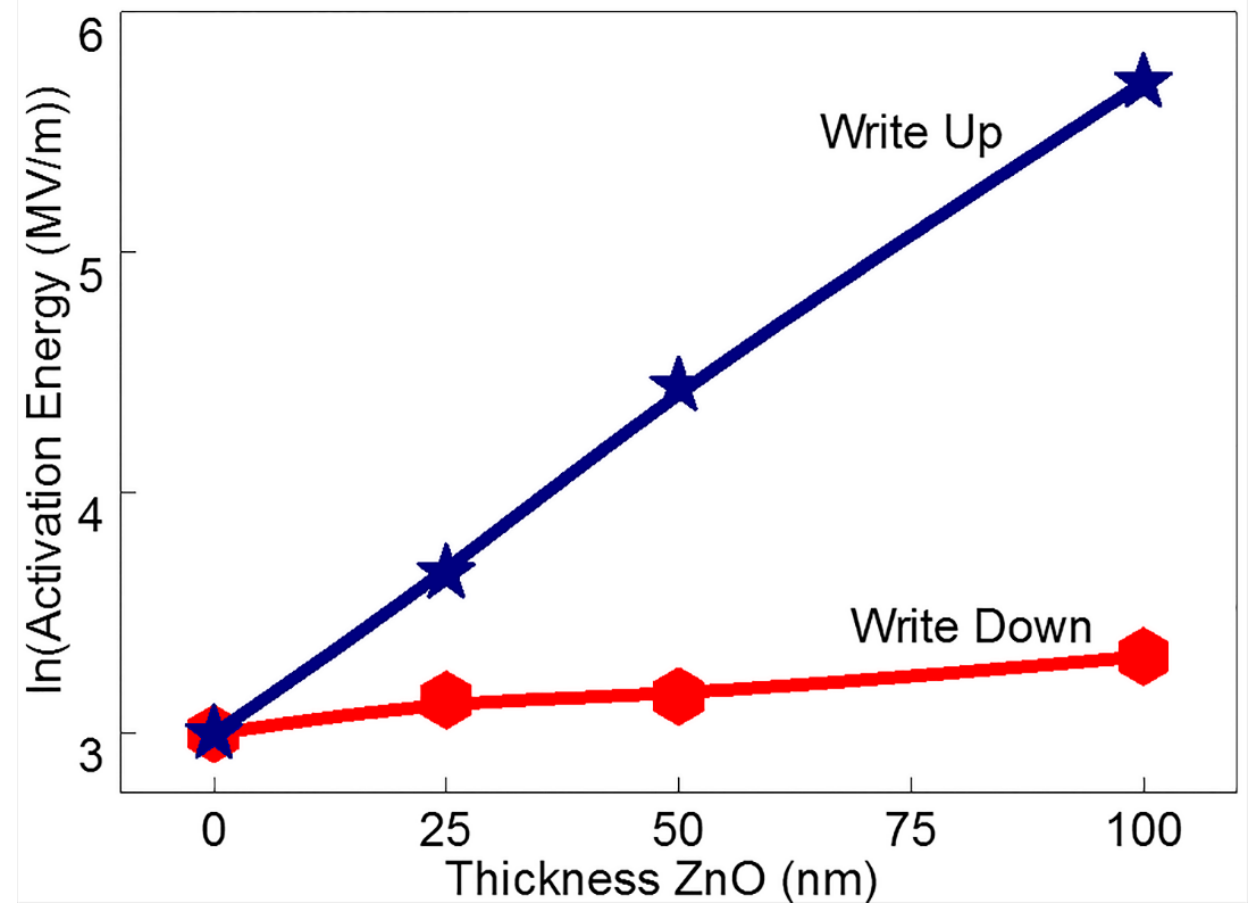

Figure $4.3 \ln$ (Activation field) measured as a function of $\mathrm{ZnO}$ thickness. The curve shows that the activation energy varies exponentially with the $\mathrm{ZnO}$ thickness for the Write Up direction whereas for Write Down it remains almost constant.

We can see that for the WURD in Figure 4.2 that in addition to the shifting of the characteristic growth time to lower values with increase in field there is also an increase in the slope of the curve indicating that switching is governed by a distribution of switching times. For WDRU the shape of the $\Delta \mathrm{P}_{t} / 2 \mathrm{P}_{\mathrm{s}} \mathrm{vs}$ write time switching curves doesn't change with field and is similar to that of PZT indicating that for the negative bias the nucleation is unhindered and the domain coalescence statistics governs the switching. The consistency of the shape of the $\Delta P_{t} / 2 P_{s} v s$ write time switching curves for different fields for PZT and for the WDRU for the other samples points to the uniqueness of the characteristic switching time distribution. In contrast, for WURD it is seen that the sharpness of the switching increases with the thickness of ZnO. Whereas for the PZT and WDRU the sample switches within an order of magnitude of write time, in the case of WURD PZT25 the distribution of switching time spans over six orders of magnitude, for PZT50 over four orders and for PZT100 over 
couple of orders of magnitude. This points to the fact that the distribution of switching times becomes sharper with the thickness of $\mathrm{ZnO}$. The inset of Figure 4.4, shows the effective domain growth dimension of all four samples for the two opposite biases (The inset of Figure $4.4(\mathrm{a}) /(\mathrm{b})$ were obtained by fitting Equation 1 to Figure 4.2). For the WURD we can see that the compared to the WDRU at a given field the domain growth dimensions are much lower. The lower value of dimensionality of switching in the case of WURD arises because of the wide distribution of switching times that becomes narrower with the thickness of $\mathrm{ZnO}$, and is also reflected in the increase of dimensionality with the thickness of ZnO. 

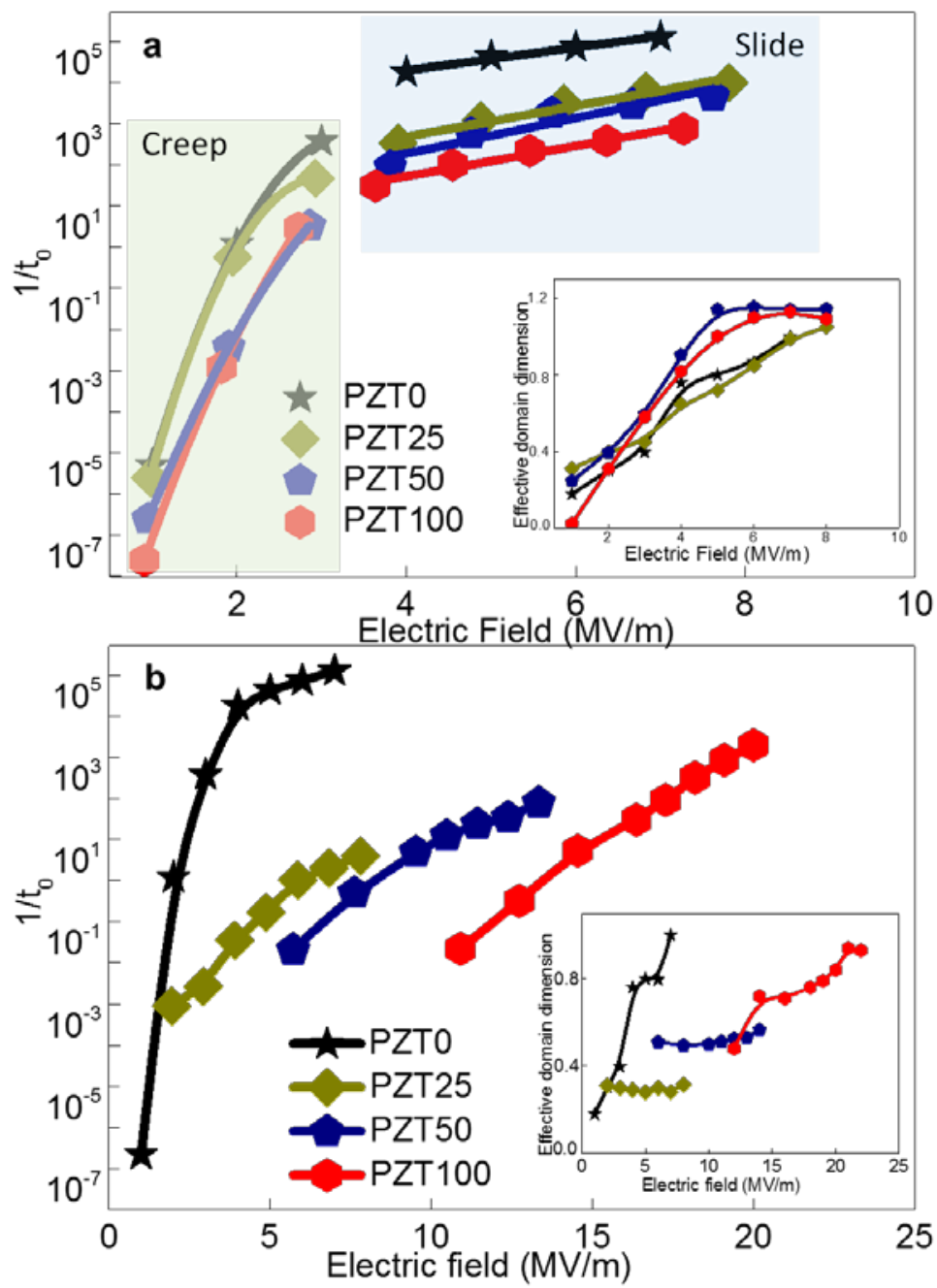

Figure 4.4 Modification of effective domain wall velocity Inverse $t_{0}$ as a function of applied field for different ZnO thicknesses (a) WDRU, (b) WURD. In case of WDRU there is a clear transition from creep to slide motion with increase in electric field but in case of WURD there is no clear distinction between creep and slide motion. (Insets) Effective domain dimension as a function of applied field for different $\mathrm{ZnO}$ thicknesses.

Figure 4.4 shows the inverse of time constant of the domain growth as a function of electric field (E). The velocity of domain growth scales linearly with the inverse of time constant. We 
can see from Figure 4.4 that for WDRU and WURD the domain wall dynamics are markedly different. As a representative comparison we take the example PZTO and PZT50. For WDRU we can see that compared to PZTO the effective domain wall velocity for the same applied electric field $(7 \mathrm{MV} / \mathrm{m})$ is less than a couple of orders of magnitude. In case of WURD the effective domain wall velocity of the PZT50 is about six orders of magnitude lesser than that of PZTO (7 MV/m). For PZT50 the effective domain wall velocities for WURD and WDRU differs by 4 orders of magnitude for the same applied electric field $(7 \mathrm{MV} / \mathrm{m})$. The ferroelectric domain switching can be considered as the growth of an elastic object in a disordered media 42-44. The domain wall velocity has different relationship with the applied field $v=0$ (when pinned at very low electric fields), $\log (v) \alpha E_{\text {ext }}$ (creep at intermediate electric fields), $v \alpha E_{\text {ext }}$ (slide at high electric fields) ${ }^{42-44}$. From Figure 4.4 the slope of $\log \left(1 / \mathrm{t}_{0}\right)$ versus $E$ can give us very important information how the switching is actually taking place. From Figure 4.4 (a) we can see that for WDRU the shape of the curve doesn't change with the thickness of ZnO. From Figure 4.4 (a) we can see that for WDRU the shape of the curve doesn't change with the thickness of $\mathrm{ZnO}$. The domain wall motion is dominated by creep at lower fields and at higher fields is dominated by slide motion. From Figure 4.4 (b) unlike WDRU in WURD there is no sudden change from creep to domain wall slide motion. In case of epitaxial ferroelectric thin films as has been shown by Noh et al ${ }^{44}$ the pinning regime is actually determined by overcoming the initial nucleation barrier, followed by temperature assisted creep and finally slide motion at higher fields. In this case the initial nucleation barrier energy is lower than the onset of the flow regime. In case the nucleation energy is much higher than the onset of the viscous flow regime the domain can in principle directly go into the viscous flow with the suppression of creep regime. However more temperature and frequency dependent studies would be necessary to confirm this.

To analyse the distribution of the switching times and its evolution with the thickness of ZnO the statistics of local electric field distribution at the PZT-ZnO interface was analysed. It is assumed that the ferroelectric film is divided into regions having different switching times.

$$
\Delta \mathrm{P}_{\mathrm{t}}=2 \mathrm{P}_{s} \int_{0}^{\infty}\left[1-\exp \left\{-\left(\frac{t}{t_{0}}\right)^{n}\right\}\right] F\left(\log t_{0}\right) d\left(\log t_{0}\right)
$$


Where $F\left(\log t_{0}\right)$ is the distribution function for $\left(\log t_{0}\right)^{30,31}$. Since the individual switching times are related to the local electric field we need to map the spatial distribution of electric fields. Using the relation $F\left(\log t_{0}\right) d\left(\log t_{0}\right)=F(E) d(E)$ and approximating as a Heaviside step function $\theta\left[E-E_{t h}\right]$ the local reversal of polarization using Equation. 2 and Equation. 1, we can write

$$
\Delta \mathrm{P}_{\mathrm{t}}=2 \mathrm{P}_{s} \int_{0}^{\infty} \theta\left[E-E_{t h}\right] F(E) d(E)
$$

And for statistical normalization

$$
\int_{0}^{\infty} F(E) d(E)=1
$$

Here $E_{t h}$ is the threshold field for a given write time which can be obtained from Equation. 2 and the physical meaning of the above equation is as soon as the applied field exceeds the threshold field polarization switching happens immediately. To get the functional form of the $F(E)$ the derivative of the $\Delta \mathrm{P}_{\mathrm{t}} / 2 \mathrm{P}_{S}$ as a function of the applied electric field was determined for different write times. In order to maintain switching volume conservation the switching curves which reached the saturation polarization within the maximum possible write time of $1 \mathrm{sec}$ were only fitted.

Figure. 4.5 shows the $\Delta \mathrm{P}_{t} / 2 \mathrm{P}_{s}$ as a function of the applied electric field for different write times for the PZT50 sample for WDRU (The plots for PZT and WDRU of PZT25 as well as PZT100 have similar characteristics.). Since the data points were scattered the curves which are shown here were spline fitted. As can be seen from Figure. 4.5 (a) as the write times decrease the maximum peak voltage increases. The observed plots were found to fit best with Lorentzian distribution functions as compared to a Gaussian. Figure. 4.5 (b) shows the rescaled plots of Figure. 4.5 (b) using $\left(E-E_{\max }\right) / \mathrm{w}$ where $E_{\max }$ is the central maximum value and $w$ is the full width at half maxima. This scaling behaviour suggests that the distribution is intrinsic. 


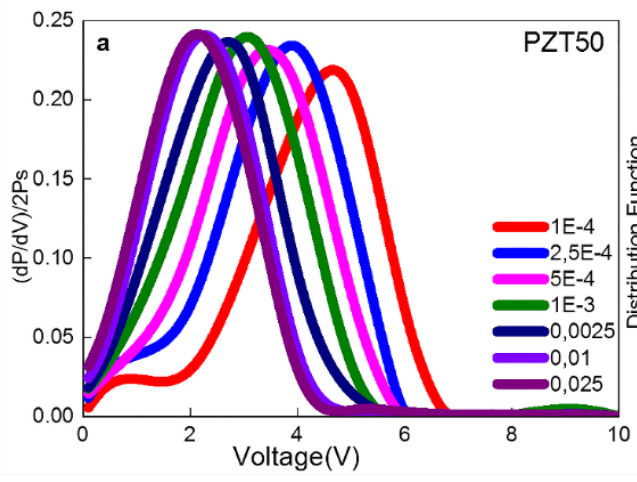

(a)

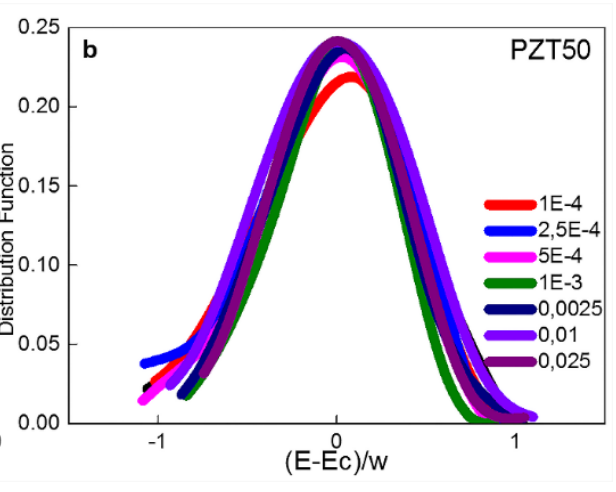

(b)

Figure. 4.5 (a) Logarithmic derivative of the fractional switched polarization versus applied voltage (b) Normalized distribution of activation field for WDRU for PZT50. All the distributions were found to fit best with a Lorentzian.

Similarly as above, Figure. 4.6 shows the $\Delta \mathrm{P}_{t} / 2 \mathrm{P}_{s}$ as a function of the applied electric field for different write times for the PZT50 sample for WURD. Similar to as observed in Figure. 4.5 (a) as the write times decrease the maximum peak voltage increases. As we can see from Figure. 4.6 (a) the curves don't quite follow any regular shape and also the shape varies with the write time. It signifies that the local fields are not symmetrically distributed about the mean. Since the switching here is governed by the local field variations at the ZnO-PZT interface, which is dependent on the roughness and local disorder the distribution of local electric fields need not necessarily be a singular well defined peak function. In order to rescale these asymmetric curves the asymmetric double sigmoid function was used. The switching curves with the asymmetric double sigmoid (ASD) curve was fitted.

$y=B^{*}\left(1 /\left(1+e^{(-(E-E \operatorname{Emax}+w 1 / 2) / w 2))}\right) *\left(1-1 /\left(1+e^{(-(E-E \operatorname{Emax}-w 1 / 2) / w 3))}\right)\right.\right.$

Its notable here that Tagantsev et al assumed a mesa like function to map the distribution of characteristic switching times which can qualitatively describe the linear $\Delta \mathrm{P}_{t} / 2 \mathrm{P}_{s}$ vs write time curve but would not be sufficient to fully map the local electric fields as in our case. As in Equation. $6 E_{\max }$ is the central value of the derivative and $w_{1}$ is the full width at the half maxima and $w_{2}$ and $w_{3}$ determines the asymmetry of the distribution in the lower and higher value than $E_{\max }$. The above curves was rescaled using $\left(E-E_{\max }\right) / w_{1}$ which is shown in Figure. $4.6(b)$. 


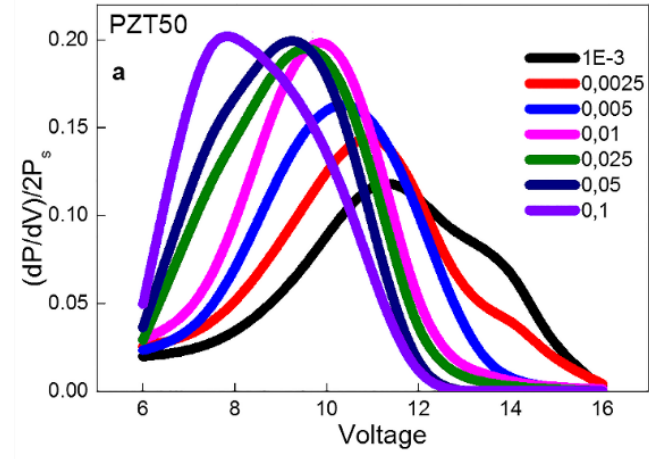

(a)

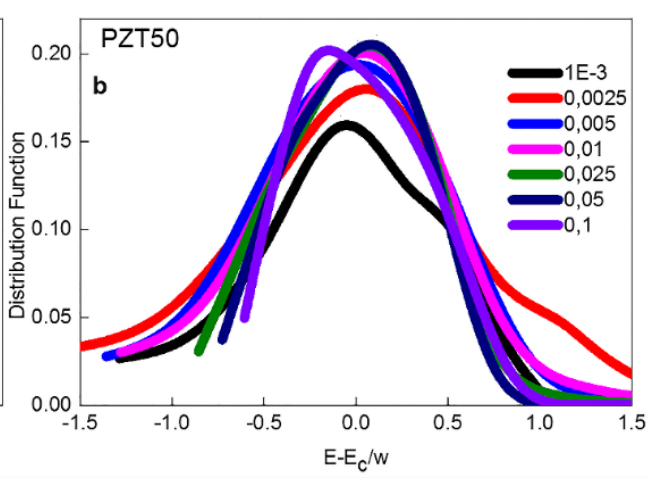

(b)

Figure. 4.6 (a) Logarithmic derivative of the fractional switched polarization versus applied voltage (b) Normalized distribution of activation field for WURD for PZT50. All the distributions were found to fit best with an asymmetric double sigmoidal function.
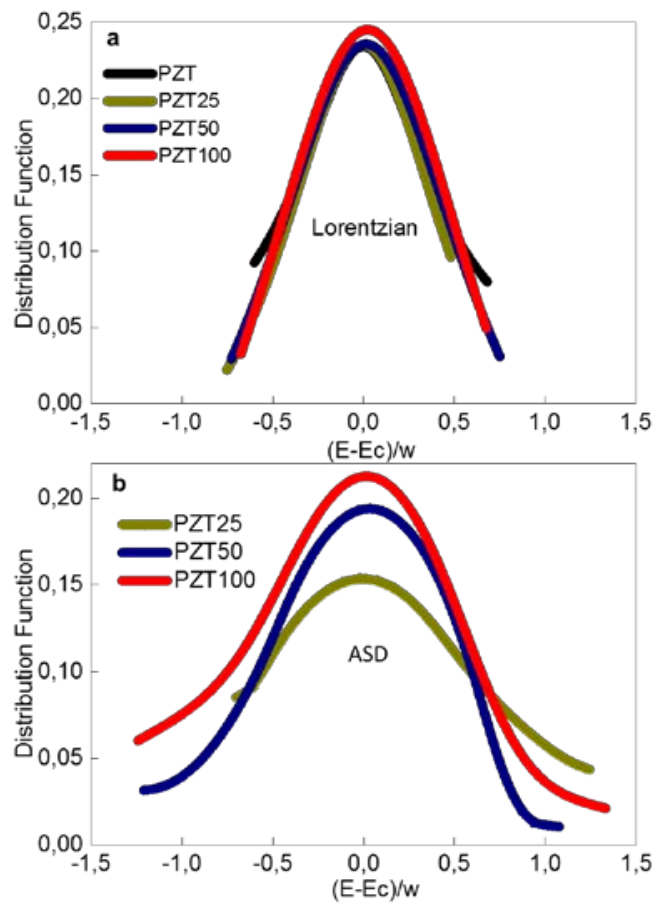

Figure 4.7 Distribution of local nucleation field a) Normalized distribution of activation field for WDRU for PZT, PZT25, PZT50 and PZT100. All the distributions were found to fit best with a Lorentzian. The distribution activation field of all the films are same. b) Normalized distribution of activation field for WURD for PZT25, PZT50 and PZT100. All the distributions have been normalized with an asymmetric double sigmoidal function. The distribution of the activation field becomes narrower with increase in ZnO thickness. 
We find that for WDRU and PZT, $F(E)$ fits best with Lorentzian distribution. Normalizing all the derivative of switching curves using $\left(E-E_{\max }\right) / \mathrm{w}{ }^{30,45}$ where $E_{\max }$ is the central value of the derivative and $w$ is the half width at half maxima we see that for all the samples all the curves for different write times merged into a single characteristic curve which shows that the Lorentzian distribution of the activation field is intrinsic. But most importantly we find as shown in Figure 4.7 (a) that the characteristic activation field distribution for $\mathrm{PZT}$, and all the other samples for negative switching direction merge together. It indicates that the total switchable polarization and the distribution of activation fields for all the samples are same which again is consistent with our observation from the switching curves that the polarization switching in normal PZT and the other samples for the lower coercive field direction is governed by the statistics of growth activation field which should remain same for all the samples. A Lorentzian can describe the distribution of horizontal distances at which a line segment tilted at a random angle cuts the $x$-axis. Similarly the distribution of the component along the axis of any fixed electric field at a random angle with the axis is always Lorentzian ${ }^{45}$. In this case if the growth activation field subtends an angle with the growth direction then the distribution of activation field will be a Lorentzian.

For the case of WURD the same procedure as above to find the distribution of the local activation energy was followed.

Unlike the case of the Lorentzian peak fit which can be ab initio ascribed to a well-defined physical phenomena in the case of the WURD the switching is determined entirely by the local field variations, hence one needs a more versatile function which can map these variations. The biggest advantage of the asymmetric double sigmoid (ASD) function is that it can be it can be used to map a very broad distribution of switching time as well as any asymmetry in the distribution function ${ }^{46}$. Furthermore, there is an added advantage pertaining the description of neuron activation function using the ASD function. Since the neuron activation functions are usually sigmoidal (the derivative of a sigmoidal is a double sigmoidal) the distribution of the local electric fields should also be a double sigmoidal in nature to realize the activation functions. Hence analysing the distribution of the local electric fields in terms of an asymmetric double sigmoid can help us correlate the required 
activation function to the actual local electric field distribution. Rescaling the above distribution for the above fitted curves $\left(E-E_{\max }\right) / w_{1}$ as shown in Figure 4.7 (b) unlike Figure 4.7 (a) the curves for PZT25, PZT50 and PZT100 don't merge which shows that the distribution of the local activation nucleation fields are very different from each other. We see that the distribution of activation field becomes sharper with the thickness of ZnO which gives rise to a broader distribution of the switching times which is reflected in the gradual increase of the sharpness of the $\Delta \mathrm{P}_{\mathrm{t}} / 2 \mathrm{P}_{\mathrm{s}}$ switching curves with the thickness of $\mathrm{ZnO}$. Comparing the above distribution of WURD Figure 4.7 (b) with Figure 4.7 (a) it is observed that for the WURD the activation fields are much widely distributed and is also not symmetric as this depends on the roughness of $\mathrm{ZnO}, \mathrm{PZT}$ and also the static disorder at the PZT-ZnO interface. To find out why the distribution of the nucleation activation field becomes sharper with the thickness of $\mathrm{ZnO}$ we note that from Figure 4.3 we can empirically write $\ln \left(E_{a}\right)=$ $A^{*} d_{z n o}$, where $E_{a}$ is the activation energy and $A$ is a constant. Assuming the rms roughness of the $\mathrm{ZnO}$ is $\sigma$. Using the above empirical relation and Equation. 1 and Equation. 2 we can write

$$
E_{a}^{d Z n O+\sigma}=\left(E_{a}^{d Z n O}\right)^{\left(1+\frac{\sigma}{d Z n O}\right)}
$$

Where $E_{a}^{d Z n O+\sigma}$ is the activation field for the thickness dzno+ $\sigma$ and $E_{a}^{d Z n o}$ is the activation energy for $d z n o$. As we can see from the above Equation. 7 the local activation field has a power law relation with the $\frac{\sigma}{d Z n o}$ (normalized roughness), it makes the local activation field extremely sensitive to the normalized roughness of the $\mathrm{ZnO}$ layer. Since the rms roughness of the $\mathrm{ZnO}$ remains almost constant with the thickness of the $\mathrm{ZnO}$ the term $\frac{\sigma}{d Z n o}$ decreases with the thickness of $\mathrm{ZnO}$ which makes the distribution of the activation field sharper with the thickness of $\mathrm{ZnO}$. Here, for simplicity just the roughness of $\mathrm{ZnO}$ was considered, in actual case the roughness of PZT and the static disorder at the PZT-ZnO should also be considered while taking into account $\sigma$. The roughness of PZT and the static disorder at the PZT-ZnO however remains constant with the thickness of $\mathrm{ZnO}$ hence will not have any qualitative difference with our description. This extreme sensitivity of the activation field on the $\frac{\sigma}{d z n o}$ allows us to modify the distribution of the switching times. The value of $\frac{\sigma}{d Z n o}$ can lie between 
0 and 1 and for different values we can achieve different sharpness of the $\Delta \mathrm{P}_{t} / 2 \mathrm{P}_{\mathrm{s}}$ versus write time curve; the value of 0 would correspond to intrinsic switching behaviour. The term $\sigma / d=0$ would mean a perfectly flat and 0 roughness and disorder of the non-switchable ZnO film. On the other hand, in the other extreme of theoretical limit $\sigma / d=1$ would mean the extreme situation of a square wave like pattern which however will make the distinct regions discrete, similarly for a sinusoidal roughness pattern $\sigma / d=1 / \sqrt{ } 2$. As mentioned above the term $\sigma$ represents not only the roughness but the static disorder as a whole. Hence in order to thoroughly model and engineer this particular parameter one would require to take into account not only the roughness of the $\mathrm{ZnO}$ layer but also the shape of the $\mathrm{ZnO}$ as well as the PZT layer at the interface as it would influence the net effective local field.

The most important implication of having a double sigmoidal distribution of local fields (also of distribution of switching time) is in realization of different neuron activation functions. As mentioned earlier in neural networks the activation functions normally used are sigmoidal ${ }^{13,}$ ${ }^{14}$. The very fact that $F(E)$ can be approximated by a double sigmoidal curve would enable us to realize in the switching behaviour of the ferroelectric different activations functions by optimizing the roughness parameters.

From above discussions it can be concluded that by controlling the average roughness and also the shape of the individual regions we can reproduce different activation functions by controlling the local electric fields. This would require to reproducibly create different regions with a substantial control over roughness and shape of the roughness profile. In future, towards achieving this nano-imprint lithography can be a very important technique for creating different regions with different thicknesses. It is possible to create patterns down to $10 \mathrm{~nm}$ in lateral dimensions. It has been known that PZT can be patterned down to 130 $\mathrm{nm}$ in order to achieve high density memory. Additionally, by varying deposition conditions (laser frequency, laser energy, deposition temperature etc) also films with different roughness and shape profiles can fabricated 47.

However, for multi-state memory the ultimate thermodynamic limit for the number of intermediates states will be determined by the Kittel's law. But, even under the limitations of Kittel's law the present day energy storage density (present day device size of $130 \mathrm{~nm}$ ) can 
be increased by 4-5 times for similar perovskite oxide based ferroelectric memories. But in real materials, especially in thin films the stability of a domain can be strongly influenced by the electrode materials and also the presence of defects due to their influence on the depolarizing field. The stability of the states will also be affected on decreasing the device size as the depolarizing fields increase with decrease in film thickness. This restriction imposed by Kittel's law on the number of fractionally switched state will however will not be a limitation for dynamic adaptive operations. More first principles theoretical work as by Rappe et al $^{32}$ on different materials systems taking into consideration different boundary and electrode conditions would indeed be required for a better understanding of the nucleation phenomena.

For realistic applications of ferroelectrics for multi-state memory applications and adaptive applications it is very crucial that the intermediate (fractionally switched) states remain stable with time ${ }^{4}$. It is important that the $\Delta \mathrm{P}_{\mathrm{t}} / 2 \mathrm{P}_{\mathrm{s}} \mathrm{vs}$ write time switching curve shape doesn't change for a given write voltage. Retention failure normally happens due to finite depolarization field, interface traps and presence of electrets ${ }^{21}$. Hence the quality of the interface and the ferroelectric film are of utmost importance. Hence to study the stability of the intermediate states the retention properties of the intermediate states was studied by varying the delay time between write and read to up to 1000 secs. Figure 4.8 shows that the intermediate states for PZT25 at 7 V write voltage are stable up to 1000 secs with very little loss in polarization (within 5\%). For comparison with PZT25 sample it is also shown the retention behaviour of the PZTO sample in Figure 4.8 In general we see that upto 1000 secs the stability of the intermediate states show similar behaviour as the fully switched states. 


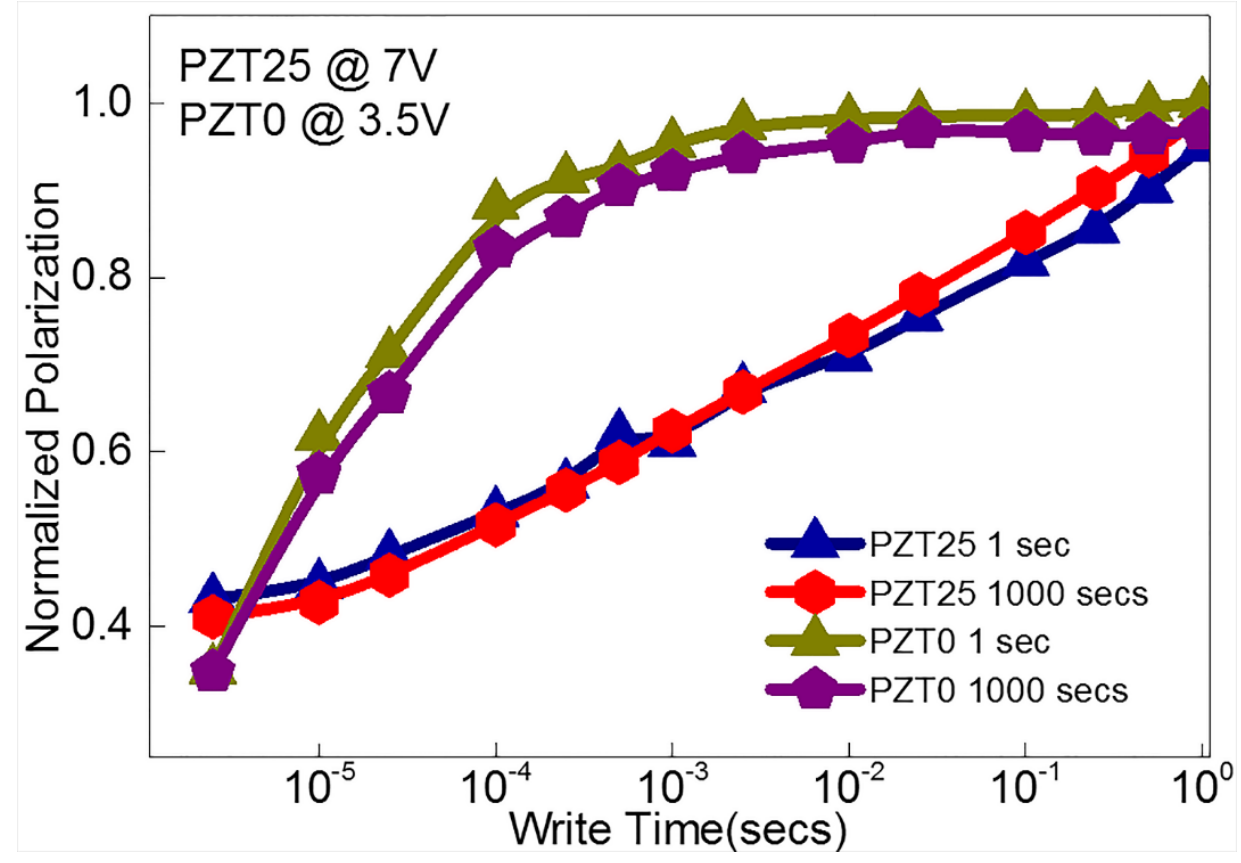

Figure. 4.8 Stability of intermediate states Retention of the intermediate states for PZT25 sample at 7 $\mathrm{MV} / \mathrm{m}$ write field for two different delay times between write and read. Normalized polarization plotted as a function of Write time. The blue curve represents a delay time of $1 \mathrm{sec}$ and the green one a delay of 1000 secs.

The retention of the polarization for all the samples was measured by varying the delay to read time from $10^{-1}-10^{3}$ secs as in Figure. 4.9. The switched polarization was found to be stable with less than a $2 \%$ drop over the measured time scale for PZTO and a maximum of $4 \%$ drop for PZT100 sample. It shows that the switched polarization is stable over time. Here the retention of the switched polarization as a function of delay to read time from $10^{-1}$ to $10^{3}$ secs was plotted for all the samples for both the biases. The retention up to $10^{4}$ secs was studied which showed similar trends as in Figure 4.9. 


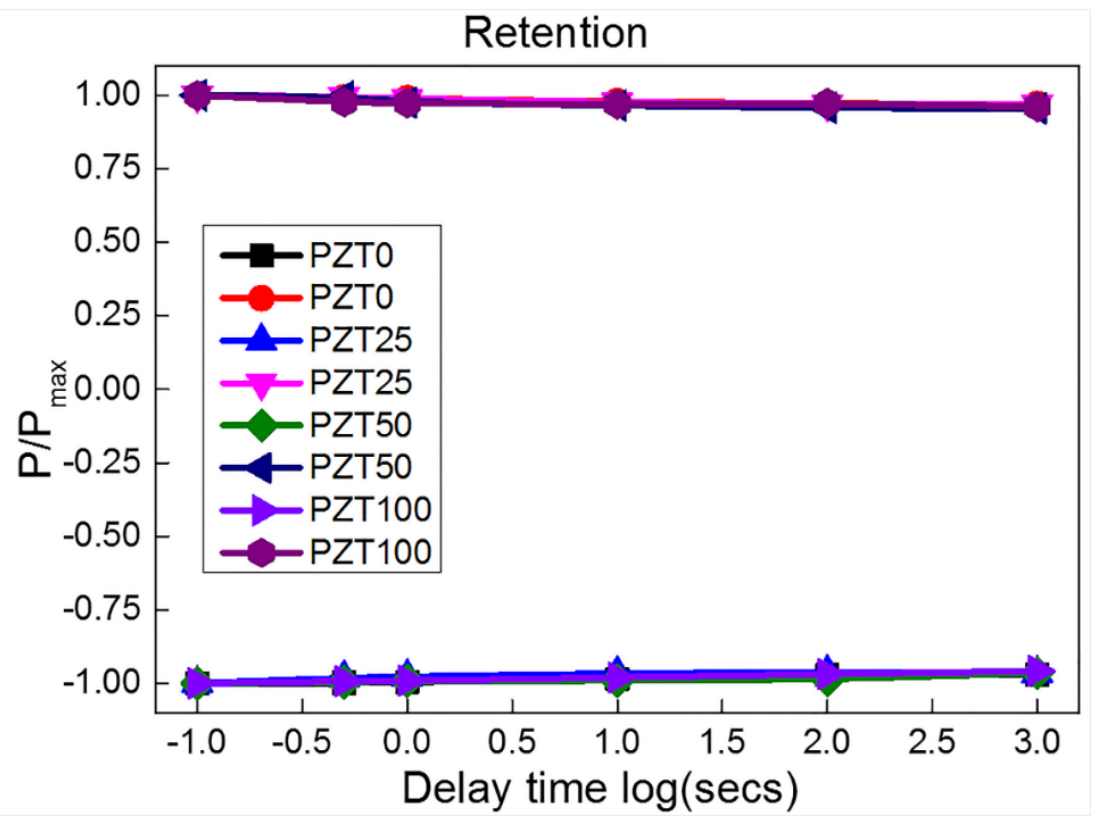

Figure. 4.9 Retention: Switched polarization as a function of delay to read time.

\subsection{Conclusions}

Concluding, it was demonstrated how to create multiple states in an otherwise bi-stable ferroelectric material. The extreme sensitivity of the local switching times on the presence of fixed dipole near the surface allowed us to create independent regions within the same capacitor structure having individual switching times by controlling the relative roughness of the fixed dipole layer. By enabling the ferroelectric to switch in a multivalued manner we not only enable further development in achieving neuron like adaptive memory but also give the possibility to create multi-state memories which can also lead to very high density of storage. These results should stimulate more theoretical and experimental work on methodologies of controlling the switching kinetics in ferroelectrics and other thermodynamically bi-stable materials. 


\subsection{References}

1. Beggs, J. Editorial: Can There Be a Physics of the Brain? Physical Review Letters 114, doi:10.1103/PhysRevLett.114.220001 (2015).

2. André Chanthbouala, Vincent Garcia, Ryan O. Cherifi, Karim Bouzehouane, Stéphane Fusil, Xavier Moya, Stéphane Xavier, Hiroyuki Yamada, Cyrile Deranlot, Neil D. Mathur, Manuel Bibes, Agnès Barthélémy \& Julie Grollier. A ferroelectric memristor. Nature materials 11, 860-864, doi:10.1038/nmat3415 (2012).

3. Fujisaki, Y. Review of Emerging New Solid-State Non-Volatile Memories. Japanese Journal of Applied Physics 52, 040001, doi:10.7567/jjap.52.040001 (2013).

4. Ha, S. D. \& Ramanathan, S. Adaptive oxide electronics: A review. Journal of Applied Physics 110, 071101, doi:10.1063/1.3640806 (2011).

5. Kalinin, S. V., Sumpter, B. G. \& Archibald, R. K. Big-deep-smart data in imaging for guiding materials design. Nature materials 14, 973-980, doi:10.1038/nmat4395 (2015).

6. Ohno, Takeo, Tsuyoshi Hasegawa, Tohru Tsuruoka, Kazuya Terabe, James K. Gimzewski, and Masakazu Aono. Short-term plasticity and long-term potentiation mimicked in single inorganic synapses. Nature materials 10, 591-595, doi:10.1038/nmat3054 (2011).

7. Pershin, Y. V. \& Di Ventra, M. Memory effects in complex materials and nanoscale systems. Advances in Physics 60, 145-227, doi:10.1080/00018732.2010.544961 (2011).

8. Prezioso, Mirko, Farnood Merrikh-Bayat, B. D. Hoskins, G. C. Adam, Konstantin K. Likharev, and Dmitri B. Strukov. Training and operation of an integrated neuromorphic network based on metal-oxide memristors. Nature 521, 61-64, doi:10.1038/nature14441 (2015).

9. Schmidhuber, J. Deep learning in neural networks: an overview. Neural networks : the official journal of the International Neural Network Society 61, 85-117, doi:10.1016/j.neunet.2014.09.003 (2015). 
10. Shi, L. P., K. J. Yi, K. Ramanathan, R. Zhao, N. Ning, D. Ding, and T. C. Chong. Artificial cognitive memory-changing from density driven to functionality driven. Applied Physics A 102, 865-875, doi:10.1007/s00339-011-6297-0 (2011).

11. Dehaene, S., Molko, N., Cohen, L. \& Wilson, A. J. Arithmetic and the brain. Current opinion in neurobiology 14, 218-224, doi:10.1016/j.conb.2004.03.008 (2004).

12. Knight, T., and George Stiny. Classical and non-classical computation Arq: architectural research quarterly 5.04, 355 (2001).

13. Kriesel, D. A brief introduction to neural networks 2011 (August 15 (2007).

14. Zurada, J. M. Introduction to artificial neural systems. St. Paul: West, ( 1992.).

15. Abbott, L. F., and Sacha B. Nelson. Synaptic plasticity: taming the beast Nature neuroscience 3, 1178-1183. (2000).

16. Wang, Zhong Qiang, Hai Yang Xu, Xing Hua Li, Hao Yu, Yi Chun Liu, and Xiao Juan Zhu. Synaptic Learning and Memory Functions Achieved Using Oxygen Ion Migration/Diffusion in an Amorphous InGaZnO Memristor. Advanced Functional Materials 22, 2759-2765, doi:10.1002/adfm.201103148 (2012).

17. Nishitani, Y., Kaneko, Y., Ueda, M., Fujii, E. \& Tsujimura, A. Dynamic Observation of BrainLike Learning in a Ferroelectric Synapse Device. Japanese Journal of Applied Physics 52, 04CE06, doi:10.7567/jjap.52.04ce06 (2013).

18. Jeong, Doo Seok, Reji Thomas, R. S. Katiyar, J. F. Scott, H. Kohlstedt, A. Petraru, and Cheol Seong Hwang. Emerging memories: resistive switching mechanisms and current status. Reports on Progress in Physics 75, 076502 (2012).

19. Strukov, D. B., Snider, G. S., Stewart, D. R. \& Williams, R. S. The missing memristor found. Nature 453, 80-83 (2008). 
20. Ramesh, R. \& Schlom, D. G. Whither oxide electronics? MRS bulletin 33, 1006-1014 (2008).

21. Scott, J. F. Ferroelectric memories. Vol. 3 (Springer Science \& Business Media, 2000).

22. Ishiwara, H. Proposal of adaptive-learning neuron circuits with ferroelectric analogmemory weights. Japanese journal of applied physics 32, 442 (1993).

23. Ishiwara, H. e. a. Ferroelectric neuron circuits with adaptive-learning function. Computers \& electrical engineering 23, 431-438 (1997).

24. Song, S., Kenneth D. Miller, and Larry F. Abbott. Competitive Hebbian learning through spike-timing-dependent synaptic plasticity. Nature neuroscience 3.9, 919-926 (2000).

25. Wen, Z., Li, C., Wu, D., Li, A. \& Ming, N. Ferroelectric-field-effect-enhanced electroresistance in metal/ferroelectric/semiconductor tunnel junctions. Nature materials 12, 617-621, doi:10.1038/nmat3649 (2013).

26. Mathews, S., Ramesh, R., Venkatesan, T. \& Benedetto, J. Ferroelectric field effect transistor based on epitaxial perovskite heterostructures. Science 276, 238-240 (1997).

27. Ishibashi, Y., and Yutaka Takagi. Note on ferroelectric domain switching. Journal of the Physical Society of Japan 31, 506-510 (1971).

28. Tagantsev, A. K., Cross, L. E. \& Fousek, J. Domains in ferroic crystals and thin films. (Springer, 2010).

29. Tagantsev, A. K., Stolichnov, I., Setter, N., Cross, J. S. \& Tsukada, M. Non-KolmogorovAvrami switching kinetics in ferroelectric thin films. Physical Review B 66, doi:10.1103/PhysRevB.66.214109 (2002).

30. Jo, J. Y., H. S. Han, J-G. Yoon, T. K. Song, S-H. Kim, and T. W. Noh. Domain switching kinetics in disordered ferroelectric thin films. Phys Rev Lett 99, 267602, doi:10.1103/PhysRevLett.99.267602 (2007). 
31. Zhukov, S., Genenko, Y. A. \& von Seggern, H. Experimental and theoretical investigation on polarization reversal in unfatigued lead-zirconate-titanate ceramic. Journal of Applied Physics 108, 014106, doi:10.1063/1.3380844 (2010).

32. Shin, Y. H., Grinberg, I., Chen, I. W. \& Rappe, A. M. Nucleation and growth mechanism of ferroelectric domain-wall motion. Nature 449, 881-884, doi:10.1038/nature06165 (2007).

33. Kim, Yunseok, Hee Han, Woo Lee, Sunggi Baik, Dietrich Hesse, and Marin Alexe. NonKolmogorov-Avrami-Ishibashi switching dynamics in nanoscale ferroelectric capacitors. Nano letters 10, 1266-1270, doi:10.1021/n19038339 (2010).

34. Gerra, G., Tagantsev, A. K. \& Setter, N. Surface-Stimulated Nucleation of Reverse Domains in Ferroelectrics. Physical Review Letters 94, doi:10.1103/PhysRevLett.94.107602 (2005).

35. Stephen Jesse, Brian J. Rodriguez, Samrat Choudhury, Arthur P. Baddorf, Ionela Vrejoiu, Dietrich Hesse, Marin Alexe, Eugene A. Eliseev, Anna N. Morozovska, Jingxian Zhang, LongQing Chen \& Sergei V. Kalinin. Direct imaging of the spatial and energy distribution of nucleation centres in ferroelectric materials. Nature materials 7, 209-215, doi:10.1038/nmat2114 (2008).

36. Haun, M., Furman, E., Jang, S. \& Cross, L. Thermodynamic theory of the lead zirconatetitanate solid solution system, part I: phenomenology. Ferroelectrics 99, 13-25 (1989).

37. Janotti, A. \& Van de Walle, C. G. Fundamentals of zinc oxide as a semiconductor. Reports on Progress in Physics 72, 126501, doi:10.1088/0034-4885/72/12/126501 (2009).

38. Tagantsev, A. K. \& Gerra, G. Interface-induced phenomena in polarization response of ferroelectric thin films. Journal of Applied Physics 100, 051607, doi:10.1063/1.2337009 (2006).

39. Anirban Ghosh, Gertjan Koster \& Guus Rijnders Tunable and temporally stable ferroelectric imprint through polarization coupling. APL Mater. 4, 066103 (2016). 
40. K. Abe, N. Yanase, T. Yasumoto and T. Kawakubo, Nonswitching Layer Model for Voltage Shift Phenomena in Heteroepitaxial Barium Titanate Thin Films. Japanese Journal of Applied Physics 41 (Part 1, No. 10), 6065-6071 (2002).

41. Li, W. \& Alexe, M. Investigation on switching kinetics in epitaxial $\mathrm{Pb}(\mathrm{ZrO} 0.2 \mathrm{TiO} 0.8) \mathrm{O} 3$ ferroelectric thin films: Role of the $90^{\circ}$ domain walls. Applied Physics Letters 91, 262903, doi:10.1063/1.2825414 (2007).

42. Giamarchi, T., Kolton, A. \& Rosso, A. in Jamming, Yielding, and Irreversible Deformation in Condensed Matter 91-108 (Springer, 2006).

43. Hu, Wei Jin, Deng-Ming Juo, Lu You, Junling Wang, Yi-Chun Chen, Ying-Hao Chu, and Tom Wu. Universal ferroelectric switching dynamics of vinylidene fluoride-trifluoroethylene copolymer films. Scientific reports 4, 4772, doi:10.1038/srep04772 (2014).

44. Yang, Sang Mo, Ji Young Jo, T. H. Kim, J-G. Yoon, T. K. Song, Ho Nyung Lee, Zsolt Marton, S. Park, Y. Jo, and Tae Won Noh. ac dynamics of ferroelectric domains from an investigation of the frequency dependence of hysteresis loops. Physical Review B 82, doi:10.1103/PhysRevB.82.174125 (2010).

45. Weisstein, E. W. Cauchy Distribution, http://mathworld.wolfram.com/CauchyDistribution.html

46. Di Marco, V. B. \& Bombi, G. G. Mathematical functions for the representation of chromatographic peaks. Journal of Chromatography A 931, 1-30 (2001).

47. R. Eason (Ed.), Pulsed Laser Deposition of Thin Films (pp. 177-190). New Jersey, USA: John Wiley \& Sons, Inc..(2006)

48. Nguyen, M. D M. Dekkers, E. Houwman, R. Steenwelle, X. Wan, A. Roelofs, T. SchmitzKempen and G. Rijnders,. Misfit strain dependence of ferroelectric and piezoelectric properties of clamped (001) epitaxial $\mathrm{Pb}\left(\mathrm{Zr}_{0.52}, \mathrm{Ti}_{0.48}\right) \mathrm{O}_{3}$ thin films. Applied Physics Letters 99, 252904 (2011). 
49. M. Grossmann, O. Lohse, D. Bolten, U. Boettger, T. Schneller and R. Waser, Journal of Applied Physics 92 (5), 2680 (2002) 


\section{CHAPTER 5 \\ ELECTRICAL CHARACTERIZATIONS OF PZT-ZNO HETEROSTRUCTURES}

The semiconducting nature of the ferroelectric PZT were investigated in this chapter. Temperature dependent leakage (I-V) were performed on SRO/PZT/ZnO/SRO heterostructures to understand the leakage mechanism acting in these heterostructures. The leakage mechanism was found to be due to interface controlled Schottky emission and was analysed using the Schottky Simmons equation. The built-in voltage at different interfaces (SRO-PZT and PZT-ZnO) and effective charge densities were determined. Temperature dependent capacitance voltage $(\mathrm{C}-\mathrm{V})$ measurements was used to determine the free charge carrier density inside the PZT thin films. The interface trap density was determined using the parallel conductance method and was found to be of the order of $10^{10} \mathrm{~cm}^{-2}$. At low voltages negative differential resistivity regime was observed and was ascribed to trap emptying and filling. Admittance measurements were performed which showed that capacitive contribution was much higher than the leakage contribution. 


\subsection{Introduction}

Electronic devices based on ferroelectric MFM structures depend on reading currents due to polarization changes under the application of an external voltage ${ }^{1-3}$. In the ideal limit ferroelectrics for this purpose are assumed to be ideal insulators and the response of the MFM structures totally capacitive in nature. In bulk ferroelectrics, both single crystal and ceramics, the above presumption can be quite good, as the leakage contribution is orders of magnitude lower than the capacitive contribution ${ }^{4}$. However, for thin film MFM structures the leakage contributions can be quite significant to obscure the capacitive contribution due to polarization differential. A significant amount of leakage can adversely affect the ferroelectric hysteresis loop, which is obtained by integrating the charge released during polarization switching 1,567. Additionally, the presence of free charge carriers also has a detrimental effect on the reliability of the devices including fatigue, retention etc. 1, 8-11. Hence, it becomes utmost important to understand the leakage mechanism in MFM/MFSM structures so that these detrimental effects can be minimized.

Regarding the transport properties an MFM structure can be considered as two back to back Schottky diodes arising from the interface between a metal and a semiconductor 12-16. Ferroelectric undoped PZT is a wide band gap semiconductor and is normally considered as a p-type semiconductor in bulk because of its defect chemistry 1,17-19. However in some literature due to the higher mobility of electrons and due to oxygen vacancies at the surface PZT has also been considered as n-type semiconductors 1,20 .

Nevertheless, oxide ferroelectrics like PZT cannot be treated as any normal semiconductor because of the extreme low mobility of the free charge carriers due to very small mean free paths as well as the very long relaxation times ${ }^{13,21}$. This also introduces serious challenges in their characterization using conventional semiconductor characterization methods. ZnO on the other hand is normally an $n$-type semiconductor due to the $\mathrm{O}$ vacancies ${ }^{22,23}$. Sincea ferroelectric can be considered both an insulator and a semiconductor, a comprehensive understanding of an MFM or MFS structure can only be obtained by P-E, C-V and I-V measurements together $1,12,13,24$. In the previous chapters the polarization-electric field characterizations were performed in detail, here we consider the $\mathrm{C}-\mathrm{V}$ and I-V measurements 
to understand the semiconducting character of ferroelectrics. I-V measurements are used to determine the built-in voltage at the two heterojunctions as well as the main leakage mechanism in the studied heterostructures. C-V measurements can be used to determine the free carrier density inside the PZT film as well as to find out the interface trap density at the ferroelectric-electrode interface ${ }^{12,13,25,26 .}$

\subsection{Transport Mechanisms and Current-Voltage (IV) Measurements}

As mentioned above for transport properties an MFM structure can be considered as two back to back Schottky diodes connected via a resistor. Hence, the conduction mechanism in these structures can be governed by either the bulk resistance or the interface resistance ${ }^{2-}$ 4,27. Bulk limited mechanisms can be categorized as a) Ohmic conduction, b) Space charge limited currents (SCLC), c) Poole - Frenkel emission (PFE), d) Hopping conduction, e) Ionic conduction, and f) Grain boundary limited conduction 1, 3,4. Interface limited conduction can be divided into a) Thermionic or Schottky emission (SE), b) Fowler-Nordheim tunnelling (FNT), and c) Direct tunnelling 1,3,4. Even though one of the above mechanisms may be the main current limiting one, all or more than one of the above mechanisms may be operating simultaneously and are not completely independent from one another ${ }^{1,} 27$. Moreover, different conduction mechanisms may be acting at different voltage regimes. Hence, it becomes an experimental challenge to determine the main operating mechanisms responsible for leakage. Below we briefly describe all the conduction mechanisms in detail and after that we describe how different I-V and C-V measurements can be performed to determine the relevant parameters. 


\subsubsection{Bulk Limited Conduction Mechanisms}

The bulk-limited conduction mechanisms depend on the electrical properties of the ferroelectric itself.

a) Ohmic conduction:

Ohmic conduction is caused by the mobile electrons in the conduction band and holes in the valence band. In this conduction mechanism, a linear relationship exists between the current density and the electric field and is symmetric with respect to electric field. Even though the band gap of ferroelectrics is quite wide, there will still be a finite amount of charge carriers contributing to Ohmic conduction due to thermal excitation. It is normally observed at low electric fields in oxide insulators. The current density in this case is expressed as

$$
J_{o h m}=\left(n_{e} \mu_{e}+n_{h} \mu_{h}\right) q E
$$

where, $n_{e}, n_{h}$ is the number of free electrons and holes, q elementary charge, and $\mu$ the mobility ${ }^{28}{ }^{3}$ and $E$ is the applied electric field.

b) Space charge limited current (SCLC):

Space-charge-limited conduction (SCLC) is similar to the transport of electrons in a vacuum diode due to injected space charge inside the ferroelectric 1,3,29,30. The current densityvoltage characteristic of a vacuum diode is governed by Child's law:

$$
J_{S C L C}=9 / 8 \mu \varepsilon_{0} \varepsilon_{r} E^{2} / d^{3}
$$

where, $\mu$ the mobility and $d$ the film thickness (in the above equation it is assumed that the mobility remains unaffected by the electric field), and $\varepsilon_{r}$ is the static dielectric constant. Since SCLC is directly proportional to $d^{-3}$ the thickness of the film, thickness dependence study of $\mathrm{I}-\mathrm{V}$ characteristics are necessary to determine their contribution especially for very thin films. We also study thickness dependent I-V behaviour to determine the contribution of space charge to the leakage contribution ${ }^{1}$.

c) Poole - Frenkel emission (PFE):

Poole-Frenkel emission is very similar to Schottky emission; namely, the thermal excitation induced electron emission from traps into the conduction band of the dielectric. Considering an electron inside a trapping centre, the Coulomb potential energy of the electron can be 
reduced by an applied electric field across the dielectric film. This reduction in potential energy will increase the probability of an electron being thermally excited out of the trap into the conduction band. The current density in this case is expressed as

$$
J_{P F E}=n q \mu E \exp \left[-q\left\{\varphi_{T}-\left(q E / \pi \varepsilon_{o p}\right)^{1 / 2}\right\} / k T\right]
$$

where, $\phi_{T}$, is the trap energy and $\varepsilon_{o p}$ is the optical permittivity. Even though PFE current density vs electric field has similar characteristics as Schottky emission it can be distinguished from the later due to its symmetric nature with respect to applied electric field $1,3,27,31$.

d) Hopping conduction:

Hopping conduction is from the tunnelling effect of trapped electrons hopping from one trap site to another in ferroelectric films. Unlike thermally activated electron emission in PFE it is determined by the applied electric field. Due to the asymmetric and temperature dependent characteristics of the measured I-V we don't consider the contribution of this mechanism in our films 3,3233 .

e) Ionic conduction:

Ionic conduction originates from the movement of ions under an applied electric field. The formation of the ions come due to the presence of lattice defects in the dielectric films. However because of the heavy ionic mass, this mechanism is usually not important apropos ferroelectric films in CMOS technology. Hence in this chapter we neglect the contribution of ionic conduction ${ }^{3} 28$.

f) Grain boundary limited conduction:

In a polycrystalline ferroelectric material, the resistivity of the grain boundaries may be much different than that of the grains. In this case, the conduction current could be limited by the electrical properties of the grain boundaries. In our case the films are epitaxial hence we don't consider grain boundary conduction in our analysis of the I-V measurements $3,28$. 


\subsubsection{Interface Limited Conduction}

The interface limited conduction mechanisms depend on the electrical properties of the electrode-ferroelectric contact. The most important parameter is the barrier height at the electrode-ferroelectric interface.

a) Thermionic or Schottky emission:

A ferroelectric metal contact can be considered as a Schottky contact. When a metal and semiconductor are brought into contact, electrons/holes flow from the semiconductors into the metal until the Fermi energies (more accurately in the case of semiconductors it should be called the electrochemical potential) of both sides are equal ${ }^{1,34-37}$. This redistribution of charges leads to a band bending as shown in Figure 5.1

a)

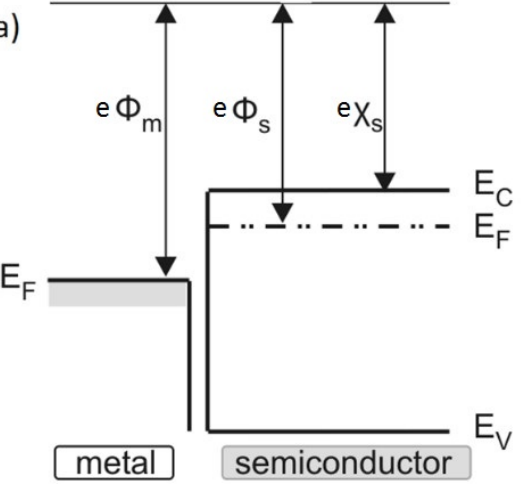

b)

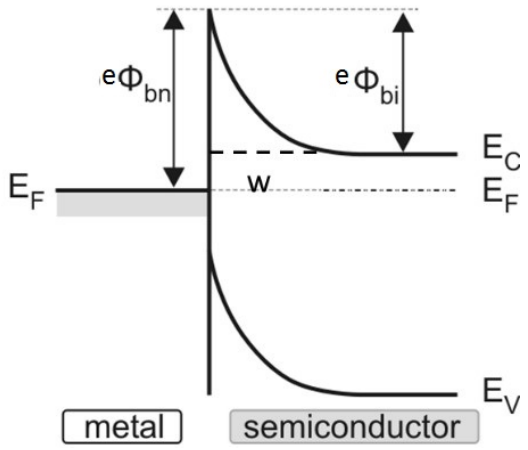

Figure 5.1 Band alignment diagram between a metal and a semiconducto a) when not in contact b) when in contact.

For an $n$-type semiconductor the contact is Ohmic if $\phi_{M}<\phi_{S}$, and rectifying if $\phi_{M}>\phi_{S}{ }^{1}$. The band bending in the ideal case can be quantified as

$$
\varphi^{O_{B}}=\varphi_{M}-\chi_{S}
$$

where, $\phi_{\mathrm{s}}, \phi_{\mathrm{M}}$, and $\chi_{\mathrm{s}}$ are the semiconductor work function, metal work function and semiconductor electron affinity respectively. In the above diagram $\mathrm{E}_{\mathrm{C}}, \mathrm{Ev}_{\mathrm{v}}$ and $\mathrm{E}_{\mathrm{F}}$ stands for the conduction band, valence band and Fermi level energy. In Figure 5.1(b), w represents the depletion layer thickness. 
The above equation doesn't take into account the role of interface states, surface states (broken bonds at the surface) and only considers the bulk electron energy levels. However in practice at the surface the bonds are broken which can change the electron energy levels, and unlike inside the bulk the surface can also be charged which will change the charge neutrality level 373814 . Depending upon the type of surface charge the surface can act as either an acceptor or donor type dopant. Moreover, in any heterostructure interface surface states are also present which can also alter the Fermi energy (electrochemical potential) of the semiconductor. In case the surface state density is very high the actual band bending (built-in potential) will be governed by the surface state energy level ${ }^{15,16}$. Depending upon the type of these surface states the energy level can be either close to the valence or the conduction band, which can in effect lead to a reduced band bending. The limit when the bend bending is completely determined by the interface states, also known as the Bardeen limit, which can be expressed as

$$
\varphi^{\varrho_{B}}=S\left[\varphi_{M}-\chi S\right]+(1-S)\left[E_{g}-\varphi_{0}\right]
$$

where, $E_{g}$ is the semiconductor band gap and $\phi_{0}$ the charge neutrality level at the surface ${ }^{37-}$ ${ }^{41}$. As can be seen from the above equation $S=1$, corresponds to the Schottky limit, and $S=$ 0 corresponds to the Bardeen limit.

In ferroelectrics the presence of polarization charges adds to additional complexities within the present Schottky diode concept of the MFM structure. The switchable polarization charges can be visualized as a sheet of surface charge at the ferroelectric metal interface ${ }^{4}$ 12. Depending upon the nature of this sheet charge it can increase or decrease the built-in voltage. Moreover due to the presence of switchable polarization care should be taken to eliminate the contribution of polarization charges in the leakage current.

The current density when controlled by interface limited Schottky emission can be expressed as

$$
J_{S C}=A^{*} T^{2}\left[-q\left\{\varphi^{O}-\left(q E / 4 \pi \varepsilon_{0} \varepsilon_{r}\right)^{1 / 2}\right\} / k T\right]
$$

where, $A^{*}$ is the effective Richardson coefficient (here we use the term effective since it also depends on the effective mass of the electron inside the ferroelectric film) ${ }^{3}$. As can be seen 
from the above equation this current decreases exponentially with the band bending and increases exponentially with the temperature.

As can be understood from the above discussions the built-in voltage $\left(V_{b i}\right)$, effective space charge density $\left(\mathrm{N}_{\mathrm{eff}}\right)$ and the depletion layer width $(\mathrm{w})$ are the important parameters for a Schottky diode and which can be determined from the temperature dependent I-V measurements 12,1342 .

$$
\begin{gathered}
V_{b i}=\varphi^{O_{B}}-k T / q \ln \left[N_{\text {eff }} / N_{d o p}\right] \\
w=\left[2 \varepsilon_{o} \varepsilon_{r}\left(V+V_{b i}\right) / q N_{e f f}\right]^{1 / 2}
\end{gathered}
$$

In the above equation it was assumed that the mean free path of the charge carriers is much larger than the depletion width. As mentioned earlier oxide ferroelectrics cannot be considered as normal semiconductors due to the extremely short mean free path of the electrons and the above equation will get modified. Simmons derived the correct equation for that case which is commonly known as the Schottky-Simmons equation 1, 27, 43, 44.

$$
J_{S S}=\alpha \mu E\left(m^{*} / m_{0}\right)^{3 / 2} T^{3 / 2}\left[-q\left\{\varphi^{0}-\left(q E / 4 \pi \varepsilon_{0} \varepsilon_{r}\right)^{1 / 2}\right\} / k T\right]
$$

Here, $\mathrm{m}^{*}$ is the effective electron/hole mass inside the ferroelectric, mo is the free electron mass, $\mu$ is the electron mobility and $\alpha=3^{*} 10^{-4} \mathrm{Ascm}^{3} / \mathrm{K}^{3 / 2}$. As can be seen due to the pre exponential linear factor of $E$ at low voltages (when the exponential term is very low) the ferroelectric will behave like an Ohmic conductor. Temperature dependent I-V measurement can be performed to determine $\phi^{0}$ в and will be discussed later in the section 4.6.1 4527,46 .

b) Fowler-Nordheim tunnelling:

As was mentioned in the previous section a metal ferroelectric interface forms a potential barrier due to band bending. Fowler-Nordheim (F-N) tunnelling occurs when the applied electric field is large enough so that the electron wave function penetrates through the triangular potential barrier into the conduction band of the ferroelectric. The FN tunnelling current can be expressed as

$$
J_{F N}=A E^{2} \exp [-B / E]
$$


where, $A$ is $1.38072 * 10^{-6} / \phi A m / \mathrm{eV}^{2}$, and $B$ is $6.3995^{*} \phi^{3 / 2} \mathrm{eV} / \mathrm{nm}$. As can be seen from the above equation this mechanism is temperature independent and hence temperature dependent I-V measurements can be performed to examine the role of this mechanism ${ }^{1,3}$, 4748 .

c) Direct tunnelling:

For very thin oxide films if the electric field across the oxide can be too high that electrons can tunnel through the oxide from one electrode to the other. Unlike FN tunnelling where the tunnelling is only across the interface layer in this case the tunnelling occurs across the whole oxide. The current here decays exponentially with the $\left(\phi_{B}\right)^{1 / 2}\left(\phi_{B}\right.$ barrier height) and the thickness of the oxide layer $3,49,50$. This concept has recently been applied to make ferroelectric tunnel junctions (FTJ) wherein the barrier height was modified by switching the polarization. In our case the thickness of the films is $\sim 1 \mu \mathrm{m}$ thus we can neglect this mechanism as possible conduction mechanism.

\subsection{Capacitance-Voltage Measurements}

C-V measurements have been one of the most basic and important characterization techniques especially for metal-oxide-semiconductor (MOS) structures. Unlike P-E and I-V measurements, C-V being small signal quasi-static in nature allows one to examine small fluctuations in the space charge region ${ }^{25,51}$. C-V measurements are used to determine the doping concentration, doping profile, built-in potential, flat band voltage, oxide capacitance (for MOS structures), density of interface states and the charge density in the oxide layer 1 , 42,52. However, unlike in MOS structures, in MFM/MFS structures, due to the switchable polarization of the ferroelectric, measurements can often be dominated by the movement of the polarization charges within the coercive voltage regime. Hence for our measurements we consider the $\mathrm{C}-\mathrm{V}$ curves only at high voltages where the contribution from polarization switching becomes very small. Moreover frequency and temperature dependence $\mathrm{C}-\mathrm{V}$ measurements can give us a lot of insight into the defect/trap dynamics which is especially important for heterostructures. Here, we have used C-V measurements to study the free 
carrier density inside the ferroelectric film and their temperature dependence and the interface trap density. Below we describe each one of them briefly $13,24,53,54$.

\subsubsection{Free carrier density}

Within the Schottky diode picture of an MOS structure the doping density can be written as $12,13,24,25,42,53,54$

$$
N_{d o p}=2 / q \varepsilon_{0} \varepsilon_{r}\left[d\left(1 / C^{2}\right) / d V\right]
$$

The above equation assumes uniform doping in the space charge region. In the above equation $\mathrm{N}_{\text {dop }}$ represents only the mobile charge carrier density not the effective charge inside the space charge region, since only the mobile charges can respond to the small high frequency ac signal. Due to the presence of switchable polarization only at higher voltage the value of $d\left(1 / C^{2}\right) / d V$ should be taken into account for proper calculation of $N_{d o p}$. In the above equation the low frequency/static permittivity $\varepsilon_{r}$ is determined from the saturated part of the P-E hysteresis loop. Temperature dependence studies can also shed light into the ionization of traps in the bulk. With increase of temperature the deep trap levels will get ionized and will significantly affect the $\mathrm{C}-\mathrm{V}$ characteristic. If in case the carrier concentration doesn't change with temperature it can be inferred that the energy levels of the free carriers are shallow and the film is completely ionized at room temperature 13,55 .

\subsubsection{Interface trap density}

The presence of traps in an MFM/MFS structure can significantly affect the properties of a device such as retention of charge, leakage etc. $2,56,57$. As mentioned in the previous section in practice heterojunction the Fermi energy could get pinned because of the presence of trap states at the interface, leading to a deviation of the built-in potential from the theoretical value $^{58} 15,16,59$. The presence of these traps leads to fluctuation of the surface potential and can be characterized by their typical relaxation time, distribution and trap density etc. The presence of traps at the ferroelectric electrode interface is also very detrimental to the 
retention behaviour of the ferroelectric polarization especially for FET devices ${ }^{60-62}$. Hence it also becomes important to characterize the trap density levels at the interface ${ }^{7}$.

Here, we use the parallel conductance method to study the interface states ${ }^{52,63-66}$. The conductance represents the loss mechanism caused by the capture and emission of carriers from the interface traps, and is used to extract the interface state density.

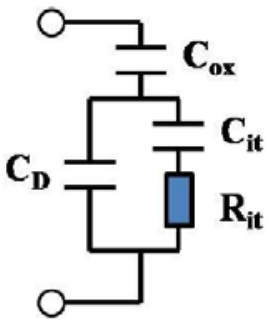

(a)

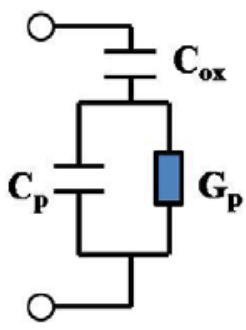

(b)

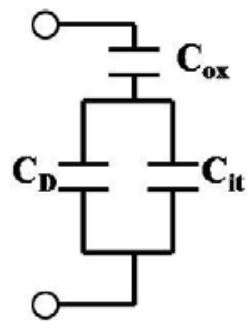

(c)

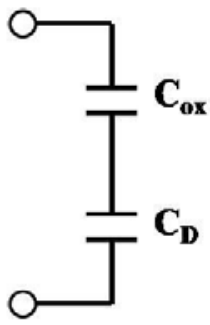

(d)

Figure 5.2. (a) (b) Equivalent circuits including interface state effects, $C_{i t}$ and $R_{i t}$. (c) Low-frequency limit. (d) High-frequency limit

In Figure $5.2(a)$, $C_{o x}$ and $C_{D}$ represent the oxide capacitance and the depletion region capacitance in the semiconductor respectively. $C_{i t}$ and $R_{i t}$ are the capacitance and resistance due to the interface states. $C_{i t} R_{i t}=\tau_{i t}$ determines the interface trap life time $\tau_{i t}$. Resistance $R_{i t}$ represents the loss due to the capture and emission of carriers from the interface traps. The trap life time $\tau_{i t}$ determines the frequency behaviour of the interface states. $R_{i t}$ is 0 in the low-frequency limit Figure 5.2(c), and $C_{D}$ is parallel to $C_{i t}$. The $C_{i t}-R_{i t}$ branch is ignored in the high-frequency limit since the interface trap life time $\tau_{\text {it }}$ is relatively long to respond to the high frequency signal ${ }^{52,67}$ Figure 5.2(d).

We measure the parallel conductance loss $\left(G_{p} / \omega\right)$ as a function of ac frequency. The parallel conductance loss is related to the interface traps as

$$
G_{p} / \omega=\left[C_{i t} / 2 \omega \tau_{p}\right] \ln \left[1+\left(\omega \tau_{p}\right)^{2}\right]
$$

Where, $C_{i t}$ is the interface trap capacitance, $\tau_{p}$ is the trap response time and $\omega$ is the applied frequency. The parallel conductance can be measured from the admittance $Y_{m}=G_{m}+j \omega C_{m}$, where $G_{m}, C_{m}$ are the measured conductance and capacitance. The measured parallel conductance $G_{m}$ is not equal to the equivalent parallel conductance $G_{p}$ due to the finite 
contribution of the ferroelectric oxide capacitance $C_{\text {ox. }}$. After subtracting the reactance due the ferroelectric capacitance the $G_{p} / \omega$ in depletion can be represented as

$$
G_{p} / \omega=\left(\omega C_{o x} G_{m}\right) /\left[G_{m}^{2}+\omega^{2}\left(C_{o x}-C_{m}\right)^{2}\right]
$$

In the above equations no fluctuation in surface potential due to non-uniformities was considered. If we consider the surface potential fluctuations from the above equations the interface density can be derived as

$$
D_{i t}=\left.\left(G_{p} / \omega\right)\right|_{\text {fmax }}\left[q A \sigma_{b}\right]^{-1}
$$

Where, $D_{i t}$ is the interface trap density, $A$ is the capacitor area, $\sigma_{b}$ is the standard deviation of the surface potential. It is assumed there that the distribution (interface state energy distribution) is Gaussian in nature and $\sigma_{b}$ is determined as described by Nicollian et $a^{65}$. In this chapter we determine the value of $D_{i t}$ as a function of gate to bulk voltage.

\subsection{Admittance angle}

Analyzing the admittance angle gives us information about the relative contribution of the capacitive and resistive elements in a MFM/MFS structure ${ }^{68}$. Moreover the frequency dependent admittance angle measurement can give information about the defect dynamics and the contribution of defects to the capacitive and resistive components. In case of dominance of defects there will be a dispersion in the admittance angle with frequency due to finite relaxation time of the defects ${ }^{7,68}$.

\subsection{Experimental Section}

For the leakage, capacitance-voltage and admittance measurements the Keithley 4200 SCS set up was used. The temperature was controlled using a Linkam LNP95 controller. For the I$\checkmark$ measurements the applied voltage was swept from 0 to $\pm 15 \mathrm{~V}$ in a stepwise manner with a step size of $0.4 \mathrm{~V}$. In order to measure the current in a steady state a dwell time of 10 secs ${ }^{13}$ was used for each voltage step. Before a measuring the I-V curve in any the sample was pre-poled for 100 secs at $+30 \mathrm{~V}$ or $-30 \mathrm{~V}$ to minimize the contribution of polarization reversal to the total measured current. For temperature dependent measurements to ensure that the sample is in thermal equilibrium the temperature was allowed to stabilize for 15 mins at 
a given temperature before commencing the measurements. The static/low frequency dielectric constant used for determining the relevant parameters was derived from the high voltage part of the P-E loops and the optical dielectric constants were taken from the literature 69,70 .

\subsection{Results and Discussion}

\subsubsection{Temperature Dependent I-V measurements}

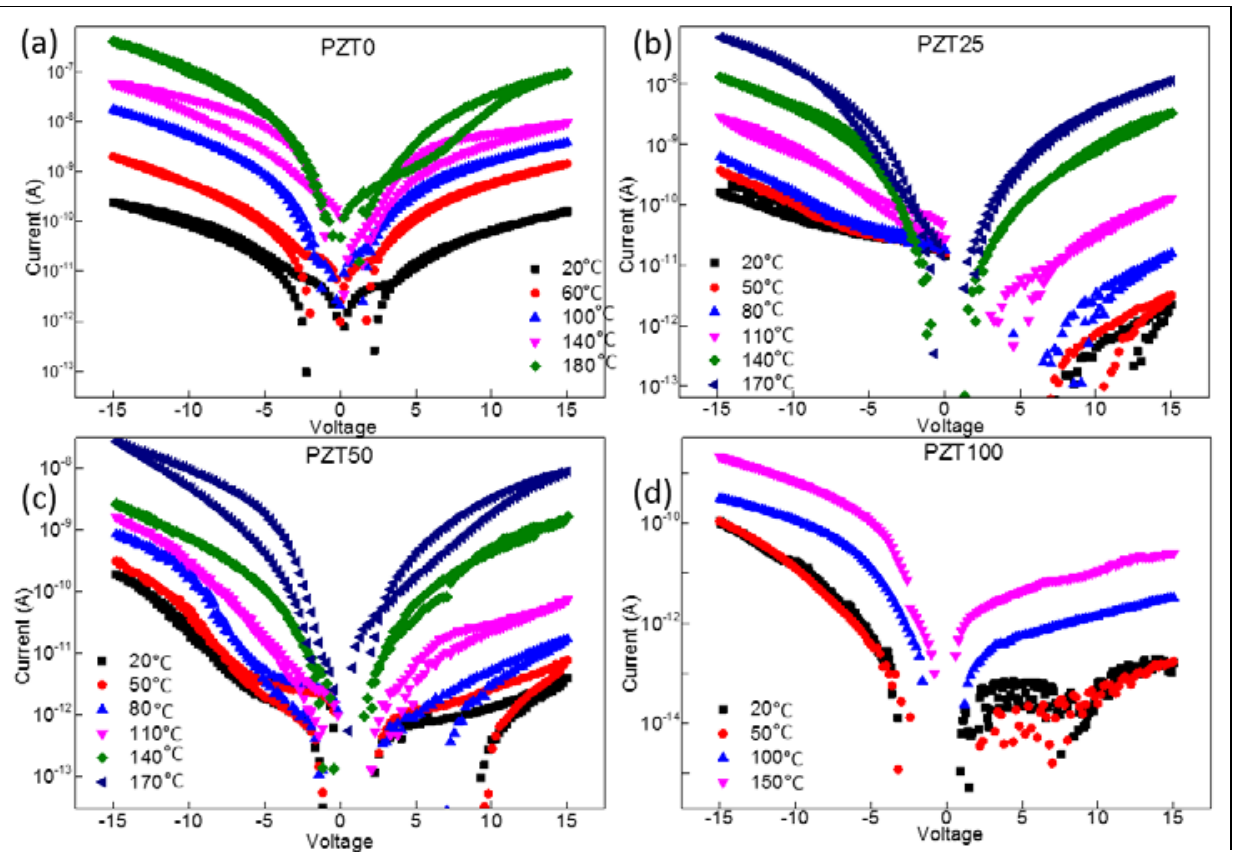

Figure 5.3 Temperature dependent leakage current as a function of applied voltage for all samples (PZT0, PZT25, PZT50, PZT100)

Above we show the temperature dependent current-voltage characteristics for all samples. We can see that for positive polarities the values of the current is much lower for PZT25, PZT50 and PZT100 samples as compared to the negative polarity which gives rise to an asymmetric I-V characteristic. Further the current for both polarities is temperature 
dependent. It can be seen that the current increases with increasing temperature. Due to the asymmetric I-V characteristic we can rule out the contribution of Poole-Frenkel emission as well as hopping conduction as the main current controlling phenomena. In addition, from the temperature dependent I-V characteristics we can rule out the role of FN tunnelling which is a temperature independent phenomena. In order to make sure that the current is not space charge (SCLC) controlled we compared the current density $(\mathrm{J})$ of the PZTO sample with that of a sample with $500 \mathrm{~nm}$ PZT. In case the SCLC is the main governing mechanism the current density would be very sensitive to the thickness of the sample Equation 2. We didn't observe any PZT thickness dependent change in the current density which ruled out SCLC mechanism as the major charge transport mechanism in these films.

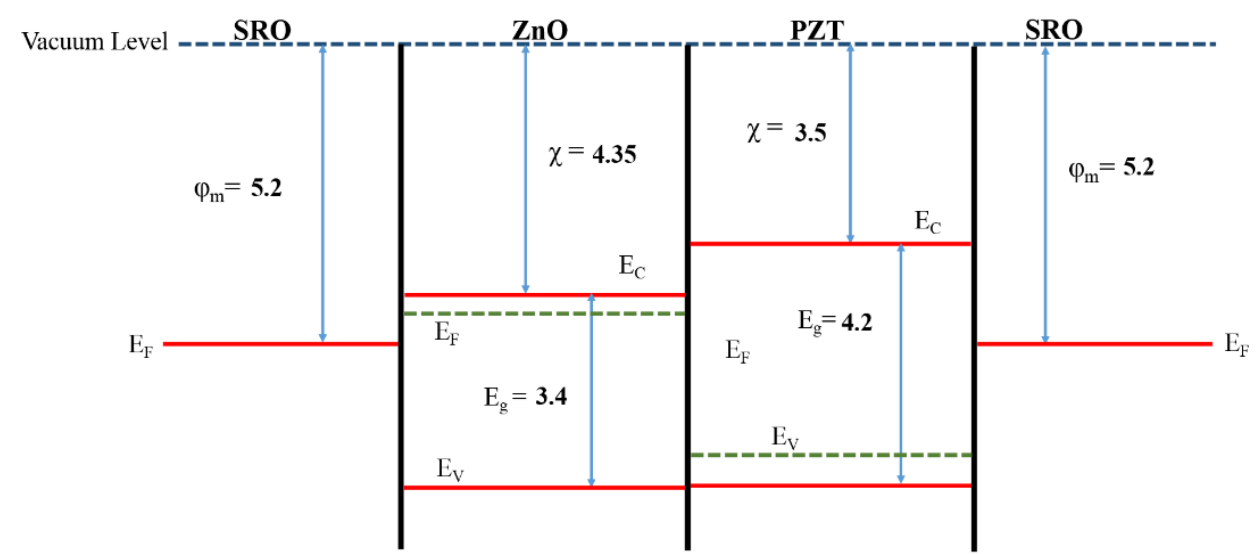

Figure. 5.4 Definition of the band alignment for the heterostructures. The mentioned energies are in $\mathrm{eV}$

From the above analysis we can rule out the role of the bulk transport mechanisms as the current controlling factor and therefore the current must be interface controlled. Hence, we can conclude that for the concerned samples interface controlled phenomena due to a different built-in potential at the two interfaces which determine the leakage current. To understand the interface built-in potentials we show in Figure 5.4 a schematic band alignment diagram. In our case we assume that the PZT is p-type and $\mathrm{ZnO}$ is $\mathrm{n}$ type doped. We shall see later why our conjectures are correct. As we can see from the above Figure 5.4 that there exists three different heterojunctions ${ }^{71}$ (1) SRO-ZnO, (2) ZnO-PZT and (3) PZT-SRO. 
Since the band bending for the $\mathrm{ZnO}-\mathrm{SRO}$ is much lower than the other two heterojunctions we can infer that the ZnO-SRO heterojunction is not the current controlling junction. Additionally, I-V measurements on $\mathrm{SRO} / \mathrm{ZnO} / \mathrm{SRO}$ structures showed that the resistance was in the range 2-10 $\Omega$ depending on the thickness of $\mathrm{ZnO}$ which is much lower than what is reported in Figure 5.3.

Hence, we analyze the I-V curve in terms of the SRO-PZT and PZT-ZnO contacts. Whereas the SRO-PZT contact can be treated as a metal-semiconductor Schottky diode the latter is a semiconductor heterojunction diode ${ }^{71,72}$. For our case for positive polarity the PZT-SRO interface (bottom) is reverse biased hence current controlling whereas for negative polarity the PZT-ZnO is reverse biased and thus current controlling. As mentioned previously PZT is very insulating therefore we use the modified Schottky-Simmons equation instead of the traditional Schottky model for the PZT-ZnO interface. We determine the height of the potential barrier via a two-step method from the temperature dependent I-V measurements as follows. Equation 9 can be simplified as $^{55}$

$$
\ln \left(J_{s s} / E T^{3 / 2}\right) \sim\left[\left(\ln \left(C^{*}\right)-q \varphi^{O_{B}} / k T\right)\right]-f\left(V^{1 / 2}\right)
$$

Where $C^{*}$ is a system dependent constant as can be easily seen from Equation 9 (depends on the mobility as well as the effective mass of the free charge carriers). The first right hand term (square brackets) is voltage independent and inversely proportional to the temperature

$F(T) \sim \ln \left(C^{*}\right)-q \varphi^{O_{B}} / k T$

In case of Schottky-Simmons controlled current the $\ln \left(\mathrm{J}_{\mathrm{s}} / E \mathrm{ET}^{3 / 2}\right)-\mathrm{V}^{1 / 2}$ should be a straight line. From the intercept of the above curves with $V=0$ axis at different temperatures the value of $\phi^{0}{ }_{B}$ can be determined using Equation 16. Below we show $\ln \left(\mathrm{J}_{\mathrm{SS}} / \mathrm{ET}^{3 / 2}\right)-\mathrm{V}^{1 / 2}$ and $\mathrm{F}(\mathrm{T}) \mathrm{vs} 1 / \mathrm{T}$ curves for PZTO and PZT25. Similar plots were obtained for the other two samples PZT50 and PZT100. 

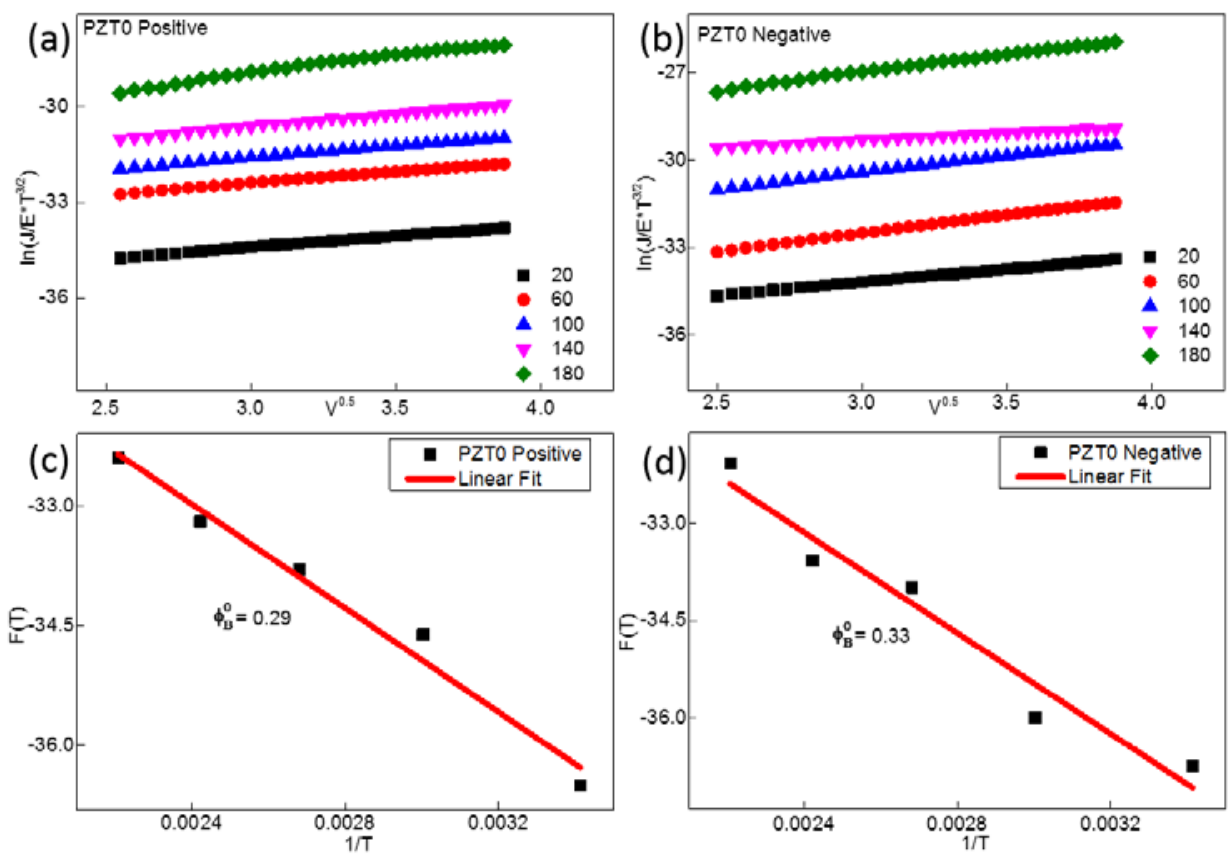

Figure 5.5. Schottky-Simmons representation of Equations. 15 and 16 for PZTO
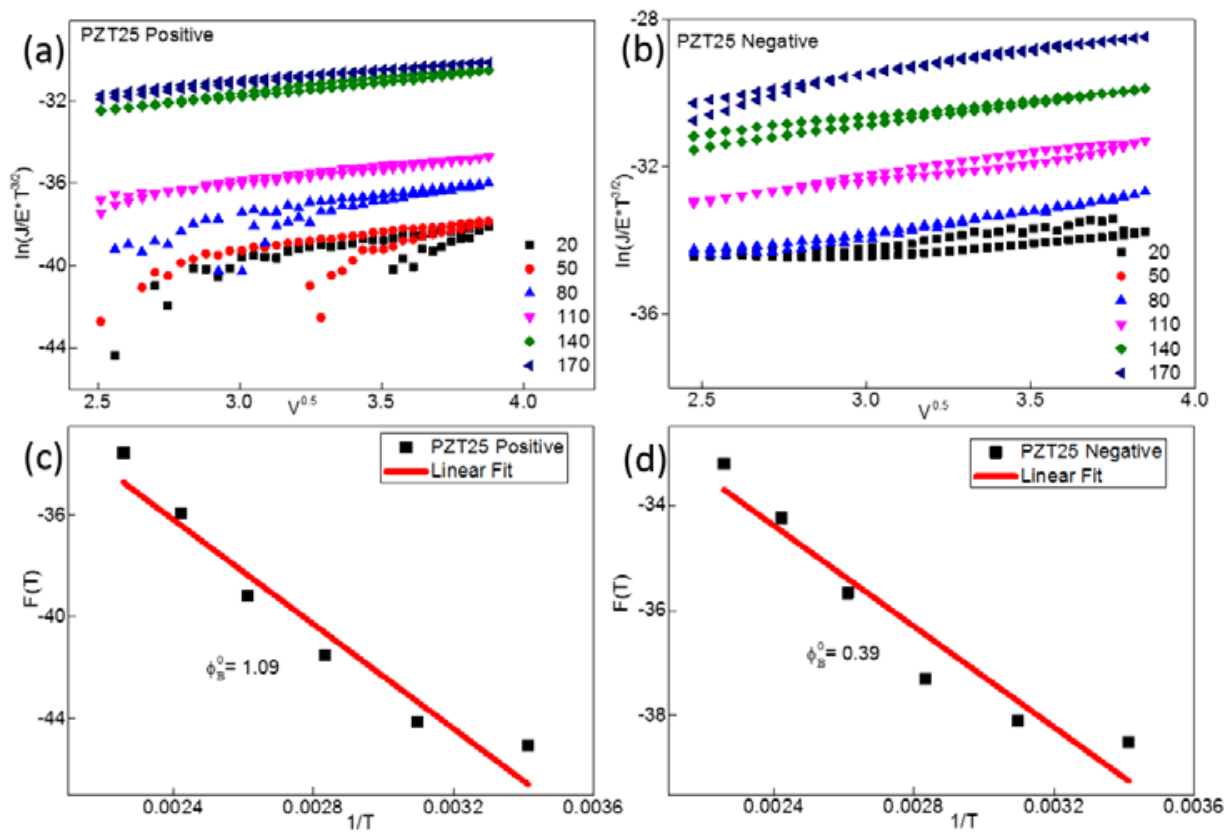

Figure. 5.6 Schottky-Simmons representation of Equations 15 and 16 for PZT25 
From the linear characteristics of the above plots we conclude that the main current controlling step is governed by the Schottky-Simmons mechanism. In Table 1 we present the height of the potential barriers as determined from the temperature dependent I-V measurements. As can be seen the reported errors for potential barrier are within $10 \%$, and have been obtained from the linear fits of $F(T)$ vs $1 / T$ curves. We can see that for negative polarity when the PZT-SRO diode is reverse biased and the current controlling step the heights of the potential barrier are almost similar in magnitude and are also in agreement with previously reported barrier height in similar systems. However for the positive polarity when the PZT-ZnO heterojunction is reverse biased and the main rate controlling we can see that the barrier heights are much higher which is also reflected in the much lower leakage currents for positive polarity.

We had previously assumed that the PZT is p-type, from analyzing the potential barrier heights we can see that our assumption is correct. In our case we can see that upon depositing the $\mathrm{ZnO}$ on top of PZT the barrier height in the positive direction on gets affected. In case the PZT was n-type doped the reverse would have been true. For n-type PZT the bottom SRO-PZT diode would be reverse biased for positive polarity unlike what is seen in our case.

\begin{tabular}{|l|l|l|l|l|}
\hline Sample & PZT0 & PZT25 & PZT50 & PZT100 \\
\hline & & & & \\
\hline $\begin{array}{l}\text { Positive polarity } \\
(\mathrm{eV})\end{array}$ & $0.29 \pm 0.03$ & $1.09 \pm 0.12$ & $1.14 \pm 0.09$ & $1.05 \pm 0.11$ \\
\hline $\begin{array}{l}\text { Negative } \\
\text { Polarity }(\mathrm{eV})\end{array}$ & $0.33 \pm 0.03$ & $0.39 \pm$ & $0.33 \pm 0.04$ & $0.36 \pm 0.05$ \\
\hline
\end{tabular}

Table 1. Effective Potential barrier heights $\phi_{\mathrm{B}}$ for both polarities for all the samples as derived from the temperature dependent current- voltage measurement. The measured error limit as determined from the standard deviation of the linear fit is also mentioned.

At low voltages we can see deviations from the linear characteristics of $\ln \left(\mathrm{J}_{\mathrm{ss}} / \mathrm{ET}^{3 / 2}\right)-\mathrm{V}^{1 / 2}$ curve. As can also be seen from Figure 5.6 (c) that at low voltage there is deviation from ideal Schottky behaviour. As can be seen from Equation 9 when the voltage is very low the 83 
exponential term is very low and the linear part which varies directly with the applied electric field dominates hence the deviation.

The above plots were also analyzed using standard Schottky conduction mechanism as in Equation 6 in similar manner as described above. The extracted potential heights were within $5 \%$ of the above reported potential heights. However, as is very commonly reported for these type of materials the effective Richardson coefficient was much lower $\sim 10^{-7} \mathrm{~A} / \mathrm{cm}^{2} \mathrm{~K}^{2}$ which is comparable to other literature ${ }^{1,45}$ reports. This significantly low value of the obtained Richardson coefficient can be ascribed to the extremely low mobility of the free charge carrier inside PZT.

The effective charge density $\mathrm{N}_{\text {eff }}$ inside the space region can be determined from the slope of the $\ln (J)-\left(V+V_{b i}\right)^{1 / 4} \operatorname{plot}^{12,13}$. It was seen that the effective charge density inside the space charge region $N_{\text {eff }}$ for PZTO for both the polarities was determined to be $\sim 5^{*} 10^{19} \mathrm{~cm}^{-3}$.

$$
\ln (J)=\left(q^{3} N_{e f f} / 8 \pi^{2} \varepsilon_{0}^{3} \varepsilon_{r} \varepsilon_{o p}^{2}\right)\left(V+V_{b i}\right)^{1 / 4}
$$

The obtained value of the $\mathrm{N}_{\text {eff }}$ is similar (lesser by a factor $\sim 2$ ) magnitude to that of the free charge concentration $\mathrm{N}_{\text {dop }}$ as obtained from the $\mathrm{C}-\mathrm{V}$ measurements (see next section). The comparable values of the $N_{\text {dop }}$ and $N_{\text {eff }}$ shows that the concentration of deep traps are not significant inside the PZT films. In case of significant presence of deep traps inside the PZT films the $\mathrm{N}_{\text {dop }}$ would be much less than $\mathrm{N}_{\text {eff. }}$. For the positive polarity for PZT25, PZT50 and PZT100 the Neff value was determined to be $\sim 2 * 10^{19} \mathrm{~cm}^{-3}$.

At very low voltages there is a small voltage region where $d l / d V$ is negative. Negative resistivity is a typical characteristics of trap emptying and filling ${ }^{1}$. This negative resistive region was predominantly observed for the positive polarity for PZT 25, PZT50 and PZT100 samples at lower temperatures. For positive polarity a depletion region at the PZT-ZnO interface region forms and free carriers are released from the traps upon the formation of the depletion layer at low voltages. At higher temperatures since the negative resistive regime decreases as the deep trap levels become activated and the charges become free to move. As we concluded earlier since the deep trap concentration in PZT is not significant the 
deep traps must be inside $\mathrm{ZnO}$. (We show later in section 5.6.3 that the interface trap concentration is also very low)

All the samples showed a hysteresis in the I-V plots for all the samples. Generally, it is seen that the current is higher for sweep down as compared to the sweep up. A gradual increase of the voltage for positive polarity leads to higher charge injection into the depletion layer. Since the measurements are also done at a relatively high dwell time the application of voltage can inject charges into the system, giving rise to higher current density during sweep down. Further, it was seen that the amount of hysteresis decreases with increase in dwell time which indicates the presence of deep traps which get occupied. For positive polarity the amount of hysteresis decreases with increase of temperature as the deep traps inside the $\mathrm{ZnO}$ already get activated. Also at higher temperatures we can see a decrease in I-V hysteresis for the positive polarity due to the activation of the deep trap levels inside the ZnO 1,55 layer.

It is however important to note here that the magnitude of leakage currents is at least an order of magnitude lower than the measured capacitive currents measured due to polarization switching for the highest measured voltage at room temperature. This observation is also reflected in our admittance measurement as reported in Section 5.6.2

\subsubsection{Temperature dependent C-V measurements}

Below in Figure 5.7 we show the $C-V$ measurements for all samples for three different temperatures, measured at $10 \mathrm{kHz}$. In our case the dc voltage was swept from $-30 \mathrm{~V}$ to $30 \mathrm{~V}$ and the value of the small signal ac field was kept at $0.1 \mathrm{~V}$. The delay time was set at $10 \mathrm{sec}$ and the step size for the dc voltage was $0.25 \mathrm{~V}$. Before the measurements the films were kept for a dwell time of 120 secs at -30 V. Ferroelectrics are normally characterized by anticlockwise butterfly loops, with two maximas which theoretically should correspond to the two coercive fields ${ }^{51}$. These capacitance peaks are associated to the polarization reversal mechanism. We can however see that the coercive field measured from the $\mathrm{C}-\mathrm{V}$ 
measurements are much lower than the ones measured using the dynamic hysteresis measurements due to the quasi-static nature of the measurements 24,25 .
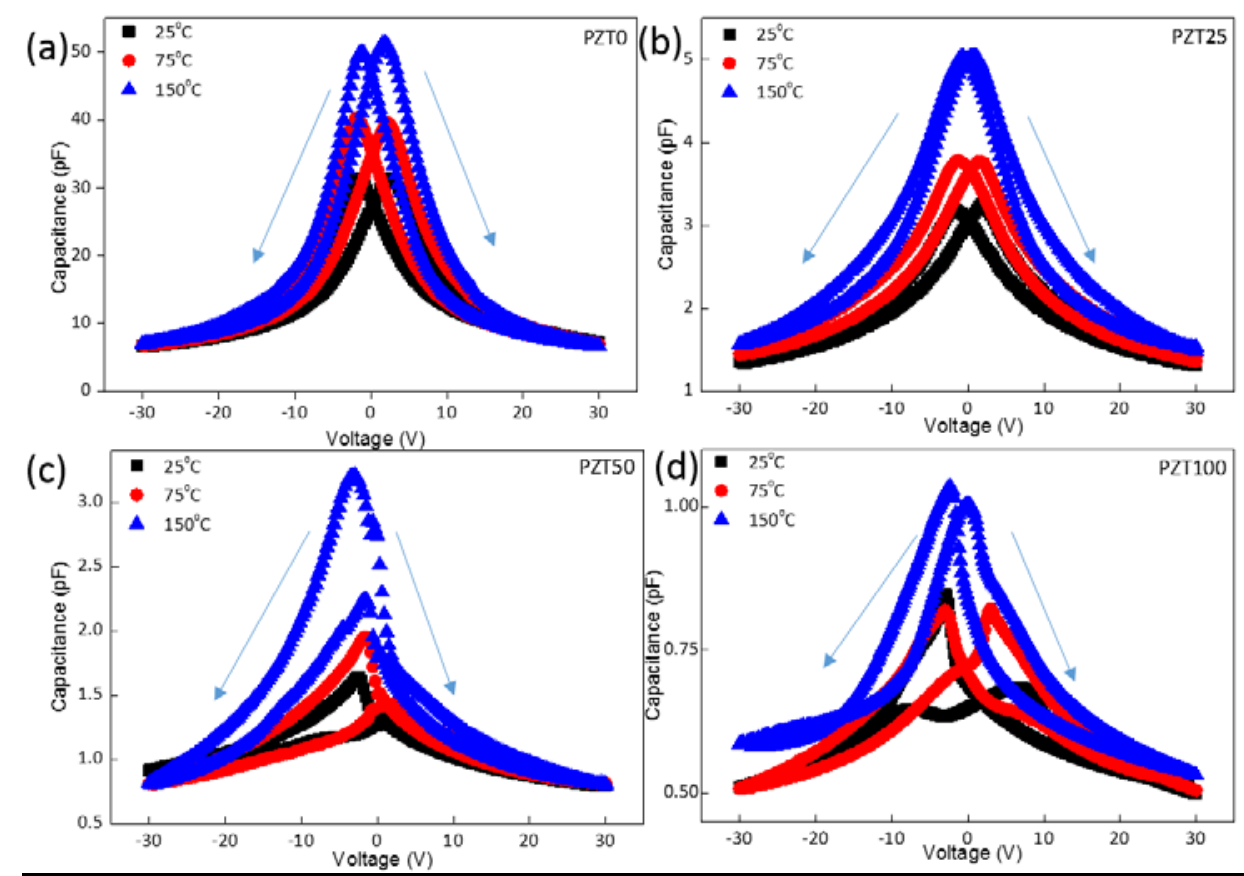

Figure. 5.7 Temperature dependent Capacitance-Voltage measurements for all the samples

We can see from the above C-V curves that the curves are anticlockwise which shows the ferroelectric capacitive characteristics of the samples. Only for PZT100 at $150^{\circ} \mathrm{C}$ above $-20 \mathrm{~V}$ we can observe clockwise characteristics, indicative of charge injection ${ }^{73,74}$. Also, we can see that the capacitance value at high voltages are almost equal in value for both polarities which shows the absence of depletion layer formation inside the $\mathrm{ZnO}$ layer $^{75}$. Additionally, as can be expected we can see that the capacitance decreases with increase in $\mathrm{ZnO}$ thickness. Also, we can see that the capacitance increases with increase in temperature for all the samples due to the increase in permittivity as can be expected for normal PZT samples (permittivity increases according to Curie-Weiss $(a w)^{76}$. It is important to note here that the value of the measured capacitance can depend also on the measurement conditions. As mentioned earlier due to the presence of the switchable polarization and long relaxation times in 
perovskite ferroelectrics, the system doesn't reach equilibrium instantaneously upon the application of the dc field. Hence, the delay to read time as well as the speed of the measurement (which determines the time for the device to reach equilibrium) needs to be optimized for a particular device ${ }^{53}$. It was observed that upon decreasing the delay times the value of the measured capacitance are higher even though the shape of the C-V curves doesn't change. Additionally, the C-V were measured over a frequency range of 4 orders of magnitude up to $10 \mathrm{MHz}$, but no significant dispersion was observed which signifies the contribution of defects is negligible for our samples.
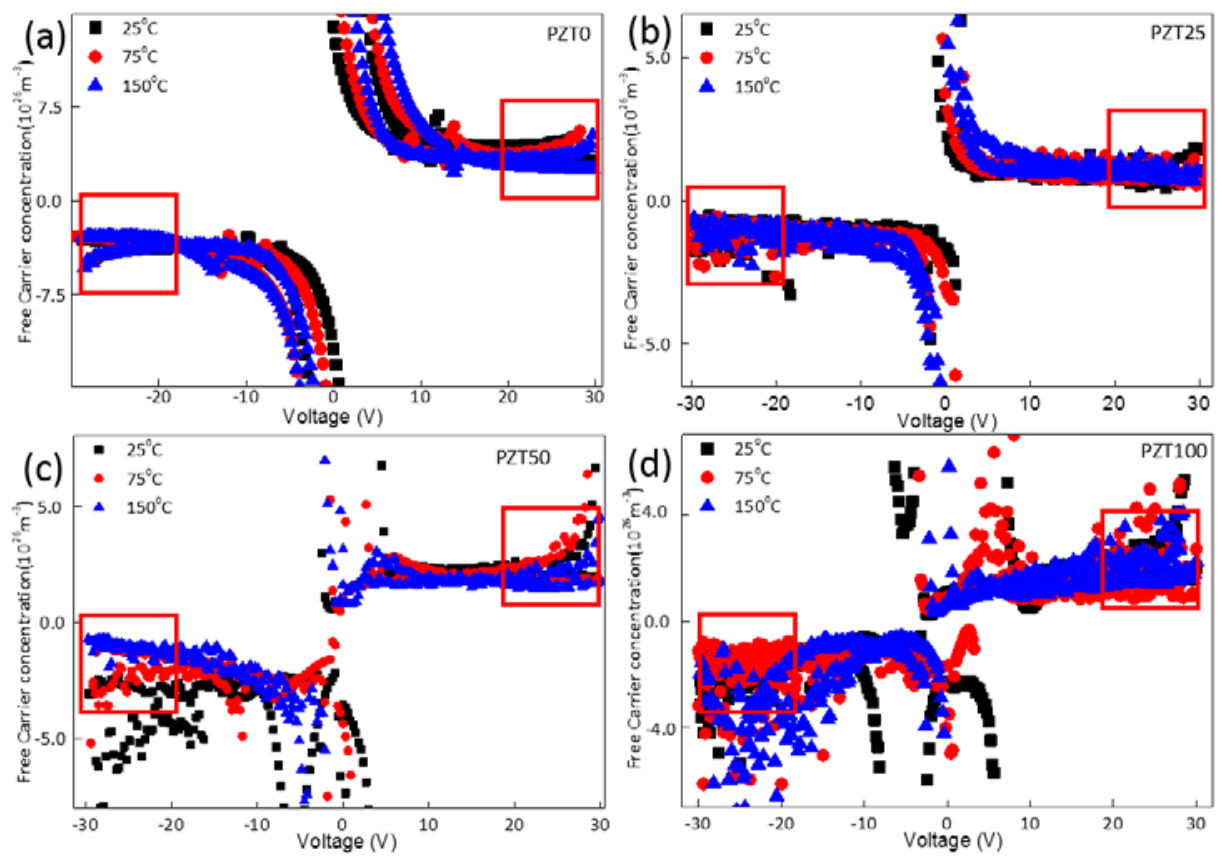

Figure. 5.8. Temperature dependent free carrier concentration from C-V measurements

In Figure 5.8 we show the free charge carrier density for different temperatures as derived from the Figure 5.7 using Equation 11. We can see that the free carrier density remains almost equal for all the samples and is in the order of $1-2 * 10^{20} \mathrm{~cm}^{-3}$ which can also be seen from the similar curvature at high voltages in Figure 5.7. Most interestingly it is also seen that the concentration of the free carriers doesn't change with temperature, which shows that 
the ferroelectric films are completely ionized even at room temperature ${ }^{13,74}$. The abrupt change of sign of the free carrier concentration near the coercive field points to the redistribution of the free charges from one electrode to the other. The negative sign for the concentration of free carriers is due to the change in sign of the derivative $d\left(1 / C^{2}\right) / d V 13,24$, 25, 53. For our purpose of estimating the free carrier concentration to eliminate the contribution from the ferroelectric switching we have only considered the high voltage regime of the $\mathrm{C}-\mathrm{V}$ measurements within the red box in Figure 5.8. It can however be seen that at high temperatures there is a slight increase in the free carrier concentration when the films have been subjected to 120 secs dwell time at - $30 \mathrm{~V}$. This points to finite field induced charge injection into the ferroelectric thin films at high temperature which is also reflected in the $\mathrm{C}-\mathrm{V}$ curves $^{73,74,77}$. As mentioned earlier about the clockwise hysteresis $\mathrm{C}-\mathrm{V}$ loop in PZT100 sample at $150^{\circ} \mathrm{C}$, we can also see from Figure. 5.8 that there is increase in the free carrier concentration inside the film.

\subsubsection{Interface trap density measurements}

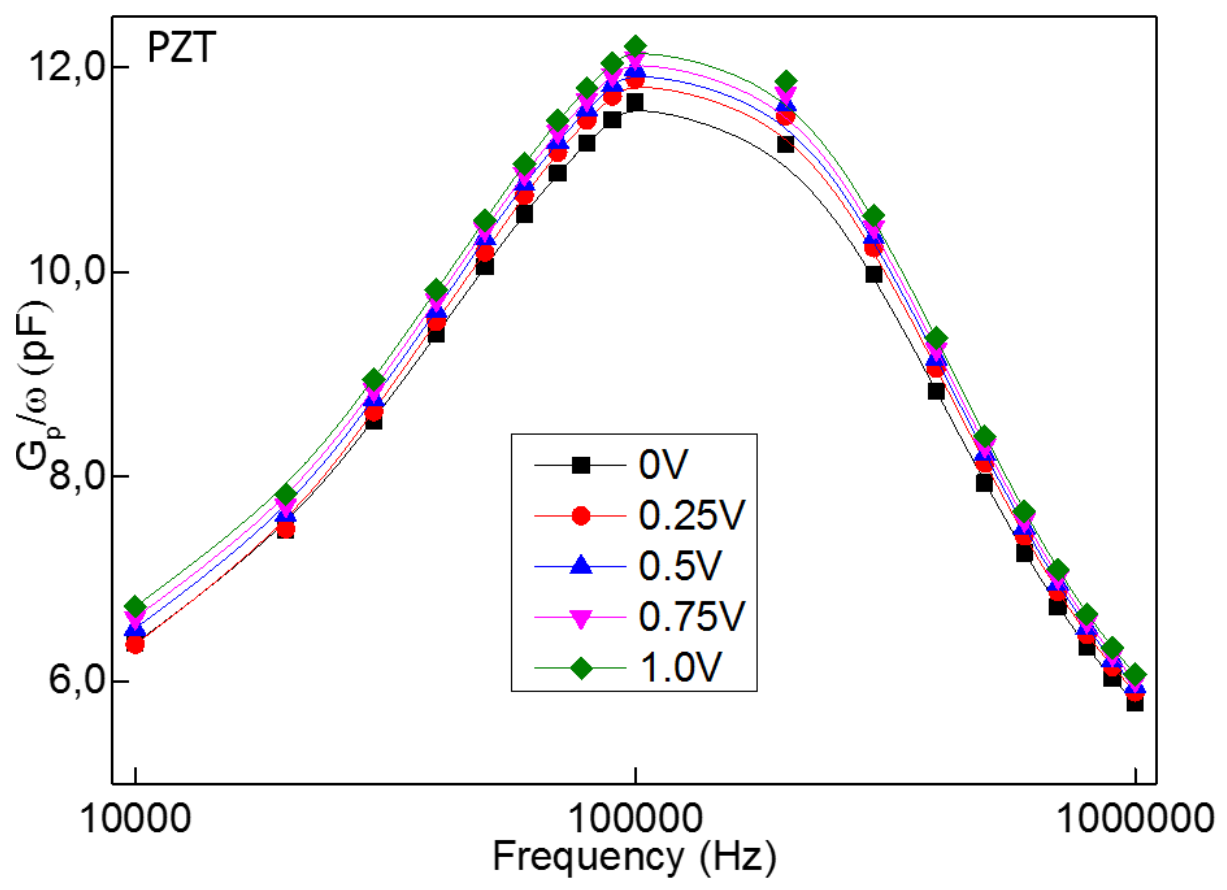

Figure. 5.9. Capacitance loss $G_{p} / \omega$ vs frequency for PZT 
As mentioned earlier measuring the parallel conductance loss can be used to derive the interface trap density. In the above Figure 5.9 we show the equivalent conductance loss $G_{p} / \omega$ as a function of frequency (see Equations 12,13). The position of the peak in the conductance loss is determined by the characteristic frequency response time $\tau_{\text {it }}$ of the traps. If the frequency of the signal is much lower than the characteristic frequency then all the traps move in phase with the ac signal causing no energy loss (as a limiting low frequency the signal can be considered as a dc voltage) as soon as the ac frequency is increased the traps lag compared to the external ac signal upto the characteristic frequency. When the frequency is increased beyond the characteristic frequency the traps are not able to respond to the ac signal anymore, leading to less energy loss (at extremely high frequencies no trap will be able to respond to the ac signal leading to zero loss). We also see that the gate to bulk voltage doesn't change the frequency characteristic of the traps, only the loss slightly increases. Similar plots were also obtained for the other samples and were used to determine the interface trap density.

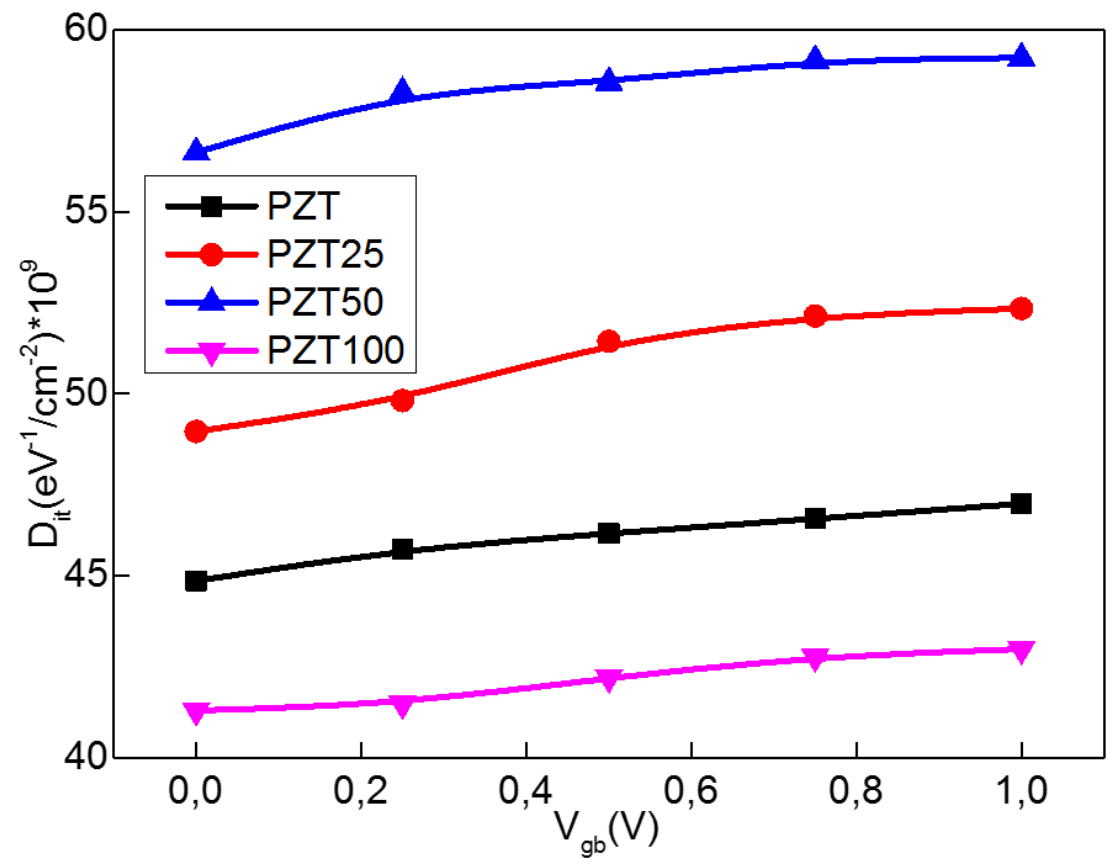

Figure. 5.10 Interface trap density vs gate voltage for all the samples for positive bias 
In Figure 5.10 we show the interface trap density derived from the curves of Figure 5.9 using Equation 14. It is seen that the interface trap density is of the order $10^{10} \mathrm{~cm}^{-2}$ for all the samples for positive bias which is couple of orders less than reported in earlier ${ }^{26}$, however the samples used in those measurements were much different than used in this thesis (Chemical solution deposition of $\mathrm{Bi}_{4} \mathrm{Ti}_{3} \mathrm{O}_{12}$ on $\mathrm{Si}$ wafers). Unlike for linear dielectrics in case of ferroelectrics we can't plot the trap density as a function of bad gap energy near the mid gap since the surface potential doesn't vary linearly with the applied gate voltage and due to the presence of switchable polarization ${ }^{78}$. The above Figure also shows that $D_{i t}$ hardly depends on the applied voltage. For the conductance measurements the measurements need to be performed in depletion, and if the leakage currents are very high they can lead to incorrect measurements especially at low frequencies.

\subsubsection{Admittance Angle}

To confirm that our measurements are dominated by capacitive contributions and not by leakage we measured admittance angle-voltage characteristics of the PZT sample from 10 kHz to $2 \mathrm{MHz}$ Figure (Figure 5.11 (a) for positive bias). 5. 11 (b) shows the admittance angles for all samples for opposite biases at $10 \mathrm{kHz}$. We can observe that the magnitude of the admittance angle is $\sim 90^{\circ}$ at all frequencies for all the devices. This points to insignificant leakage contribution in our samples as compared to the capacitive contribution. Also, the frequency independent admittance angle reflects that the overall capacitive contribution is not affected by the defect dynamics ${ }^{68}$. 


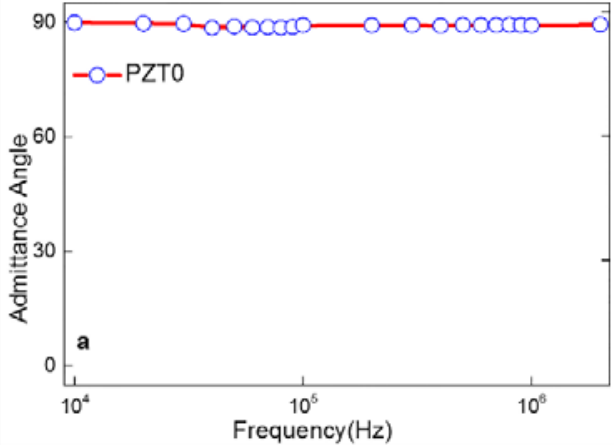

(a)

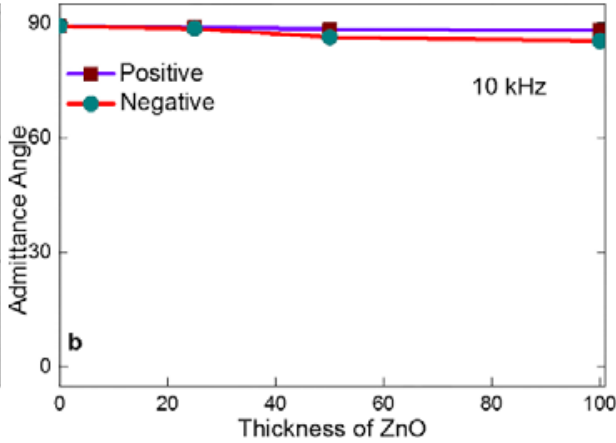

(b)

Figure. 5.11 (a) The frequency dispersion of the admittance angle between $10 \mathrm{kHz}$ and $2 \mathrm{MHz}$ of the PZTO sample and (b) Admittance angle of all the samples for opposite biases measured at $10 \mathrm{kHz}$ frequency.

\subsection{Conclusions}

In conclusion a detailed analysis of the semiconducting and transport properties MFSM heterostructures by mainly the using temperature dependent I-V and C-V measurements is presented. It was demonstrated that the capacitive contributions are much higher than the resistive leakage contribution. From the temperature dependent I-V measurements we could show that the current is governed by the PZT-SRO and PZT-ZnO interface junctions. It was also shown that the interface trap density and the deep trap levels inside the PZT is very low which otherwise could be detrimental to the device performance as an FET. It was shown that the hysteretic I-V characteristics arise because of the presence of deep traps inside the ZnO. In addition, we also determined the effective potential barriers at the two different interfaces and found them to be much lower than that imparted by the non-switchable ZnO layer as mentioned in the previous chapters. We also show that the extrinsic contribution to ferroelectric switching is negligible and the switching is intrinsic. 


\subsection{References}

1. J. F. Scott, Ferroelectric memories. (Springer Science \& Business Media, 2000).

2. C. A. Paz De Araujo, L. D. McMillan, B. M. Melnick, J. D. Cuchiaro and J. F. Scott, Ferroelectrics 104 (1), 241-256 (1990).

3. F.-C. Chiu, Advances in Materials Science and Engineering 2014, 1-18 (2014).

4. L. Pintilie, Charge transport in ferroelectric thin films. (INTECH Open Access Publisher, 2011).

5. J. F. Scott, Journal of Physics: Condensed Matter 20 (2), 021001 (2008).

6. G. Catalan, Applied Physics Letters 88 (10), 102902 (2006).

7. L. Pintilie and M. Alexe, Applied Physics Letters 87 (11), 112903 (2005).

8. M. Dawber and J. Scott, Applied Physics Letters 76 (8), 1060-1062 (2000).

9. A. Tagantsev, I. Stolichnov, E. Colla and N. Setter, Journal of Applied Physics 90 (3), 13871402 (2001).

10. J. Scott and M. Dawber, Applied Physics Letters 76 (25), 3801-3803 (2000).

11. J. Scott, F. Ross, C. P. De Araujo, M. Scott and M. Huffman, Mrs Bulletin 21 (07), 33-39 (1996).

12. L. Pintilie and M. Alexe, Journal of Applied Physics 98 (12), 124103 (2005).

13. L. Pintilie, I. Boerasu, M. J. M. Gomes, T. Zhao, R. Ramesh and M. Alexe, Journal of Applied Physics 98 (12), 124104 (2005).

14. S. M. Sze and K. K. Ng, Physics of semiconductor devices. (John wiley \& sons, 2006).

15. W. Mönch, Surface science 299, 928-944 (1994).

16. W. Mönch, Physical Review Letters 58 (12), 1260-1263 (1987).

17. M. Raymond and D. Smyth, Journal of Physics and Chemistry of Solids 57 (10), 1507-1511 (1996).

18. S. Aggarwal and R. Ramesh, Annual review of materials science 28 (1), 463-499 (1998).

19. A. R. West, Solid state chemistry and its applications. (John Wiley \& Sons, 2007).

20. V. Yarmarkin, B. Gol'tsman, M. Kazanin and V. Lemanov, Physics of the solid state 42 (3), 522-527 (2000).

21. G. W. Dietz and R. Waser, Integrated Ferroelectrics 8 (3-4), 317-332 (1995). 
22. U. Özgür, Y. I. Alivov, C. Liu, A. Teke, M. A. Reshchikov, S. Doğan, V. Avrutin, S. J. Cho and H. Morkoç, Journal of Applied Physics 98 (4), 041301 (2005).

23. H. S. Kang, Journal of Applied Physics 95 (3), 1246 (2004).

24. L. D. Filip, L. Pintilie, V. Stancu and I. Pintilie, Thin Solid Films 592, 200-206 (2015).

25. L. Pintilie, M. Lisca and M. Alexe, Applied Physics Letters 86 (19), 192902 (2005).

26. M. Alexe, Applied Physics Letters 72 (18), 2283 (1998).

27. J. Scott, Journal of Physics: Condensed Matter 26 (14), 142202 (2014).

28. A. Costa, R. Kiminam and M. Morelli, (American Scientific Publishers, 2008).

29. A. Rose, Physical Review 97 (6), 1538-1544 (1955).

30. M. A. Lampert, Physical Review 103 (6), 1648-1656 (1956).

31. P. Mark, Journal of Applied Physics 39 (4), 2163 (1968).

32. M. Suzuki, Journal of Physics and Chemistry of Solids 41 (11), 1253-1260 (1980).

33. G. Pike, Physical Review B 6 (4), 1572 (1972).

34. P. W. M. Blom, R. M. Wolf, J. F. M. Cillessen and M. P. C. M. Krijn, Physical Review Letters 73 (15), 2107-2110 (1994).

35. W. Schottky, Naturwissenschaften 26 (52), 843-843 (1938).

36. W. Schottky, Phys Z 41, 570 (1940).

37. E. H. Rhoderick and R. H. Williams, Metal-semiconductor contacts. (Clarendon Press Oxford, 1988).

38. M. L. Cohen, Journal of Vacuum Science \& Technology 16 (5), 1135-1136 (1979).

39. A. Dimoulas, P. Tsipas, A. Sotiropoulos and E. Evangelou, Applied physics letters 89 (25), 252110-252110 (2006).

40. A. Cowley and S. Sze, Journal of Applied Physics 36 (10), 3212-3220 (1965).

41. J. Bardeen, Physical Review 71 (10), 717 (1947).

42. D. K. Schroder, Semiconductor material and device characterization. (John Wiley \& Sons, 2006).

43. J. G. Simmons, Physical Review Letters 15 (25), 967-968 (1965).

44. J. Simmons, Journal of Physics D: Applied Physics 4 (5), 613 (1971).

45. L. Pintilie, I. Vrejoiu, D. Hesse, G. LeRhun and M. Alexe, Physical Review B 75 (10) (2007). 
46. I. Pintilie, C. M. Teodorescu, C. Ghica, C. Chirila, A. G. Boni, L. Hrib, I. Pasuk, R. Negrea, N. Apostol and L. Pintilie, ACS Appl Mater Interfaces 6 (4), 2929-2939 (2014).

47. P. Maksymovych, S. Jesse, P. Yu, R. Ramesh, A. P. Baddorf and S. V. Kalinin, Science 324 (5933), 1421-1425 (2009).

48. M. Lenzlinger and E. Snow, Journal of Applied physics 40 (1), 278-283 (1969).

49. A. Chanthbouala, V. Garcia, R. O. Cherifi, K. Bouzehouane, S. Fusil, X. Moya, S. Xavier, H. Yamada, C. Deranlot, N. D. Mathur, M. Bibes, A. Barthelemy and J. Grollier, Nature materials 11 (10), 860-864 (2012).

50. Z. Hu, Q. Li, M. Li, Q. Wang, Y. Zhu, X. Liu, X. Zhao, Y. Liu and S. Dong, Applied Physics Letters 102 (10), 102901 (2013).

51. C. J. Brennan, Integrated Ferroelectrics 2 (1-4), 73-82 (1992).

52. E. H. Nicollian, J. R. Brews and E. H. Nicollian, MOS (metal oxide semiconductor) physics and technology. (Wiley New York et al., 1982).

53. L. Pintilie, L. Hrib, I. Pasuk, C. Ghica, A. Iuga and I. Pintilie, Journal of Applied Physics 116 (4), 044108 (2014).

54. L. Pintilie, C. Ghica, C. M. Teodorescu, I. Pintilie, C. Chirila, I. Pasuk, L. Trupina, L. Hrib, A. G. Boni, N. Georgiana Apostol, L. E. Abramiuc, R. Negrea, M. Stefan and D. Ghica, Scientific reports 5, $14974(2015)$.

55. L. Pintilie, V. Stancu, L. Trupina and I. Pintilie, Physical Review B 82 (8) (2010).

56. Y. Watanabe, Applied Physics Letters 66 (14), 1770 (1995).

57. R. Ramesh, W. Chan, B. Wilkens, H. Gilchrist, T. Sands, J. Tarascon, V. Keramidas, D. Fork, J. Lee and A. Safari, Applied physics letters 61 (13), 1537-1539 (1992).

58. A. Guerrero, L. F. Marchesi, P. P. Boix, S. Ruiz-Raga, T. Ripolles-Sanchis, G. GarciaBelmonte and J. Bisquert, ACS nano 6 (4), 3453-3460 (2012).

59. V. Schmidt, S. Senz and U. Gösele, Applied Physics A 86 (2), 187-191 (2007).

60. P. Wurfel and I. P. Batra, Physical Review B 8 (11), 5126-5133 (1973).

61. S. Y. Wu, Ferroelectrics 11 (1), 379-383 (1976).

62. T. Ma and J.-P. Han, Electron Device Letters, IEEE 23 (7), 386-388 (2002).

63. E. H. Nicollian and A. Goetzberger, Applied Physics Letters 7 (8), 216 (1965).

64. J. Simonne, Solid-State Electronics 16 (1), 121-124 (1973). 
65. E. Nicollian, A. Goetzberger and A. Lopez, Solid-State Electronics 12 (12), 937-944 (1969). 66. W. Hill and C. Coleman, Solid-State Electronics 23 (9), 987-993 (1980).

67. W. C. Kao, (2010).

68. A. I. Khan, K. Chatterjee, B. Wang, S. Drapcho, L. You, C. Serrao, S. R. Bakaul, R. Ramesh and S. Salahuddin, Nature materials 14 (2), 182-186 (2015).

69. B. Nagaraj, S. Aggarwal and R. Ramesh, Journal of Applied Physics 90 (1), 375 (2001).

70. M. P. Moret, M. A. C. Devillers, K. Wörhoff and P. K. Larsen, Journal of Applied Physics 92 (1), 468 (2002).

71. H. Kroemer, ChemPhysChem 2 (8-9), 490-499 (2001).

72. W. Frensley and H. Kroemer, Physical Review B 16 (6), 2642 (1977).

73. N. Maffei and S. Krupanidhi, Journal of applied physics 72 (8), 3617-3621 (1992).

74. L. Pintilie, C. Dragoi, R. Radu, A. Costinoaia, V. Stancu and I. Pintilie, Applied Physics Letters 96 (1), 012903 (2010).

75. W. Choi, S. Kim, Y. W. Jin, S. Y. Lee and T. D. Sands, Applied Physics Letters 98 (10), 102901 (2011).

76. M. Dawber, K. M. Rabe, and J. F. Scott., Reviews of modern physics 77 (4) (2005).

77. E. Cagin, D. Y. Chen, J. J. Siddiqui and J. D. Phillips, Journal of Physics D: Applied Physics 40 (8), 2430-2434 (2007).

78. H. Z. Massoud, Microelectronic engineering 36 (1), 95-98 (1997). 


\section{CHAPTER 6}

\section{MESO-FREQUENCYSTUDY OF SWITCHING \\ DYNAMICS IN PZT-ZNO HETEROSTRUCTURES}

The dynamic coercive fields of PZT and PZT-ZnO heterostructures used in previous chapters were measured as a function of frequency $\left(10-10^{4}\right) \mathrm{Hz}$ and temperature (77$298 \mathrm{~K})$. We observe for the measured temperature range two scaling regimes in the $\log \left(\mathrm{E}_{\mathrm{c}}\right)$ versus $\log$ (frequency) plot for the PZT0 sample. The two scaling regimes arise from the dynamic cross over from the thermally activated to the flow regime of the ferroelectric domain wall motion. We also constructed a dynamic temperature (T) - electric field (E) phase diagram of the ferroelectric switching dynamics for the PZT0 sample. For the PZT 25, PZT50 and PZT100 samples the two scaling regimes are not prominently visible for the positive coercive field direction, whereas in the negative direction two different scalings similar to that of PZT0 is observed. Also, the coercive field in the positive direction is less sensitive to the temperature unlike the coercive field in the negative direction. 


\subsection{Introduction}

As previously mentioned in Chapter 4, ferroelectric domain reversal involving domain wall motion can be considered as the movement of an elastic interface in a disordered medium under an applied electric field ${ }^{1-6}$. Understanding the static and dynamic behaviour of domain walls, and specifically their interaction with random (defects/disorder) pinning sites is important to understand, and to control, domain switching, growth and stability, all questions of high technological as well as fundamental interest. This random pinning potential landscape is caused by dipole defects (random bond and random field disorders) 7 , 8 , which act as pinning centres for domain wall movement ${ }^{9}$. This competition between the elasticity, which tends to flatten the interface, and pinning, that induces a fluctuating potential energy landscape, gives rise to a complex dynamical response to the applied electric field ${ }^{2}, 10$. Even though first principle calculations have been able to provide accurate estimates of domain wall energies and defect interaction energies, it has not been possible to scale up the simulation sample size to reproduce realistic conditions ${ }^{11,12}$. Hence a statistical treatment of the random potential fluctuations at multiple length scales is necessary. This kind of simplified treatment of domain walls can be a very useful technique to study such a disordered elastic system and addresses the glassy nature arising from the multiple local energy minima4, 5 .

The ferroelectric domain reversal mechanism involving nucleation and growth generally takes place in the meso-frequency $\left(10^{1}-10^{7} \mathrm{~Hz}\right)$ region $9,13,14$. As was shown in Chapter 4 , the domain reversal can be either nucleation or growth/propagation controlled, and in the mesotemporal region the competition between these mechanisms can give rise to very interesting switching dynamics. Hence, dynamic, meso-frequency domain wall motion studies can be very helpful in understanding the underlying domain reversal dynamics in ferroelectrics. The effect of an external force on an elastic interface in a disordered potential can be categorised into three regimes, depending on the magnitude of the applied force (field) at a finite temperature: a) the pinning regime; b) the temperature assisted creep regime and c) the flow regime ${ }^{1,4}$. These three different voltage regimes have three different domain wall velocity- electric field relationships: $v=0$ (when pinned at very low electric fields), $\log (v) \sim$ 
$E_{\text {ext }}$ (creep at intermediate electric fields), $v \sim E_{\text {ext }}$ (slide at high electric fields) as illustrated in Figure 6.1.4, 5

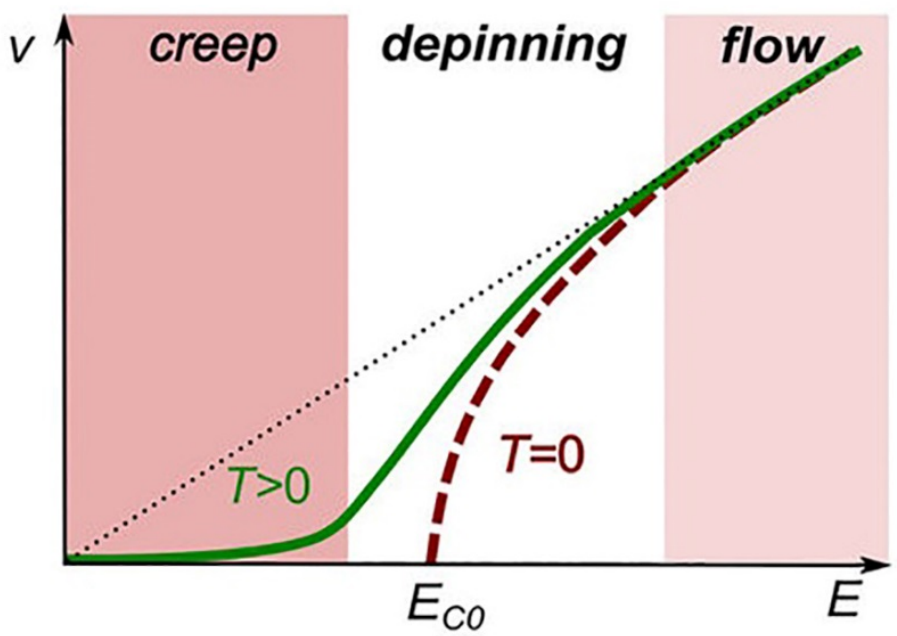

Figure 6.1. Illustration of the dynamic response of a pinned elastic interface. Interface velocity as function of the driving external electric field. ${ }^{15}$ (Reproduced with permission)

In the above curve at $0 \mathrm{~K}$ temperature the interface starts to move once the applied electric field exceeds the critical field $E_{\mathrm{co}}$, at finite temperature thermally activated slow interface motion is possible even at sub-critical fields.

In Figure 6.2 we illustrate the energy landscape in a disordered medium based on Nattermann et al's ${ }^{10}$ work and the various mechanisms of domain wall motion for different applied fields. 

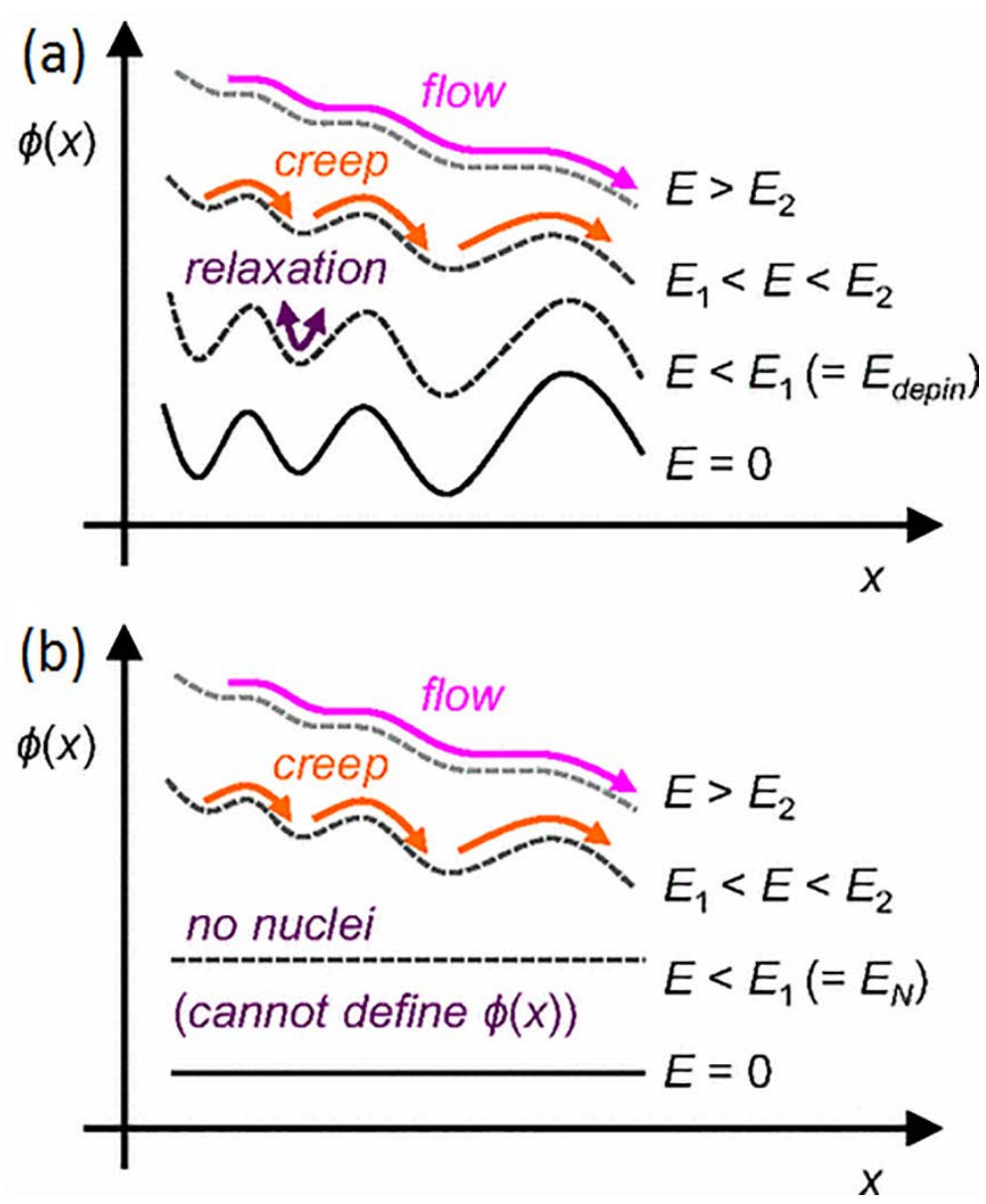

Figure 6.2. Energy landscapes $\phi(x)$ for various domain wall motion regimes as function of the applied electric field $E$ in a random medium. (a) For the case when domain wall exist initially and (b) when they do not exist initially. ${ }^{16}$ (Reproduced with permission)

In Figure 6.2(a) it is shown that a system, in which initially domain walls already exist, is a disordered system, in which the energy landscape varies from one point to the next, with minima caused by randomly positioned pinning centres. For very small fields the domain walls can only move inside a local potential well without any finite drift. With a gradual increase of the applied electric field at finite temperatures the domain walls can hop into a next potential well, giving rise to the creep regime. At much higher fields the domain wall motion goes over into the flow regime. In case no pre-existing domain walls are present, a potential landscape $\phi(x)$ cannot be defined. Initial reverse domains need to be nucleated for 
which the nucleation barrier has to be overcome ${ }^{15}, 16$. Hence, as shown in Figure $6.2(\mathrm{~b})$, the thermally activated creep motion can occurafter crossing the nucleation barrier $E_{N}$. For large fields the system enters again the flow regime.

In Chapter 4 we showed that the local activation energy is very sensitive to the local dipole strength (the switching activation field increases exponentially with a linear increase in builtin field), hence it would be very interesting to see if the system can go directly from the pinned regime into the flow regime, by making the nucleation barrier much higher (compared to $\mathrm{kT}$ at room temperature) and in this way suppress the thermally activated creep regime and the domain wall depinning.

Mathematically pulse based measurements (as discussed in Chapter 4) and frequency dependent studies are equivalent, since one is the Fourier transform of the other ${ }^{13}$. However in practice they are quite different. In pulse based measurements, as performed in Chapter 4, a constant magnitude field is applied to the film, whereas in the case of frequency dependent hysteresis measurements the film is subjected to a changing field which does not allow the system to reach equilibrium (equilibrium here means reaching steady state velocity for a given applied field). Normally, in pulse based measurements there is a delay time between the write and read voltage pulse, which allows enough time for the moving domain walls to come to a halt due to the internal viscosity (for details see Chapter 4). In frequency dependent hysteresis measurements however, there is no continuous increase of electric field from 0 to maximum applied voltage $V_{\max }$, rather the voltage sweep for a complete loop is made by a fixed number of voltage steps (in this thesis the number of steps is 400 ), hence for a $1 \mathrm{kHz}$ measurement the sample stays at any particular voltage for $2.5 \mu \mathrm{s}^{17}$.

For technological and application reasons frequency dependent hysteresis behaviour of ferroic systems have been studied in great detail especially in ferromagnetic systems. Over a century ago Rayleigh 18,19 and Steimetz 18,20 proposed a scaling relationship between the coercive field of a hysteresis loop and the sweep rate (f) of the magnetic field: [ $E_{c} \sim f^{\alpha}$, where $\alpha$ is a scaling factor]. Rao et al. gave the first theoretical explanation of this frequency dependent scaling from a microscopic point of view by considering the system as a 2 or 3 dimensional Ising ${ }^{21}$. Here it is noteworthy to mention that the scaling coefficient $\alpha$ does not represent any physical quantity, but defines the rate at which the area of the loop grows as 
a function of applied frequency. Later it was noticed that a single scaling law was not enough to describe the observed frequency dependence because the theoretical models did not take into account the domain wall dynamics, which dominates the magnetization process ${ }^{14,22,23}$. Further the predicted theoretical values of $\alpha$ were orders of magnitude larger than the experimentally observed values $14,22,23$. Later work by Racquet et al ${ }^{24,25}$ and Bland et al ${ }^{14}$ showed that the observed phenomenon (the presence of two different scaling parameters) can be explained by considering two separately acting mechanisms, namely domain nucleation and domain wall motion. From their analysis they concluded that at low frequency/sweep rate the domain wall motion is the main domain reversal mechanism, while at higher sweep rate domain nucleation is the dominant reversal mechanism.

Previous work on the frequency dependence of the coercive field in ferroelectrics was based on the work of Ishibashi et al, and Chen et al. While Ishibashi et al. 26, 27 considered unrestricted domain growth (based on the Avrami theory as the main mechanism), Chen et al. ${ }^{28}$ proposed that the nucleation is the main rate determining step. Later experimental results by Scott et al. ${ }^{29}$ showed that their results fitted moderately better with Chen's model. But none of these above two works (Chen et al. ${ }^{28}$ and Ishibashi et al. ${ }^{27}$ ), unlike in magnetism, considered the nucleation and growth mechanism separately, nor their temperature dependence. Moreover, Chen et al. ${ }^{28}$ and Ishibashi et al. ${ }^{27}$ did not consider domain wall motion as an elastic disorder media problem. Because of the analogy between the underlying physics of domain reversal in a disordered media in ferromagnetics and that in ferroelectrics, we decided to use the analysis adopted for ferromagnetism to analyse our results in ferroelectric systems.

As discussed in Chapter 4 we need to consider the role of nucleation and growth mechanism separately, therefore we followed the analysis of Raquet et al ${ }^{24,25}$ and Bland et al ${ }^{14}$ to distinguish the role of domain nucleation and growth in domain reversal from the presence of two different scaling parameters. Further, using the temperature $(T)$ and frequency dependent P-E hysteresis loops we construct the T-E dynamic phase diagram of the PZTO sample. This diagram, as proposed by Nattermannn et al. ${ }^{10}$, and shown in Figure 6.3, can give a complete description of how the domain reversal takes place in a disordered elastic system under the application of an ac field. The solid (red) $E_{1}$ and (blue) $E_{2}$ lines demarcate the 
dynamic crossover lines, separating the relaxation and creep regimes and the creep and flow regimes, respectively. The horizontal (green) $E(t)$ line denotes the first quarter period, 1/4f, of an $E(t)$ cycle, of the P-E hysteresis loop measurement. The frequency dependence of the P-E hysteresis loop correspond to how fast the $E(t)$ line is swept. The dotted (black) line separates a critical depinning region from other dynamic regimes (not specified further here), occurring at low T.

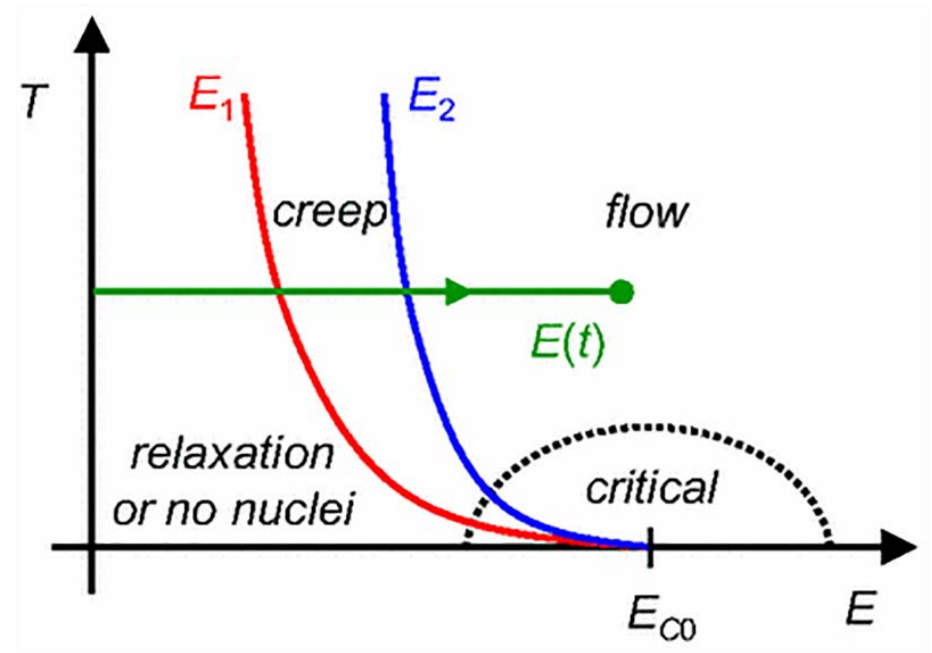

Figure 6.3. Dynamic phase diagram of domain wall dynamics as a function of the temperature and electric field. ${ }^{16}$ (Reproduced with permission)

Figure 6.3 can be related directly with Figure 6.1 where at $0 \mathrm{~K}$ temperature the walls remain completely pinned, at finite temperature and intermediate fields the domain wall starts to move in a thermally activated manner (creep) and at much higher fields the domain wall motion goes over into the flow regime.

In the above analysis by Nattermannn et al. ${ }^{10}$ the authors also mention that the creep regime is in practice a thermally activated regime and can therefore also be classified as a thermally activated nucleation regime. The reason they attributed terming this thermally activated regime to creep and not to thermally activated nucleation was that the activation takes place at the interface of the domain wall (not to be confused with the ferroelectric-electrode domain wall but the interface between two domain walls in the bulk), whereas in their case and all the previous models considered nucleation as a bulk phenomenon. Nattermannn et 
al. ${ }^{30}$ also note that if there exists a distribution of energy barriers on varying length scales, then the activation energy will vary as a power function of the applied magnetic field. These same criteria can also be applied for spatially varying heterogeneous nucleation barriers (at the ferroelectric-electrode interface), since the nucleation takes place at the ferroelectricelectrode interface and the spatial variation can be due to distribution of barriers at this interface. Noh et al. ${ }^{15,16}$ use the creep terminology to describe their observation of double scaling (in the coercive field vs frequency plots); however they did not address the above question why the thermally activated nucleation is not considered. Hence subsequently in this chapter we do not use the term creep for the intermediate thermally activated regime between $E_{1}$ and $E_{2}$, but refer to this regime as the "thermally activated regime" (TAR). Even though in the previous literature reports and also in Chapter 4 the velocity vs electric field relation is used and classified the low voltage regimes in the PZTO and WDRU for all the samples as the creep regime, it remains an open question whether it is actually creep or thermally activated heterogeneous nucleation. Because of the absence of any theoretical work taking into account the role of heterogeneous nucleation in disordered elastic media dynamics, it would be not be pertinent to categorically assign these regimes as creep.

In this chapter we also show that the scaling (log $\left(E_{c}\right)$ versus log (frequency) plot) varies with increasing nucleation activation energy and how the reversal field varies depends on the temperature (if the reversal field is thermal or athermal).

\subsection{Experimental Section}

The frequency dependent hysteresis measurements were performed using the TF analyzer 3000 (aiXACCT). The temperature was controlled using the Linkam LNP95 controller. An average over 10 hysteresis measurements was performed to obtain a better representative value of the coercive field. In order to avoid incomplete switching the maximum applied electric field was at least 2.5 times $E_{c}$. 


\subsection{Results and Discussion}

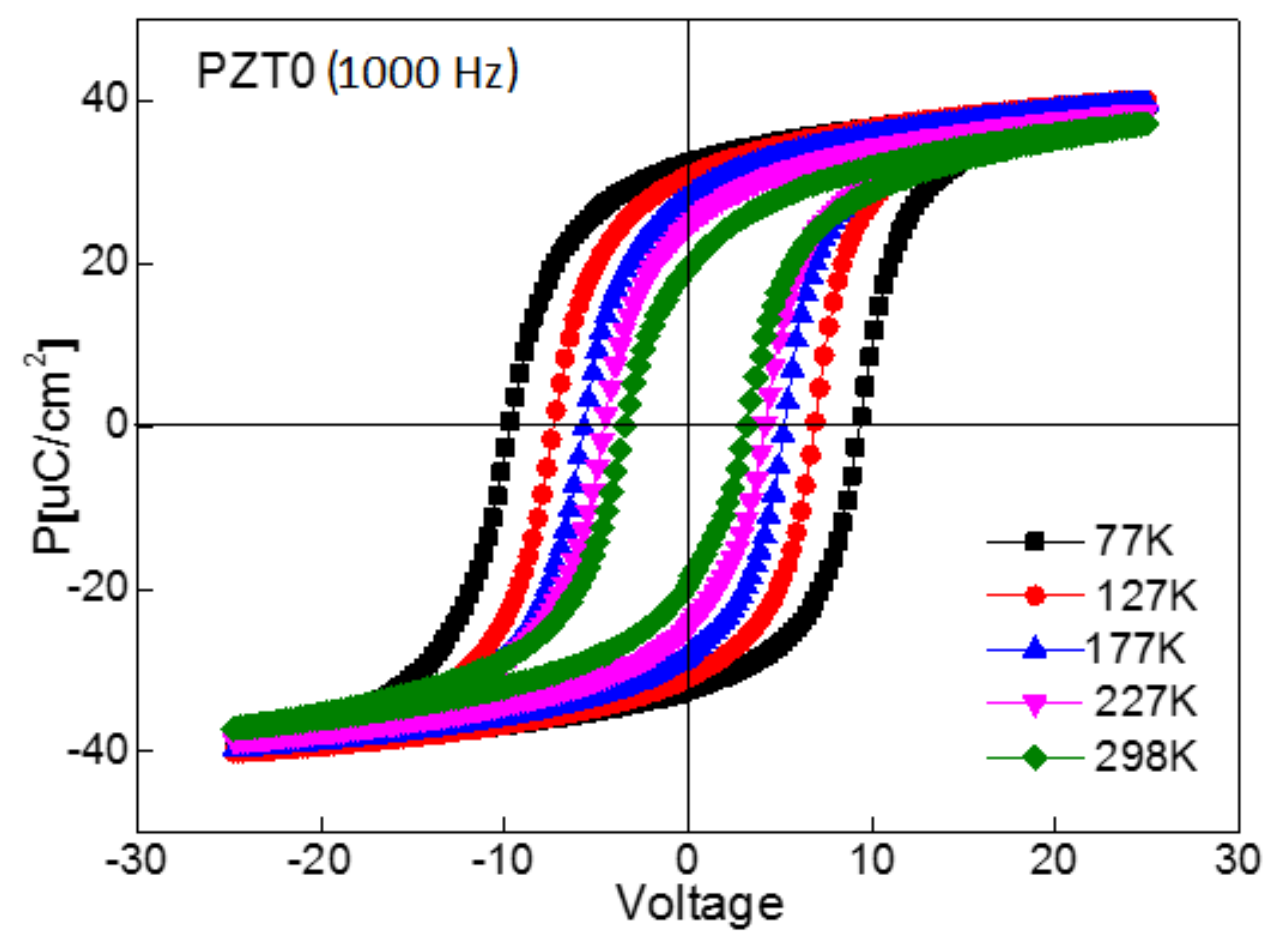

Figure 6.4. Temperature dependent dynamic hysteresis loop of the PZTO sample from 77K to 298K measured at $10^{3} \mathrm{~Hz}$.

Figure 6.4 shows the P-E hysteresis loops of the PZTO sample as a function of temperature. It is seen that the coercive voltage increases with decreasing temperature and that the shape of the loop does not change (there is second order change in shape due to domain dynamics will be discussed later). The decrease in the value of the coercive field with temperature shows that the ferroelectric switching is a thermally activated process. We also notice that, even though the saturation polarization $\mathrm{P}_{\mathrm{s}}$ value hardly changes with the temperature, the remnant polarization $\mathrm{Pr}$ increases with decreasing temperature. This also indicates that the loss in polarization, due to back-switching, is also a thermally activated process. 


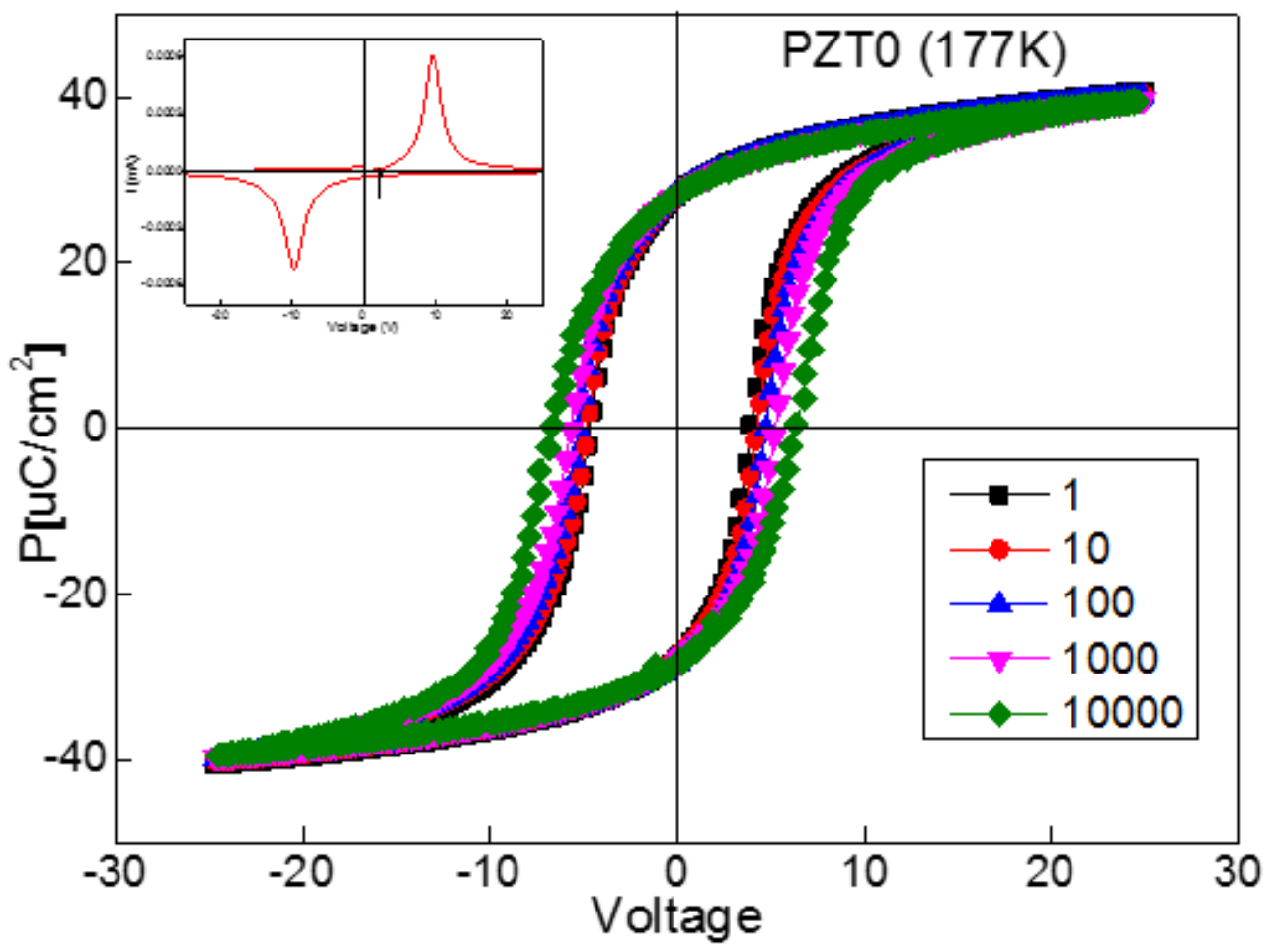

Figure 6.5. Frequency dependent dynamic hysteresis loop of the PZTO sample from $1 \mathrm{~Hz}$ to $10^{4} \mathrm{HZ}$ measured at $177 \mathrm{~K}$. The inset shows the switching current at $10^{3} \mathrm{~Hz}$. The arrow inside the inset shows the onset of nucleation.

In Figure 6.5 we show the hysteresis loops of PZTO as a function of the frequency. We can see that the slope of the loops at $E_{c}$ increases with decreasing frequency. This frequency dependent change in slope points to the finite reaction time associated with the domain wall dynamics due to viscous forces. If the rate of change of electric field is very high then the switching takes place over a larger voltage range, while for low applied frequencies, the switching takes place at a lower voltage and over a small voltage range. 


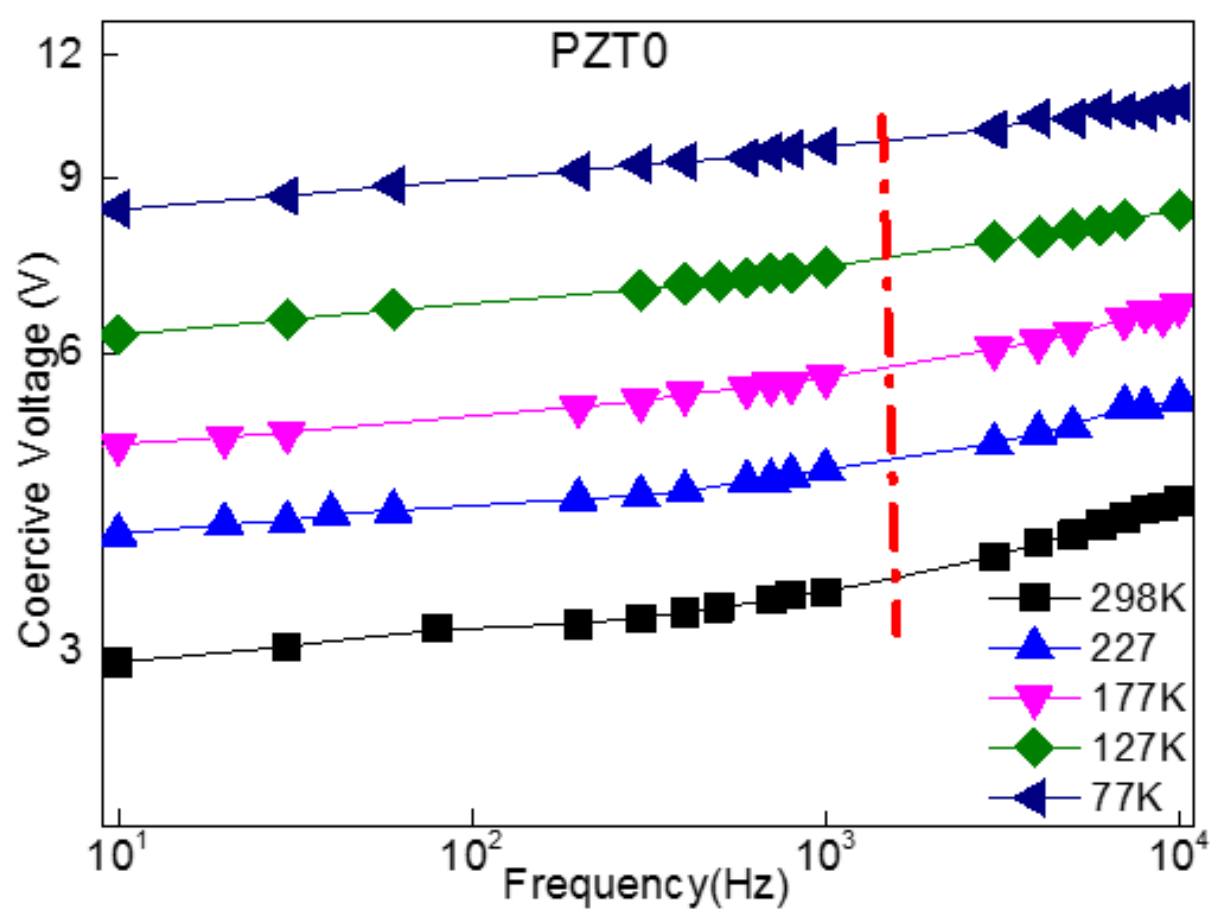

Figure 6.6. The coercive voltage as a function of frequency for different temperatures. The dashed red line denotes the critical frequency where the change in scaling takes place.

In Figure 6.6 we plot the coercive field as a function of frequency for different temperatures on a double log scale. We see that there exist two different linear slopes, which exist even down to $77 \mathrm{~K}$. The difference in the slopes decreases for lower temperature, however the crossover frequency does not change with the temperature. The critical frequency $f_{c r}$ is found to approximately $2 * 10^{3} \mathrm{~Hz}$ by finding the intersection point between the two lines with different slopes representing two different scalings. A similar analysis by Noh ${ }^{16}$ on 001 oriented $\mathrm{PbZr}_{2.2} \mathrm{Ti}_{0.8} \mathrm{O}_{3}$ gave a value of about $500 \mathrm{~Hz}$.

\section{Crossover from no nucleation to TAR}

When the sample is fully switched in one polarization direction then there are no domains in the reverse direction. Hence we should first consider reverse nucleation. Reverse domain nucleation will start for fields above a certain field $E_{N}$, after which the domain nucleation will 
be aided by thermal activation. For higher fields the system enters the TAR regime in which the domain walls can hop over the energy barrier $\Delta E$ in a thermally activated manner with a hopping time constant $\tau \sim \exp (\Delta \mathrm{E} / \mathrm{kT})$ as illustrated in Figure 6.2 (b) (orange line). This energy barrier $\Delta \mathrm{E}$ is dependent on the local pinning energy and the nature and type of the disorder. Because of the finite hopping time constant associated with the hopping the creep motion can only occur when $\tau$ is smaller than $1 / f$, where $f$ is the frequency of the ac field. Hence, for a very high frequency of the ac field the domain wall will not have enough time to follow the electric field to hop over the energy barrier and TAR induced domain wall motion can't take place.

\section{Crossover from TAR to flow regime}

With a further increase of the applied electric field the $\Delta \mathrm{E}$ becomes negligible and the domain wall goes into the viscous flow regime. Since the domain wall velocity in this regime is directly proportional to the applied electric field this difference in domain wall velocities can be used to distinguish TAR from the flow regime as we did in Chapter 4. 

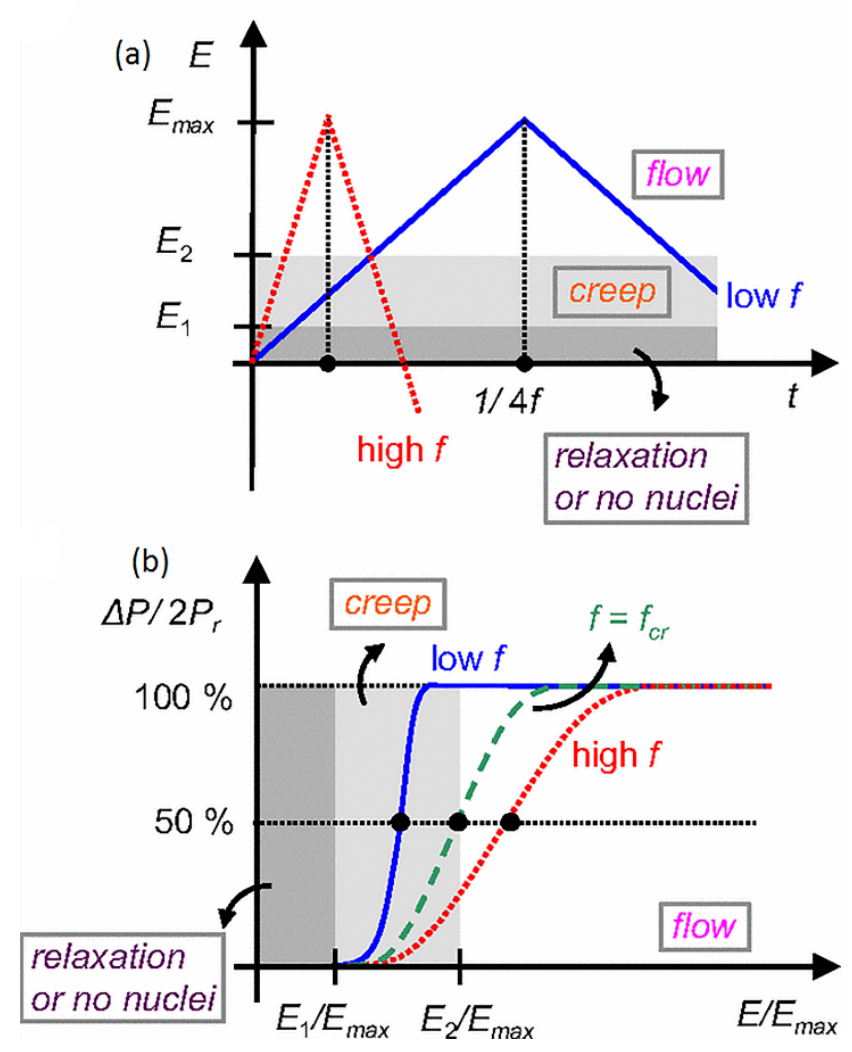

Figure 6.7. (a) Schematic of high frequency and low frequency ac signal and the relative time and magnitude spent in each of the 3 regimes (b) Fractional switching as a function of applied electric field for different sweep rate. The green line denotes the switching at the critical frequency. ${ }^{16}$ (Reproduced with permission)

As mentioned earlier the process of magnetization reversal can be divided into two stages: nucleation of domains with opposite magnetization directions and growth of these domains. Which process dominates depends on system parameters. The relative importance of the above two mechanisms on the switching process depends on the regime in which the system stays longer, which in turn depends on the frequency of the ac field. Figure $6.6(\mathrm{~b})$ shows the fraction of the switched polarization as function of the applied field. It is seen for $E<E 1$ there 
is no nucleation, between E1 and E2 the system is in the TAR regime and beyond that in the viscous flow regime. From Figure 6.6 (a) we can see that, when the field is applied at a very low frequency, the system remains in the TAR regime for a longer time, while if the applied frequency is very high then the system hardly remains in the TAR regime. As can be seen from Figure 6.5 the shape of the loop changes more beyond the critical frequency $\left(2 * 10^{3}\right.$ $\mathrm{Hz}$ ). At a low frequency the system stays in TAR for longer period of time and the switching is dominated by the thermally activated motion. Since the switching happens now in a narrow voltage range (switching can occur at lower voltage due to the longer dwelling time) the loops become sharper with decreasing frequency. As we increase the frequency the system goes to the flow regime very quickly and domain wall flow dominates the switching. Since the time duration at each voltage is very short the switching takes place over a wider voltage range which makes the loop more tilted.

\section{Construction of the T-E phase diagram}

To construct the T-E phase diagram we need to determine the two crossover electric fields. $E_{1}$ is determined by the electric field at which the nucleation starts. We determine the onset of nucleation from the switching current vs voltage as shown in the inset of Figure 6.5. To determine the value of $E_{2}$ we need to consider the frequency dependent hysteresis loops. $E_{2}$ determines the onset of flow dominated switching. We notice that at $f_{c r}$ the switching becomes dominated by domain wall flow. At coercive field $E_{c}$ the sample is $50 \%$ switched. For low frequencies $E_{c}<E_{2}$ and for higher frequencies $E_{c}>E_{2}$, hence we can identify $E_{2}$ with the coercive field value at $f_{c r}$. In Figure 6.7 we plot the T-E phase diagram for the PZT 0 sample. The $E_{N}$ is the nucleation field at $f_{c r}$ and $E_{c}$ is the coercive field at $f_{c r}$. 


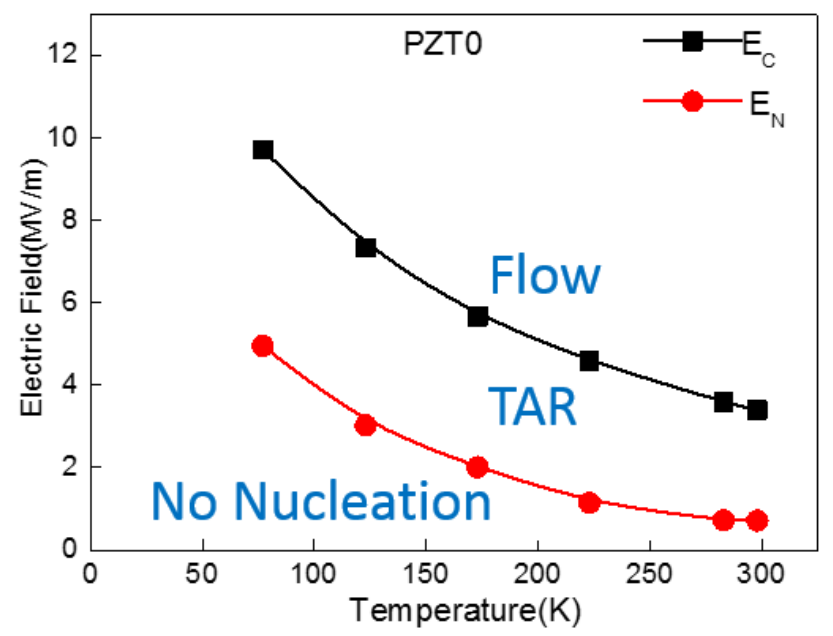

Figure 6.8 Temperature-electric field dynamic phase diagram for PZTO. $E_{N}$ corresponds to the $E_{1}$ and $E_{c}$ corresponds to $E_{2}$ as in Figure 6.3.

\section{Effect of nucleation energy on scaling}

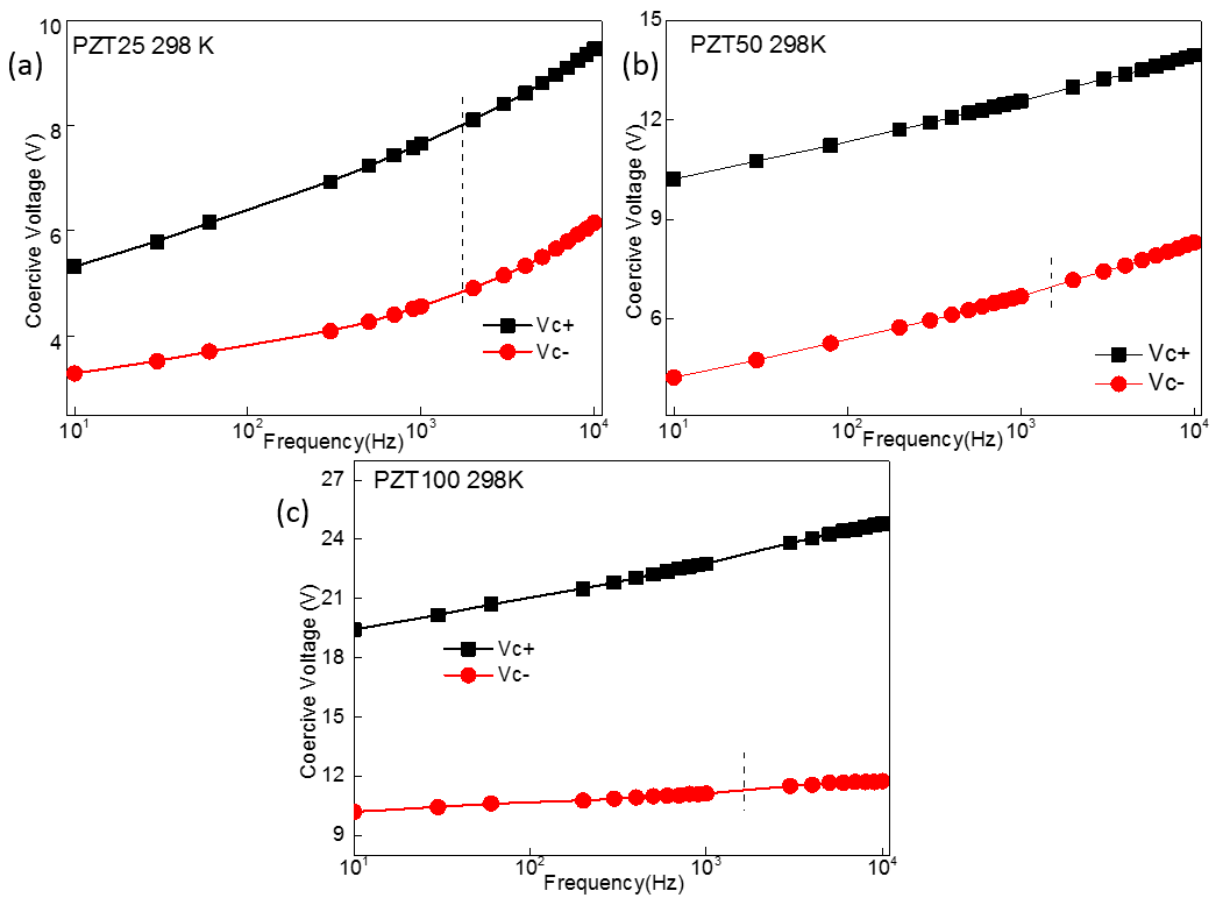

Figure 6. 9 The coercive voltage as a function of frequency for different sam ples at room temperature. The dashed black line denotes the critical frequency where the change in scaling takes place. 
In Figure 6.9 we show the coercive fields as a function of the frequency for the different samples PZT25, PZT50 and PZT100. In Chapter 4 it was discussed that the activation energy increases logarithmically with the ZnO thickness in the WURD direction, but remains almost constant in the WDRU. It is now interesting to see how the scaling is modified by the increase in the nucleation energy in one direction. From the above plots we can see that for the coercive voltage in the negative direction there is a change in slope at about $2 * 10^{3} \mathrm{~Hz}$ (obtained by extrapolating the lines with different slopes), marked with the black dashed line in Figure 6.9 (a) for PZT25. It must be noted here that unlike in Figure 6.6 in figure 6.9 the $Y$ axis was kept in linear scale so that the change in scaling is visible. It clearly shows that for the negative direction there exists two scaling as described above similar as for PZTO. TAR regime dominates at the low frequency and flow governs the switching at higher frequency. This observation is also consistent with our pulse based measurements discussed in Chapter 4 where we observed creep like behaviour at low voltages and flow behaviour at high voltages.

But most interestingly, for the positive coercive field we do not observe any change in frequency scaling for PZT50 and PZT100. Whereas for PZT25 there exists two scaling however the difference in the slopes are much less. This indicates that in the positive direction the films PZT50 and PZT100 switches via only one mechanism. In Chapter 4 we concluded that the film switching in the positive direction is controlled by nucleation only. In case the initial nucleation field becomes much higher than $E_{2}$ (crossover field from TAR to flow) the domain motion switches directly from the nucleation to the flow regime, skipping the intermediate thermally activated regime.

To understand the role of temperature on the switching for different samples we plotted the normalized coercive field values as a function of temperature in Figure 6.10. We can see that the positive coercive field is much less sensitive to temperature change and the higher the coercive field (higher nucleation energy) the less is the temperature sensitivity. This is also reflected in the absence of the TAR for the positive direction. The negative coercive field is much more sensitive to the change in the temperature and has approximately the similar sensitivity with temperature. 

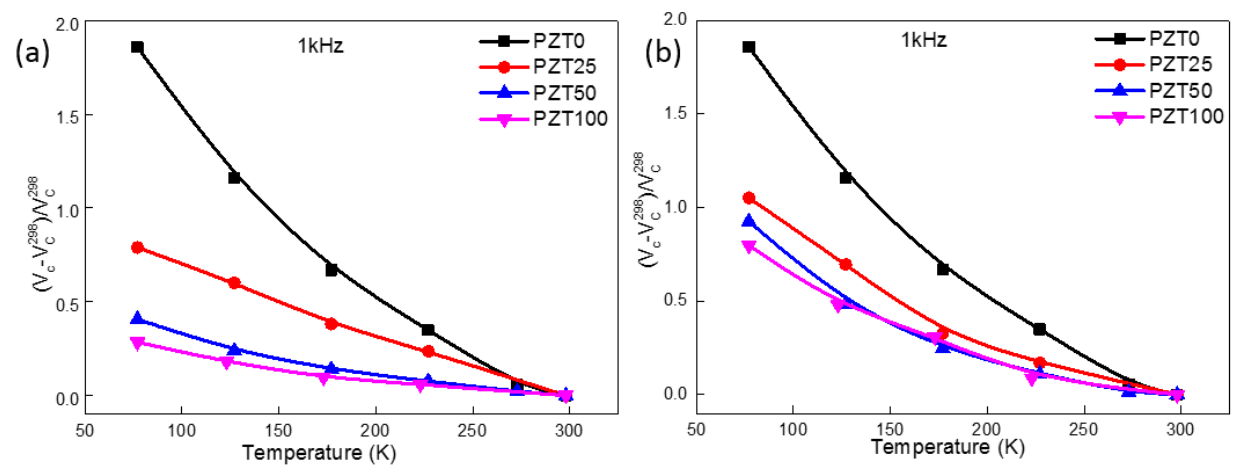

Figure 6.10 Normalized difference of the coercive voltage as a function of temperature for different samples at $1 \mathrm{KHz}$. (a) For the positive direction coercive voltage and (b) for the negative direction coercive voltage

To understand why with increasing coercive field (nucleation energy) the temperature dependence becomes less we need to look at the nucleation mechanism. If $\Delta U_{N}$ is the nucleation barrier then the switching time at a given temperature $T$ can be written $\mathrm{as}^{13,30}$

$$
\tau \sim \exp \left[\left(\Delta E_{N^{-}} P . E\right) / k T\right]
$$

Here $\mathrm{P}$ is the saturation polarization in the activation volume and $\mathrm{E}$ the applied electric field. If $\Delta \mathrm{E}_{\mathrm{N}}$ is higher, then the applied electric field becomes much more important for the switching time compared to the temperature (for an ac signal with a given frequency the switching time needs to be smaller than $1 / f)$. The absence of TAR can have significant effect on the retention and back-switching properties of a ferroelectric thin film. As discussed previously the difference in the $\mathrm{Pr}_{\mathrm{r}}$ and $\mathrm{P}_{\mathrm{s}}$ value in PZTO films is due to a thermally activated motion. Hence if we can increase the nucleation energy this thermally activated backswitching can be minimized.

\subsection{Conclusions}

In conclusion: we have studied the switching mechanism of PZT thin films using frequency and temperature dependent ac dynamics. We observe two different scalings present in the hysteresis loop. We concluded that at low frequency the thermally activated mechanism dominates the switching, whereas at higher frequency the viscous domain wall flow 
mechanism dominates the switching. From the frequency and temperature dependent hysteresis loops study we could construct the T-E phase diagram for the PZTO film and determined the nucleation-TAR $\left(E_{1}\right)$ and TAR-flow $\left(E_{2}\right)$ electrical crossover field lines. We also observed that the thermally activated regime can be suppressed (TAR), when the nucleation energy becomes much higher than $E_{2}$. The suppression of the TAR can lead to better retention and minimize the back-switching in ferroelectric films. 


\subsection{References}

1. T. Tybell, P. Paruch, T. Giamarchi and J. M. Triscone, Physical Review Letters 89 (9) (2002).

2. P. Paruch and J. Guyonnet, Comptes Rendus Physique 14 (8), 667-684 (2013).

3. W. Kleemann, Annual Review of Materials Research 37 (1), 415-448 (2007).

4. T. G. Pascal Chauve, and Pierre Le Doussal, PRB 62 (1) (2000).

5. T. Giamarchi, A. Kolton and A. Rosso, in Jamming, Yielding, and Irreversible Deformation in Condensed Matter (Springer, 2006), pp. 91-108.

6. J. Gorchon, S. Bustingorry, J. Ferre, V. Jeudy, A. B. Kolton and T. Giamarchi, Phys Rev Lett $113(2), 027205$ (2014).

7. S. Jesse, B. J. Rodriguez, S. Choudhury, A. P. Baddorf, I. Vrejoiu, D. Hesse, M. Alexe, E. A. Eliseev, A. N. Morozovska, J. Zhang, L. Q. Chen and S. V. Kalinin, Nature materials 7 (3), 209215 (2008).

8. G. Bertotti and I. D. Mayergoyz, The Science of Hysteresis: Physical modeling, micromagnetics, and magnetization dynamics. (Gulf Professional Publishing, 2006).

9. A. K. Tagantsev, L. E. Cross and J. Fousek, Domains in ferroic crystals and thin films. (Springer, 2010).

10. T. Nattermann, V. Pokrovsky and V. M. Vinokur, Phys Rev Lett 87 (19), 197005 (2001).

11. B. Meyer and D. Vanderbilt, Physical Review B 65 (10) (2002).

12. L. He and D. Vanderbilt, Physical Review B 68 (13) (2003).

13. J. F. Scott, Ferroelectric memories. (Springer Science \& Business Media, 2000).

14. T. A. Moore and J. A. C. Bland, Journal of Physics: Condensed Matter 16 (46), R1369R1386 (2004).

15. Y. J. Shin, B. C. Jeon, S. M. Yang, I. Hwang, M. R. Cho, D. Sando, S. R. Lee, J. G. Yoon and T. W. Noh, Scientific reports 5, 10485 (2015).

16. S. M. Yang, J. Y. Jo, T. H. Kim, J. G. Yoon, T. K. Song, H. N. Lee, Z. Marton, S. Park, Y. Jo and T. W. Noh, Physical Review B 82 (17) (2010).

17. R. Waser, U. Böttger and S. Tiedke, Polar oxides: properties, characterization, and imaging. (John Wiley \& Sons, 2006).

18. R. M. Bozorth and D. Ferromagnetism, New York 849 (1951). 
19. J. S. W. Rayleigh, Phil Mag 23 (1887).

20. C. P. Steinmetz, Trans. Am. Inst. Electr. Eng. 3 (1892).

21. M. Rao, H. R. Krishnamurthy and R. Pandit, Physical Review B 42 (1), 856-884 (1990).

22. M. H. L. Jih-Shin Suen, G. Teeter, and J. L. Erskine, Phys. Rev. B 59 (1999).

23. J.-S. S. a. J. L. Erskine, Phys. Rev. Lett. 78 (1997).

24. M. D. O. B. Raquet, M. Goiran , A.R. Ferta, J.P. Redoules, R. Mamy, and J.C. Ousset, A. Sdaq , A. Khmou Journal of Magnetism and Magnetic Materials 150 (1995).

25. R. M. B. Raquet, and J. C. Ousset, Phys. Rev. B 54 (1996).

26. S. Hashimoto, H. Orihara and Y. Ishibashi, Journal of the Physical Society of Japan 63 (4), 1601-1610 (1994).

27. H. Orihara, S. Hashimoto and Y. Ishibashi, Journal of the Physical Society of Japan 63 (3), 1031-1035 (1994).

28. X. Du and I.-W. Chen, presented at the MRS Proceedings, 1997 (unpublished).

29. D. J. Jung, M. Dawber, J. F. Scott, L. J. Sinnamon and J. M. Gregg, Integrated Ferroelectric S 48 (1), 59-68 (2002).

30. T. N. Igor F. Lyuksyutov, and Valery Pokrovsky, PRB 59 (1999). 


\section{summary}

Modern computing based on Von Neumann architecture and storage devices are based on detecting a change in the state of a material. Hence, bistable materials e.g. ferromagnets and ferroelectrics become a natural choice to achieve this objective in real devices. Since these materials possess two stable states which can be switched from one to another and are also non-volatile in nature they can be used both as a memory element and for non-volatile RAM. However, with the demand for realizing brain inspired computing driven by applications beyond what is achievable by conventional computers e.g. pattern recognition etc. novel material properties need to be explored. Human brain unlike computing devices are analog in nature and dynamically processes data. Hence analog computers in principle can overcome the limitations of digital computers. In order to realize brain like computation in real devices one would need to create multiple switchable non-volatile states. Switching in ferroelectric thin films takes place via heterogeneous nucleation (at the ferroelectricelectrode interface) and subsequent growth of domains. In this thesis it is shown how we can create multiple states in a capacitor structure that comprises ferroelectric PZT by controlling the switching through the manipulating the statistics of the nucleation energy. We achieved this by controlling the local electric field at the ferroelectric-electrode interface by coupling the switchable polarization of PZT and the non-switchable polarization of ZnO.

In Chapter 2 the growth and characterization of our model system is discussed. Epitaxial thin film heterostructures of SrRuO3 $(80 \mathrm{~nm}) / \mathrm{PbZr}_{0.58 \mathrm{Ti}_{0.42} \mathrm{O}_{3}}(1000 \mathrm{~nm})$ (PZT)/ZnO (25-150 $\mathrm{nm}) / \mathrm{SrRuO} 3(80 \mathrm{~nm})$ were fabricated on $\mathrm{SrTiO}_{3}$ (111) substrates using pulsed laser deposition. These heterostructures were utilized to manipulate the polarization switching dynamics of PZT through polarization coupling. By being able to grow $\mathrm{ZnO}$ of different thicknesses with similar a rms roughness allowed us to manipulate the disorder-(induced by the roughness)-to-order ratio. This relative disorder value was utilized later in the thesis to manipulate the overall switching statistics.

In Chapter 3 discusses how the electric field at the ferroelectric-electrode interface can be manipulated by controlling the thickness of the non-switchable $\mathrm{ZnO}$ layer. Our results have 
shown that the imprint or the offset voltage is directly proportional to the thickness of the ZnO layer. It has also been demonstrated that the ferroelectric imprint remains stable with the electric field cycling and electric field stress assisted aging.

In Chapter 4 the switching dynamics of the aforementioned heterostructures have been analysed using the KAl (Kolmogorov Avrami Ishibashi) and NLS (nucleation limited switching) models. It was concluded that the nucleation activation energy increased exponentially with the built-in electric field at the ferroelectric-electrode interface. The extreme sensitivity of the local switching times on the presence of fixed dipoles near the surface enables us to create independent regions within the same capacitor structure each having individual switching times. These independent regions were formed by controlling the relative roughness of the fixed dipole layer. By enabling the ferroelectric to switch in a multivaluedmanner, an important step toward a neuron like adaptive memory and multi-state memories was achieved.

In Chapter 5 the semiconducting nature of the ferroelectric PZT was investigated. Temperature dependent leakage measurements (I-V) were performed on $\mathrm{SRO} / \mathrm{PZTO} / \mathrm{ZnO} / \mathrm{SRO}$ heterostructures to understand the leakage mechanism acting in these heterostructures. The leakage mechanism was found to be due to interface controlled Schottky emission and was analysed using the Schottky-Simmons relation. This model assumes that the interface controls the charge injection while the bulk controls the transport. Temperature dependent capacitance voltage (C-V) measurements was used to determine the free charge carriers inside the PZT thin films. The interface trap density was determined using the parallel conductance method and was found to be of the order of $10^{10}$ $\mathrm{cm}^{-2}$. It was also demonstrated that the capacitive contributions are much higher than the resistive leakage contribution.

In Chapter 6 the dynamic switching behaviour of the aforementioned heterostructures was analyzed in terms of the motion of an elastic media in a disordered potential. The dynamic coercive fields of PZT and PZT-ZnO heterostructures introduced in previous chapters were measured as a function of the frequency $\left(10-10^{4} \mathrm{~Hz}\right)$ and temperature (77-298 K). Two scaling regimes in the $\log \left(E_{c}\right)$ versus log (frequency) plot was observed for the PZTO sample, 
which existed over the whole temperature range. The two type of scaling arise from the dynamic cross over from the thermally activated to flow regime of the ferroelectric domain wall motion. A dynamic temperature $(T)$ - electric field (E) phase diagram of the ferroelectric switching dynamics for PZT0 sample was also constructed. For the PZT 25, PZT50 and PZT100 samples only one frequency scaling was observed for the positive coercive field direction whereas in the negative direction two different types of scaling similar to that of PZTO were observed. The coercive field in the positive direction was much less sensitive to temperature unlike the coercive field in the negative direction. 


\section{samenvatting}

Moderne gegevensverwerkingstechnieken zijn gebaseerd op de Von Neumann architectuur en opslagapparaten zijn gebaseerd op het detecteren van de verandering van een toestand in het materiaal. Daarom zijn bistabiele materialen zoals ferromagneten en ferroelektrisch een logische keus om deze effecten te realiseren in apparaten. Deze materialen hebben twee stabiele toestanden die geschakeld kunnen worden van een toestand naar een andere. Bovendien is er geen spanning vereist om een toestand aan te houden. Hierdoor kunnen ze gebruikt worden als geheugen en niet-vluchtig RAM. Echter met de vraag naar het realiseren van op hersenen gebaseerde gegevensverwerkingstechnieken, die gedreven worden door applicaties die verder gaan dan conventionele computers zoals patroon herkenning etc. moeten er nieuwe materiaal eigenschappen onderzocht worden.

Het menselijke brein van is nature een analoog dataverwerkingsmechanisme en verwerkt data dynamisch, tegenstelling tot computers. Analoge rekenmethodes zouden in principe de limitaties van digitale computers moeten overwinnen. Om hersen achtige berekeningen te realiseren in apparaten zijn er meerdere schakelbare niet vluchtig toestanden nodig. Het schakelen van toestanden in ferroelektrische dunne films gaat via heterogene nucleatie op het ferroelektrische electrode interface en de daaropvolgende groei van de domeinen.

Dit proefschrift beschrijft hoe er meerdere toestanden in een condensator structuur gemaakt worden. De structuur bestaat uit ferroelektrisch PZT dat kan schakelen door middel van manipulatie van de statistiek van de nucleatie energy. Dit is bereikt door het controleren van he lokale elektrische veld op het ferroelektrische electrode interface door de koppeling van de schakelbare polarisatie van het PZT en de non-schakelbare polarisatie van ZnO.

In hoofdstuk 2 wordt de groei en karakterisatie van het model systeem besproken. Epitaxiale dunne film heterostructuren van SrRuO3 $(80 \mathrm{~nm}) / \mathrm{PbZr}_{0.58} \mathrm{Ti}_{0.42} \mathrm{O}_{3}(1000 \mathrm{~nm})(\mathrm{PZT}) / \mathrm{ZnO}(25-$ $150 \mathrm{~nm}) / \mathrm{SrRuO} 3(80 \mathrm{~nm})$ zijn gemaakt op $\mathrm{SrTiO}_{3}(111)$ substraten met behulp van gepulste laser depositie. Deze heterostructuren zijn gebruikt om de gepolariseerde schakel dynamiek van PZT te manipuleren door middel van polarisatie koppeling. De mogelijkheid om ZnO te 
groeien met verschillende diktes met een vergelijkbare ruwheid heeft ons toegelaten om de wanorder (geïnduceerd door de ruwheid) naar order ratio te manipuleren. De relatieve wanorder waarde wordt later gebruikt in dit proefschrift om de totale schakel statistiek te manipuleren.

In hoofdstuk 3 beschrijft hoe een elektrisch veld het ferroelektrische electrode interface kan manipuleren door middel van de dikte van de niet schakelbare ZnO laag te controleren. De resultaten hebben laten zien dat de afdruk of de offset spanning direct gerelateerd zijn aan de dikte van de $\mathrm{ZnO}$ laag. Ook wordt hier beschreven dat de ferroelektrische afdruk stabiel blijft met het schakelen van het elektrisch veld en elektrisch veld stres gesteunde veroudering.

In hoofdstuk 4 is de schakel dynamica van de bovengenoemde heterostructuur geanalyseerd met behulp van de KAl (Kolmogorov Avrami Ishibashi) en NLS (nucleation limited switching) modellen. Met de conclusie dat de nucleatie activatie energy exponentieel toeneemt met het ingebouwde elektrische veld op het ferroelektrische electrode interface. De extreme gevoeligheid van de lokale schakeltijden op de aanwezigheid van vaste dipolen in de buurt van het oppervlakte geeft de mogelijkheid om onafhankelijke gebieden te maken binnen dezelfde condensator structuur die ieder een eigen schakeltijd hebben. Deze onafhankelijke gebieden zijn gevormd door het controleren van de relatieve ruwheiden van de vaste dipool laag. Door te zorgen dat het ferroelektrische materiaal op meerdere manieren geschakeld is, is een belangrijke stap in de richting van neuronen achtige adaptief geheugen en geheugen met meerdere toestanden.

In hoofdstuk 5 zijn de halfgeleidende eigenschappen van ferroelektrisch PZT onderzocht. De temperatuurafhankelijke lekstroom metingen (I-V) zijn uitgevoerd op SRO/PZTO/ZnO/SRO heterostructuren. Dit is om het lekstroom mechanisme te begrijpen die werkt op deze heterostructuren. Het lekstroom mechanisme dat gevonden werd, werd gecontroleerd door de interface gecontroleerde Schottky emissie en was geanalyseerd door de SchottkySimmons relatie. Dit model gebruikt de aannamen dat het interface de ladingsinjectie regelt, terwijl de bulk de transport regelt. De temperatuurafhankelijke capaciteit- en 
spanningsmetingen (C-V) zijn gebruikt om de vrije ladingsdragers in de PZT films te bepalen. De interface valdichtheid was bepaald door middel van de parallelle geleidingsmethode en deze was in de order van of $10^{10} \mathrm{~cm}^{-2}$. Ook laat dit hoofdstuk zien dat de capacitieve bijdrages veel groter zijn dan de weerstandslekstroom bijdrage.

In hoofdstuk 6 is het dynamische schakelgedrag van de bovengenoemde heterostructuren geanalyseerd, met betrekking tot de beweging van het elastisch media in een wanordelijk potentiaal. De dynamische coërcieve velden van PZT en PZT-ZnO heterostructuren, die geïntroduceerd zijn in de vorige hoofdtukken zijn gemeten als functie van frequentie (10 $10^{4} \mathrm{~Hz}$ ) en temperatuur (77-298 K). Twee schalingsregimes in de logaritmische $\left(E_{c}\right)$ tegen de logaritmische (frequentie) grafiek zijn geobserveerd voor het PZTO monster, over het gehele temperatuurregime. De twee type schalingen komen door de dynamische cross-over van de thermische geactiveerde naar de flow regime van de ferro-elektrische domein muur beweging. Een dynamisch temperatuur (T) - elektrisch veld (E) fase diagram van de ferroelektrische schakel dynamiek voor het PZTO monster is ook gemaakt.

Voor de PZT 25, PZT50 en PZT100 monsters is er maar één frequentie schaling geobserveerd voor de positieve coërcief veld richting. De negatieve richting zijn er twee verschillende typen van schaling geobserveerd. Dit is vergelijkbaar met het PZTO monster. Het coërcief veld in de positieve richting is veel minder gevoelig voor temperaturen in tegenstelling tot het coërcief veld in de negatieve richting. 


\section{Acknowledgements}

At the conclusion I come to the most gratifying and humbling part of writing the thesis. With the very limited knowledge of the language I dare to try express my gratitude to all my wellwishers, without whose support and encouragement this thesis would probably not have come to fruition. As Alexander Bell had aptly put "Great discoveries and improvements invariably involve the cooperation of many minds. I may be given credit for having blazed the trail, but when I look at the subsequent developments, I feel the credit is due to others rather than to myself." truly reflects my emotions at this juncture.

At first I would like to express my heartfelt gratitude to my supervisors Prof. Guus Rijnders and Prof. Gertjan Koster for giving me the opportunity to pursue my PhD at IMS. I am indeed grateful to you for having the confidence in me and giving me a free hand to pursue my research independently. I am very much thankful to you for your understanding and support in difficult times and providing all the required research facilities. Gertjan, I was indeed fortunate to have an understanding supervisor like you, I am ever so grateful for your guidance and all that I learnt from you. Thank you for tolerating all my idiosyncrasies, it is really commendable.

I would also like to take this opportunity to express my gratitude to my M.Sc supervisors at I.I.Sc Bangalore, Prof. C. N. R. Rao and Prof. U. V. Waghmare for all their support and help. Mere words can't express how thankful I am for your support during the most difficult part of my life, whatever little I could achieve is because of the best possible education I received during my masters. Sir, you have always been my source of inspiration and motivation.

I would also like to thank the organizers of ISOE and IEEE-ISAF for giving a very nice platform for exchanging ideas and giving the opportunity to interact with the leading experts in the field. I would also like to express my appreciation for scihub for breaking the man made barriers impeding dissemination of science.

I would like to acknowledge the support and technical expertise of Dominic, Henk and Frank for their wonderful job and the training, you guys are the real backbone. I would also like to 
thank Marion, Josee and Cecile for all your official and onboarding help. Marion you have always been super-efficient.

I again wanted to be mean but for a change I will not, so I would indeed like to thank my colleagues and friends at IMS. It was indeed a pleasure working with you guys and all your help and support and making IMS a fun place. Thanks to my officemates Jose, Xin, Alim, David and Laura for tolerating me in your office and for the nice chit chat. I am indeed thankful to all the IMSers both past and present Kenan, Kurt, Alim, Werner, Minh, Tom, Pramodh, Brian, Rik, Pablo, Bouwe, Jun, Ron, Jaap, Petra, Daniel, Ruud, Thanh, Gerard, Sjoerd, Huiyu, Ben, Deepak, Minh, Tjeerd, Zhaoliang, Lin, Nirupam Maarten, Karen, Wouter, Rogier, Michelle, Roy and Suresh for all your help and the fun provided. Thanks Tom for translating the summary, and being one of the IMS drammers along with Werner for all the dram. Thanks Kurt for the little exercises for my grey cells. It was indeed a pleasure to work and discuss with you Evert, Mark and Andre. I would indeed like to thank Anuj and Debakanta Ji for all their guidance and help in the initial days, it was really invaluable.

Life would have been completely dull and boring if not for you friends. Himanshu thanks. Sahana thanks for not yet charging any money for listening to you, hopefully the free days for the audiences will be over soon. Harshita and Kartikeya for all the nice food good times. Mitava \& Naveen for feeding me with so much good food with shameless reciprocity from my side, sometimes Newton also fails I guess. I would indeed like to express my gratitude to the AADHAAR fraternity, guys you are indeed doing a great job lease keep it up. I would also like to express my thanks to Hemant, Deva, Vishal Aggarwal, Vishal Metri, Ravi, Manas, Shantanu, Poorvi, Nikos, Sourish, Rana ji and Ankur. I am indeed very much grateful to Pramod and Vishakha ji for hosting so many times and all the good times.

I would really like to extend my thanks to my "Swiss"friends Alberto and Tomas, for all the help and friendship when I first left India six years ago.

I would also like to thank my paranymphs Himanshu and Werner for all the help and support. I am also thankful to my friends and seniors from I.I.Sc for all your support over the years Tamoghna, Barun, Saurav, Ankur, Soupi, Kanishka da, and Claudy. 
My friends from my innocent years Shilpak, Rakesh, Raja, Samarjit and Avick big thanks to you guys.

Last but not the least my family Baba, Maa and Bhai this thesis belongs to you, nothing less. Without your unconditional love and support I couldn't have done anything let alone a PhD thesis. I don't have any words to express my gratitude.

\section{Anirban Ghosh}

Enschede, September 2016 

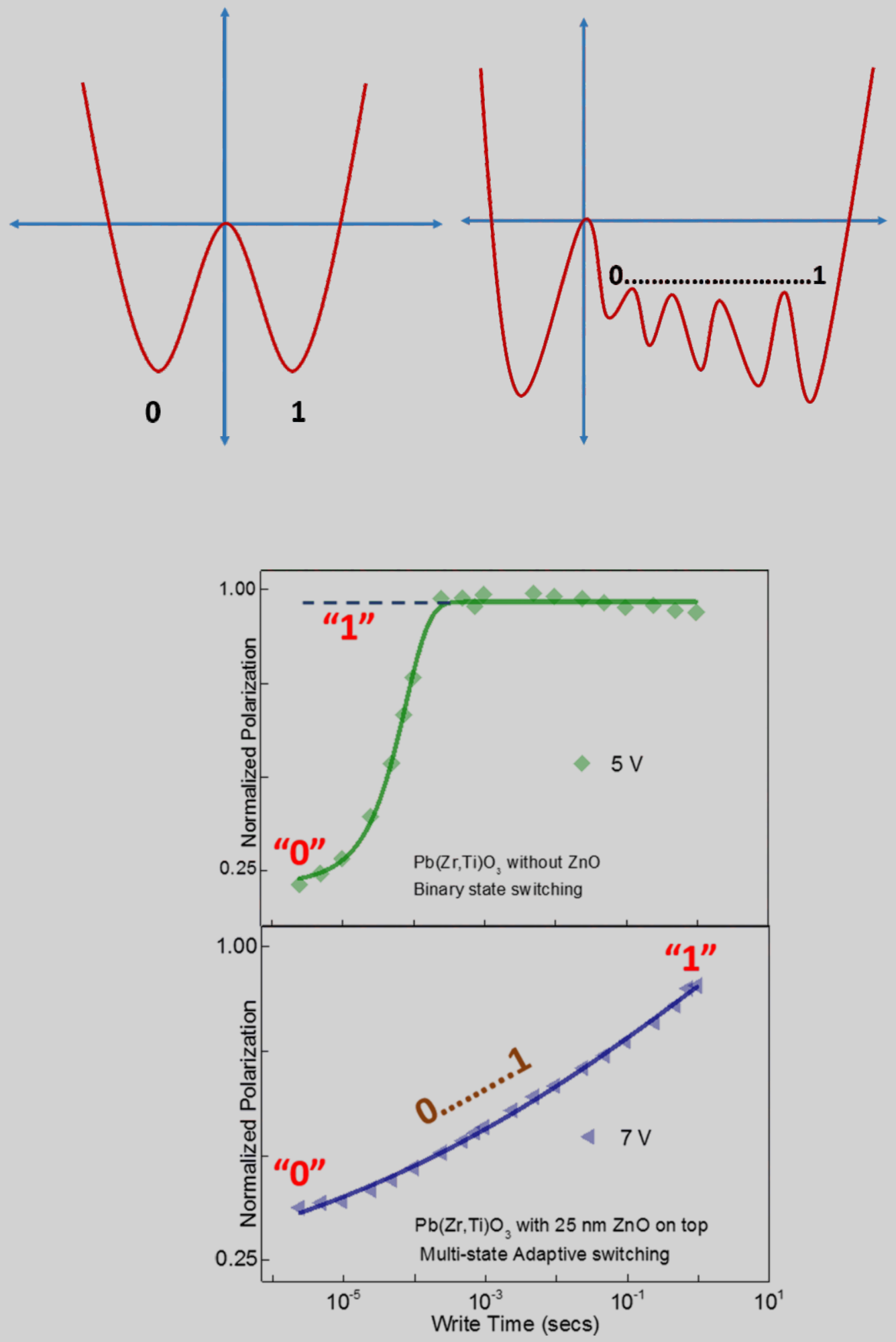

ISBN: 978-90-365-4196-1 